\title{
Chemoselective Cobalt(I)-Catalyzed Cyclotrimerization of (Un)Symmetrical 1,3- Butadiynes for the Synthesis of 1,2,4-Regioisomers
}

Sebastian M. Weber and Gerhard Hilt*

\section{SUPPORTING INFORMATION}

\section{Table of Content}

General Information

Possible Chemo- and Regioisomers of [2+2+2] Cycloaddition Products Starting from S2 Unsymmetrical 1,4-Disubstituted 1,3-Butadiynes

Optimization of the Reaction Conditions for the Cyclotrimerization of Unsymmetrical S3 1,3-Butadiynes

Confirmation of the Optimized Reaction Conditions for the Cyclotrimerization of Symmetrical S5

1,3-Butadiynes

Synthesis of Unsymmetrical substituted 1,3-Butadiynes 


\section{General Information}

All reactions with water- and/or air-sensitive starting materials were carried out in pre-dried glass wares under Argon atmosphere utilizing standard Schlenk techniques. All used solvents were dried over molecular sieves (3 $\AA$ ) and they were degassed prior to use. Thin layer chromatography (TLC) was carried out on prefabricated plates (silica gel 60, F254 with fluorescence indicator) by Macherey Nagel. Column Chromatography was carried out on silica gel 60 (40-63 um, 230-400 mesh) by Macherey Nagel. Commercially available chemicals were used without further purification. Non-commercial reagents were synthesized by literature-known procedures. The cobaltbased precatalyst $\mathrm{CoBr}_{2}$ (dppe) was prepared following a literature-known procedure. ${ }^{1}$ Zinc iodide was dried at $200^{\circ} \mathrm{C}$ in vacuo and stored under Argon atmosphere.

${ }^{1} \mathrm{H}$ and ${ }^{13} \mathrm{C} \mathrm{NMR}$ were either recorded on a Bruker Fourier $300 \mathrm{HD}$ or a Bruker Avance III 500HD spectrometer at room temperature utilizing preset pulse programs. The chemical shifts are given in parts per million (ppm). The residual solvent signal (CDCl $3:{ }^{1} \mathrm{H}$ NMR: 7.26 ppm, ${ }^{13} \mathrm{C}$ NMR: $77.16 \mathrm{ppm}$, DMSO-d6: ${ }^{1} \mathrm{H}$ NMR: $2.50 \mathrm{ppm}$, ${ }^{13} \mathrm{C} \mathrm{NMR}: 39.52 \mathrm{ppm}$ ) was used for calibration referred to tetramethylsilane. ${ }^{19} \mathrm{~F}$ NMR were recorded on a Bruker Avance III 500HD spectrometer at room temperature utilizing preset pulse programs. The chemical shifts are given in ppm. Hexafluorobenzene ( ${ }^{19} \mathrm{~F}$ NMR: $\left.-164.9 \mathrm{ppm}\right)$ or fluorobenzene $\left({ }^{19} \mathrm{~F} \mathrm{NMR:}-113.8 \mathrm{ppm}\right)$ was used as an internal standard. Infrared spectra were recorded on a Bruker Tensor 27 FT-IR spectrometer. The absorption bands are given in wave numbers $\left(\mathrm{cm}^{-1}\right)$. High resolution mass spectra (EI) were recorded on a Thermo Scientific DFS spectrometer. The ionization was accomplished by electron ionization (EI) at an energy of $70 \mathrm{eV}$.

GC/ MS spectra were recorded on a Shimadzu GC 2010 Plus gas chromatograph coupled with a Shimadzu QP2020 mass detector.

Melting points were measured on a Gallenkamp MPD 350 BM 2.5 device. All specified melting points are not corrected. 
Possible Chemo- and Regioisomers of $[2+2+2]$ Cycloaddition Products Starting from Unsymmetrical 1,4-Disubstituted 1,3-Butadiynes.
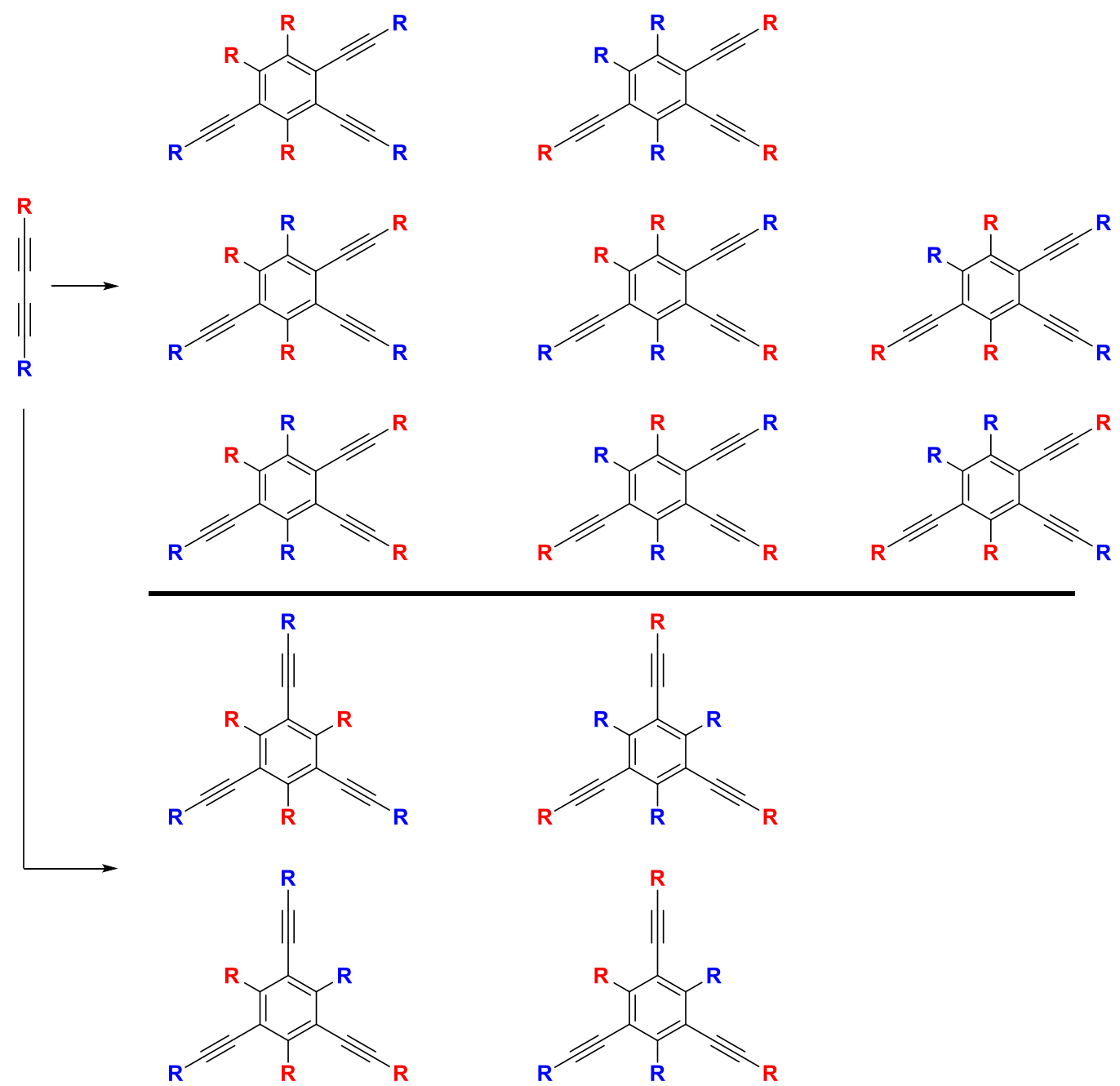


\section{Optimization of the Reaction Conditions for the Cyclotrimerization of Unsymmetrical}

\section{1,3-Butadiynes}

\section{General Procedure 1:}

Under argon atmosphere the corresponding metal salt (5-10 mol \%), ligand (5-10 mol \%), zinc dust (10-20 mol \%) and zinc iodide (10-20 mol \%) were added to a pre-dried reaction vessel. The solids were dried for $15 \mathrm{~min}$ in vacuo. After that the catalyst system was suspended in a dry solvent $(0.3 \mathrm{~mL})$ and it was stirred for $15 \mathrm{~min}$ at ambient temperature. Next, the 1,3-butadiyne $(0.3 \mathrm{mmol}, 1.00$ equiv.) was added and the reaction mixture was stirred for 4-18 $\mathrm{h}$ at the below mentioned temperature (room temperature, up to $60^{\circ} \mathrm{C}$ ). Then fluorobenzene ( $0.30 \mathrm{mmol}, 1.00$ equiv.) was added and a small amount of the mixture was filtered over a short plug of silica gel into a NMR tube. The conversion and the ratio of regioisomers were detected by ${ }^{19} \mathrm{~F}$ NMR spectroscopy.

Table S1: Optimization of the reaction conditions for the cyclotrimerization of unsymmetrical substituted 1,3-butadiyne.
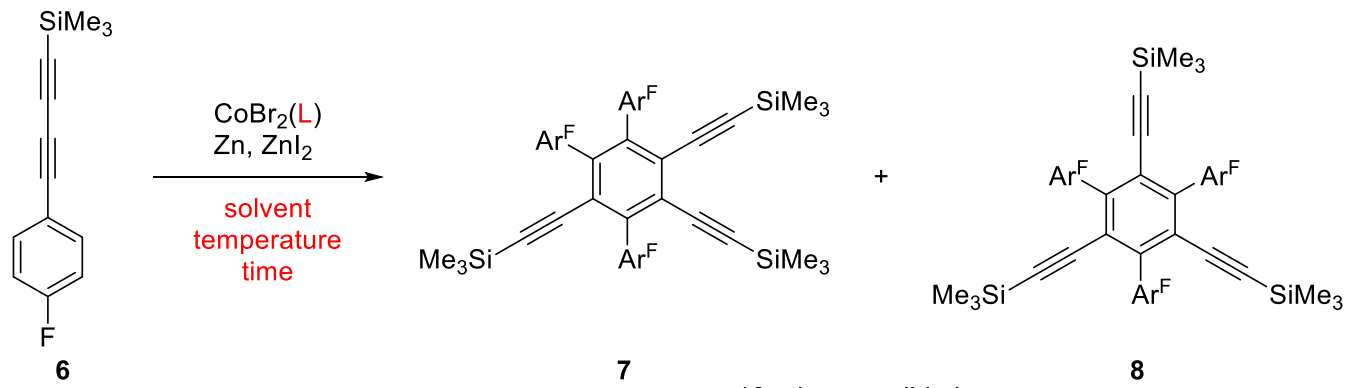

+10 other possible isomers

8

\begin{tabular}{|c|c|c|c|c|c|c|c|}
\hline no. & metal salt & ligand & solvent & $\mathrm{T}\left({ }^{\circ} \mathrm{C}\right)$ & time (h) & conversion $(\%)^{a}$ & ratio $7: 8$ \\
\hline 1 & $\mathrm{CoBr}_{2}$ & none & $\mathrm{MeCN}$ & $\mathrm{rt}$ & $18 \mathrm{~h}$ & $>99$ & $69: 31$ \\
\hline 2 & $\mathrm{CoBr}_{2}$ & $\mathrm{Cy}_{2}$ (diimine) & $\mathrm{MeCN}$ & $\mathrm{rt}$ & $18 \mathrm{~h}$ & 90 & $63: 37$ \\
\hline 3 & $\mathrm{CoBr}_{2}$ & py-imine & $\mathrm{MeCN}$ & $\mathrm{rt}$ & $18 \mathrm{~h}$ & 86 & $65: 35$ \\
\hline 4 & $\mathrm{CoBr}_{2}$ & $i \mathrm{Pr}_{2}$ diimine & $\mathrm{MeCN}$ & $\mathrm{rt}$ & $18 \mathrm{~h}$ & 0 & 0 \\
\hline 5 & $\mathrm{CoBr}_{2}{ }^{\mathrm{b}}$ & $\left(\mathrm{PPh}_{3}\right)_{2}$ & $\mathrm{MeCN}$ & $\mathrm{rt}$ & $18 \mathrm{~h}$ & 44 & $85: 15$ \\
\hline 6 & $\mathrm{CoBr}_{2}$ & dppe & $\mathrm{MeCN}$ & $\mathrm{rt}$ & $18 \mathrm{~h}$ & 0 & 0 \\
\hline 7 & $\mathrm{CoBr}_{2}$ & $\mathrm{Cy}_{2}$ (diimine) & DCM & $\mathrm{rt}$ & $18 \mathrm{~h}$ & 0 & 0 \\
\hline 8 & $\mathrm{CoBr}_{2}{ }^{\mathrm{b}}$ & bpy & $\mathrm{MeCN}$ & $\mathrm{rt}$ & $18 \mathrm{~h}$ & $>99$ & $71: 29$ \\
\hline 9 & $\mathrm{CoBr}_{2}{ }^{\mathrm{b}}$ & phenMe 2 & $\mathrm{MeCN}$ & $\mathrm{rt}$ & $18 \mathrm{~h}$ & $>99$ & $68: 32$ \\
\hline 10 & $\mathrm{CoBr}_{2}$ & $\mathrm{Cy}_{2}$ (diimine) & THF & 60 & $72 \mathrm{~h}$ & $>99$ & $64: 36$ \\
\hline 11 & $\mathrm{CoBr}_{2}{ }^{\mathrm{b}}$ & dppm & $\mathrm{MeCN}$ & 60 & $18 \mathrm{~h}$ & 69 & $74: 26$ \\
\hline 12 & $\mathrm{CoBr}_{2}$ & dppe & $\mathrm{CH}_{2} \mathrm{Cl}_{2}$ & 40 & $72 \mathrm{~h}$ & 30 & $>99: 1$ \\
\hline 13 & $\mathrm{CoBr}_{2}$ & dppe & $\mathrm{Et}_{2} \mathrm{O}$ & 40 & $18 \mathrm{~h}$ & 87 & $>99: 1$ \\
\hline 14 & $\mathrm{CoBr}_{2}$ & dppe & Aceton & 60 & $18 \mathrm{~h}$ & $>99$ & $98: 2$ \\
\hline 15 & $\mathrm{CoBr}_{2}$ & dppe & EtOAc & 60 & $18 \mathrm{~h}$ & $>99$ & $97: 3$ \\
\hline 16 & $\mathrm{CoBr}_{2}$ & dppe & $\mathrm{MeCN}$ & 60 & $18 \mathrm{~h}$ & $>99$ & $97: 3$ \\
\hline 17 & $\mathrm{CoBr}_{2}$ & dppe & THF & 60 & $18 \mathrm{~h}$ & $>99$ & $>99: 1$ \\
\hline 18 & $\mathrm{CoBr}_{2}$ & $\mathrm{Cy}_{2}$ (diimine) & THF & 60 & $72 \mathrm{~h}$ & $>99$ & $64: 36$ \\
\hline 19 & $\mathrm{CoBr}_{2}{ }^{\mathrm{b}}$ & disulfide & THF & 60 & $72 \mathrm{~h}$ & 0 & 0 \\
\hline
\end{tabular}




\begin{tabular}{|c|c|c|c|c|c|c|c|}
\hline no. & metal salt & ligand & solvent & $\mathrm{T}\left({ }^{\circ} \mathrm{C}\right)$ & time (h) & conversion (\%) & ratio $7: 8$ \\
\hline 20 & $\mathrm{CoBr}_{2}$ & dppe & Toluol & 60 & $18 \mathrm{~h}$ & 0 & 0 \\
\hline 21 & $\mathrm{Co}(\mathrm{acac}){ }_{2}^{\mathrm{c}}$ & - & THF & 60 & $18 \mathrm{~h}$ & 0 & 0 \\
\hline 22 & $\mathrm{NiBr}_{2}{ }^{\mathrm{d}}$ & dppp & THF & 60 & $18 \mathrm{~h}$ & $>99$ & $>99: 1^{f}$ \\
\hline 23 & $\mathrm{NiBr}_{2}{ }^{\mathrm{e}}$ & $\mathrm{Cy}_{2}$ (diimine) & $\mathrm{MeCN}$ & -30 & $4 \mathrm{~h}$ & 0 & 0 \\
\hline 24 & $\mathrm{CoBr}_{2}$ & $\mathrm{Cy}_{2}$ (diimine) & $\mathrm{MeCN}$ & -40 & $18 \mathrm{~h}$ & 62 & $60: 40$ \\
\hline 25 & $\mathrm{CoBr}_{2}$ & $\mathrm{Cy}_{2}$ (diimine) & $\mathrm{MeCN}$ & -78 & $18 \mathrm{~h}$ & $<10$ & --- \\
\hline 26 & $\mathrm{CoBr}_{2}{ }^{\mathrm{b}}$ & bpy & MeCN & $\mathrm{rt}$ & $18 \mathrm{~h}$ & $>99$ & $71: 29$ \\
\hline 27 & $\mathrm{CoBr}_{2}{ }^{\mathrm{b}}$ & BINAP & $\mathrm{MeCN}$ & $\mathrm{rt}$ & $18 \mathrm{~h}$ & $>99$ & $85: 15^{g}$ \\
\hline 28 & $\mathrm{CoBr}_{2}{ }^{\mathrm{b}}$ & $\mathrm{P}(\mathrm{OAr})_{3}$ & $\mathrm{MeCN}$ & $\mathrm{rt}$ & $18 \mathrm{~h}$ & $>99$ & $70: 30$ \\
\hline
\end{tabular}

a) Reaction conditions: metal salt (5 mol \%), Zn, $\mathrm{ZnI}_{2}$ (10 mol \% each), 1,3-butadiyne 6 (0.30 mmol, 1.0 equiv.), solvent $(0.3 \mathrm{~mL})$. Conversion and ratio were determined by ${ }^{19} \mathrm{~F}$ NMR spectroscopy with fluorobenzene as internal standard. $\mathrm{Cy}_{2}$ (diimine): dicyclohexylethane-1,2-diimine; py-imine: $N$-mesityl-1-(pyridin-2-yl)methanimine; $i \mathrm{Pr}_{2}$ diimine: $1,1^{\prime}$-(pyridine2,6-diyl)bis(N-(2,6-diisopropylphenyl)ethan-1-imine); bpy: 2,2'-bipyridine; phenMe $\mathrm{e}_{2}$ : 2,9-dimethyl-1,10-phenanthroline; dppm: 1,1-bis(diphenylphosphino)methane; dppe: 1,2-bis(diphenylphosphino)ethane; BINAP: 2,2'-bis(diphenylphosphino)1,1'-binaphthyl; disulfide: 1,2-bis(4-methoxyphenyl)thio)ethane, $\mathrm{P}(\mathrm{OAr})_{3}$ : tris(2,4-di-tert-butylphenyl)phosphite. b) Different reaction conditions in comparison to a): cobalt salt (10 mol \%), ligand (10-20 mol \%) Zn, Znl 2 (20 mol \% each). c) $10 \mathrm{~mol} \%$ of $\mathrm{Co}(\mathrm{acac})_{2}$ was used as metal source instead of cobalt catalyst. acac: acetylacetonate. d) $\mathrm{NiBr}_{2}$ (dppe) (5 mol \%) was used instead of cobalt catalyst. e) $\mathrm{NiBr}_{2}(10 \mathrm{~mol} \%)$, ligand (10 mol \%) was used. f) one side product was observed in a slight amount. g) many side reactions were observed. 


\section{Confirmation of the Optimized Reaction Conditions for the Cyclotrimerization of}

\section{Symmetrical 1,3-Butadiynes}

All optimization reactions were carried out as described above according to the general procedure 1.

Table S2: Optimization of the reaction conditions for the cyclotrimerization of symmetrical substituted 1,3-butadiynes.
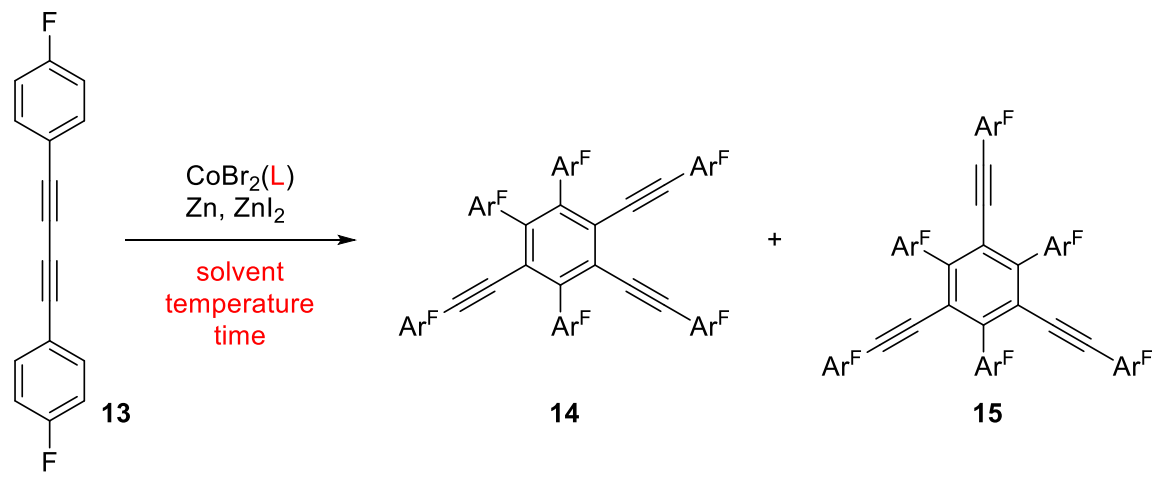

14

15

\begin{tabular}{|c|c|c|c|c|c|c|c|}
\hline no. & cobalt catalys & solvent & subst. conc. (M) & $\mathrm{T}\left({ }^{\circ} \mathrm{C}\right)$ & Time (h) & conversion $(\%)^{a}$ & ratio 7:8 \\
\hline 1 & $\mathrm{CoBr}_{2}$ (pyimine) & $\mathrm{MeCN}$ & 0.1 & 0 to rt & 18 & 0 & - \\
\hline 2 & $\mathrm{CoBr}_{2}$ (pyimine) & $\mathrm{CH}_{2} \mathrm{Cl}_{2}$ & 1.0 & 0 & 1 & 0 & - \\
\hline 3 & $\mathrm{CoBr}_{2}\left(\mathrm{Cy}_{2}\right.$ diimine $)$ & THF & 1.0 & 0 to $\mathrm{rt}$ & 18 & 0 & - \\
\hline 4 & $\mathrm{CoBr}_{2}$ (pyimine) & THF & 0.1 & 60 & 1 & 0 & - \\
\hline 5 & $\mathrm{CoBr}_{2}$ (dppe) & $\mathrm{CH}_{2} \mathrm{Cl}_{2}$ & 0.1 & 0 to $\mathrm{rt}$ & 18 & 0 & - \\
\hline 6 & $\mathrm{CoBr}_{2}$ (dppe) & THF & 1.0 & 60 & 18 & $>99$ & $>99: 1$ \\
\hline 7 & $\mathrm{CoBr}_{2}$ (pyimine) & $\mathrm{CH}_{2} \mathrm{Cl}_{2}$ & 1.0 & 40 & 18 & 0 & - \\
\hline 8 & $\mathrm{CoBr}_{2}\left(\mathrm{Cy}_{2}\right.$ diimine $)$ & $\mathrm{MeCN}$ & 1.0 & 60 & 18 & 5 & - \\
\hline 9 & $\mathrm{CoBr}_{2}$ (dppe) & $\mathrm{MeCN}$ & 1.0 & 60 & 1 & 10 & - \\
\hline 10 & $\mathrm{CoBr}_{2}$ (dppe) & THF & 0.1 & 0 & 1 & 0 & - \\
\hline 11 & $\mathrm{CoBr}_{2}$ (dppe) & THF & 0.5 & 40 & 2 & 34 & $>99: 1$ \\
\hline 12 & $\mathrm{CoBr}_{2}$ (dppe) & THF & 0.5 & 60 & 1 & 98 & $>99: 1$ \\
\hline 13 & $\mathrm{CoBr}_{2}$ (dppe) & THF & 0.1 & 40 & 2 & 75 & $>99: 1$ \\
\hline 14 & $\mathrm{CoBr}_{2}$ (dppe) & THF & 0.5 & $\mathrm{rt}$ & 1 & 47 & $93: 7$ \\
\hline 15 & $\mathrm{CoBr}_{2}$ (dppe) & THF & 1.0 & 40 & 2 & 53 & $97: 3$ \\
\hline
\end{tabular}

a) Reaction conditions: Cobalt catalyst (5 mol \%), Zn, Znl 2 (10 mol \% each), 1,3-butadiyne 13 (0.30 mmol, 1.0 equiv.), solvent $(0.3 \mathrm{~mL})$. Conversion and ratio were determined by ${ }^{19} \mathrm{~F}$ NMR spectroscopy with hexafluorobenzene as internal standard. $\mathrm{Cy}_{2}$ (diimine): dicyclohexylethane-1,2-diimine; pyimine: $N$-mesityl-1-(pyridin-2-yl)methanimine; dppe: 1,2-bis(diphenylphosphino)ethane. 


\section{Synthesis of Unsymmetrical substituted 1,3-Butadiynes}

\section{General Procedure 2: Synthesis of unsymmetrical substituted 1,3-butadyines}

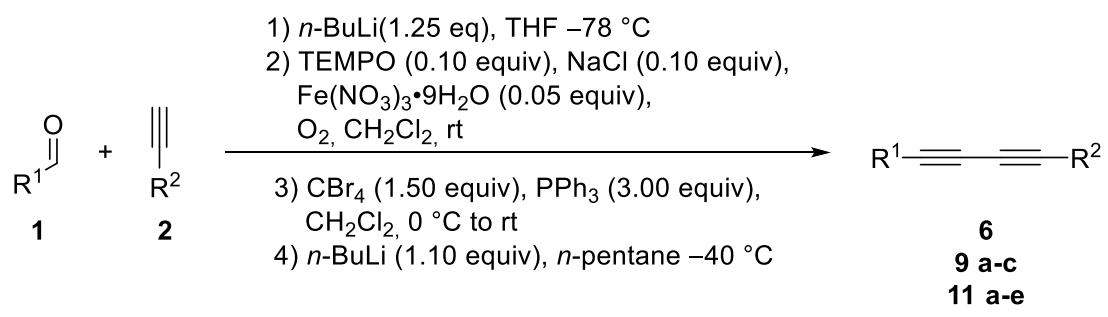

All unsymmetrical substituted diynes of type 6, 9 and 11 were synthesized following a slightly modified procedure of our group, which was reported earlier. ${ }^{2}$ In the oxidation reaction (step 2) the solvent was changed from 1,2-dichloroethane to dichloromethane. In the rearrangement reaction (step 4) the solvent was changed from $n$-hexane to $n$-pentane.

\section{((4-Fluorophenyl)buta-1,3-diyn-1-yl)trimethylsilane (6)}

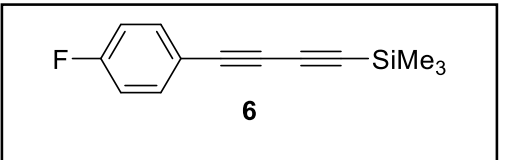

According to General Procedure 2 the title compound was prepared using 4-fluorobenzaldehyde $(2.48 \mathrm{~g}, 20.0 \mathrm{mmol}, 1.00$ equiv.), trimethylsilyl acetylene ( $3.40 \mathrm{~mL}, 25.0 \mathrm{mmol}, 1.25$ equiv.) and $n$-BuLi (2.5M in hexane, $10.0 \mathrm{~mL}, 1.25$ equiv.) in $40.0 \mathrm{~mL}$ THF. For the oxidation, TEMPO ( $0.32 \mathrm{~g}, 2.00 \mathrm{mmol}, 0.10$ equiv.), $\mathrm{NaCl}(0.16 \mathrm{~g}$, $2.00 \mathrm{mmol}, 0.10$ equiv.) and $\mathrm{Fe}\left(\mathrm{NO}_{3}\right)_{3} .9 \mathrm{H}_{2} \mathrm{O}\left(0.40 \mathrm{~g}, 1.00 \mathrm{mmol}, 0.05\right.$ equiv.) in $20.0 \mathrm{~mL} \mathrm{CH} 2 \mathrm{Cl}_{2}$ was used. For the dibromo-olefination tetrabromomethane $\left(9.95 \mathrm{~g}, 30.0 \mathrm{mmol}, 1.50\right.$ equiv.) and $\mathrm{PPh}_{3}(15.7 \mathrm{~g}, 60.0 \mathrm{mmol}, 3.00$ equiv.) in $80 \mathrm{~mL} \mathrm{CH}{ }_{2} \mathrm{Cl}_{2}$ was used. For the rearrangement $n$-BuLi (2.5M in hexane, $10.0 \mathrm{~mL}, 1.25$ equiv.) in $100 \mathrm{~mL}$ $n$-pentane was used. The product was obtained after column chromatography (eluent: $n$-pentane) as a colorless solid (4.00 g, $18.5 \mathrm{mmol}, 93 \%)$.

${ }^{1} \mathrm{H}$ NMR $\left(300 \mathrm{MHz}, \mathrm{CDCl}_{3}\right) \delta$ 7.51-7.43 (m, 2H), 7.06-6.96 (m, 2H), $0.23(\mathrm{~s}, 9 \mathrm{H}) \mathrm{ppm} .{ }^{13} \mathrm{C} \mathrm{NMR}\left(75 \mathrm{MHz}, \mathrm{CDCl}_{3}\right) \delta$ $163.2(\mathrm{~d}, J=251.8 \mathrm{~Hz}$ ), 134.9 (d, $J=8.6 \mathrm{~Hz}), 117.7$ (d, $J=3.6 \mathrm{~Hz}$ ), 116.0 (d, $J=22.2 \mathrm{~Hz}$ ), 90.9, 87.8, 75.8, 74.1, $-0.3 \mathrm{ppm} .{ }^{19} \mathrm{~F}$ NMR $\left(470 \mathrm{MHz}, \mathrm{CDCl}_{3}\right) \delta-110.8 \mathrm{ppm}$. IR (ATR) $\tilde{v}=2962,2207,2102,1893,1597,1504,1409,1289$, $1236,1155,1094,1019,834,760,670 \mathrm{~cm}^{-1}$. HRMS $\left(\mathrm{El}^{+}\right) \mathrm{m} / \mathrm{z}$ for $\mathrm{C}_{13} \mathrm{H}_{13} \mathrm{FSi}[\mathrm{M}]^{+}$: calc: 216.0765 , found: 216.0762. Melting point: $54-56^{\circ} \mathrm{C}$.

\section{1-Fluoro-4-(hexa-1,3-diyn-1-yl)benzene (9a)}

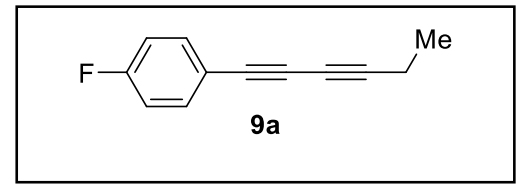

According to General Procedure 2 the title compound was prepared using propionaldehyde $(1.80 \mathrm{~g}, 20.0 \mathrm{mmol}, 1.00$ equiv.), 1-ethynyl-4fluorobenzene $(2.48 \mathrm{~g}, 25.00 \mathrm{mmol}, 1.25$ equiv.) and $n$-BuLi (2.5M in hexane, $10.0 \mathrm{~mL}, 1.25$ equiv.) in $40.0 \mathrm{~mL}$ THF. For the oxidation, TEMPO

( $0.32 \mathrm{~g}, 2.00 \mathrm{mmol}, 0.10$ equiv.), $\mathrm{NaCl}\left(0.16 \mathrm{~g}, 2.00 \mathrm{mmol}, 0.10\right.$ equiv.) and $\mathrm{Fe}\left(\mathrm{NO}_{3}\right)_{3} \cdot 9 \mathrm{H}_{2} \mathrm{O}(0.40 \mathrm{~g}, 1.00 \mathrm{mmol}$, 0.05 equiv.) in $20.0 \mathrm{~mL} \mathrm{CH}{ }_{2} \mathrm{Cl}_{2}$ was used. For the dibromo-olefination tetrabromomethane $(9.95 \mathrm{~g}, 30.0 \mathrm{mmol}$, 1.50 equiv.) and $\mathrm{PPh}_{3}\left(15.7 \mathrm{~g}, 60.0 \mathrm{mmol}, 3.00\right.$ equiv.) in $80 \mathrm{~mL} \mathrm{CH}{ }_{2} \mathrm{Cl}_{2}$ was used. For the rearrangement $n$-BuLi 
( $2.5 \mathrm{M}$ in hexane, $10.0 \mathrm{~mL}, 1.25$ equiv.) in $100 \mathrm{~mL} n$-pentane was used. The product was obtained after column chromatography (eluent: $n$-pentane) as a yellow oil (1.56 g, $8.32 \mathrm{mmol}, 48 \%)$.

${ }^{1} \mathrm{H}$ NMR $\left(300 \mathrm{MHz}, \mathrm{CDCl}_{3}\right) \delta 7.45(\mathrm{dd}, J=8.7,5.5 \mathrm{~Hz}, 1 \mathrm{H}), 7.00(\mathrm{t}, J=8.7 \mathrm{~Hz}, 1 \mathrm{H}), 2.37(\mathrm{q}, J=7.5, \mathrm{~Hz}, 2 \mathrm{H}), 1.21(\mathrm{t}$, $J=7.5 \mathrm{~Hz}, 3 \mathrm{H}) \mathrm{ppm} .{ }^{13} \mathrm{C} \mathrm{NMR}\left(75 \mathrm{MHz}, \mathrm{CDCl}_{3}\right) \delta 162.9(\mathrm{~d}, J=250.8 \mathrm{~Hz}), 134.6(\mathrm{~d}, J=8.5 \mathrm{~Hz}), 118.3(\mathrm{~d}, J=3.6 \mathrm{~Hz})$, $115.9(\mathrm{~d}, J=22.2 \mathrm{~Hz}), 86.0,74.2,73.9,64.5,13.4 \mathrm{ppm} .{ }^{19} \mathrm{~F} \mathrm{NMR}\left(470 \mathrm{MHz}, \mathrm{CDCl}_{3}\right) \delta-112.6 \mathrm{ppm}$.

IR (ATR) $\tilde{v}=2982,2941,2245,1722,1601,1506,1462,1431,1410,1379,1332,1296,1271,1231,1157,1094$, 1060, 1016, 834, 781, 763, 734, 645, $583 \mathrm{~cm}^{-1}$. HRMS $\left(\mathrm{EI}^{+}\right) \mathrm{m} / z$ for $\mathrm{C}_{12} \mathrm{H}_{9} \mathrm{~F}[\mathrm{M}]^{+}$: calc: 172.0683, found: 172.0677.

\section{1-Fluoro-4-(5-methylhexa-1,3-diyn-1-yl)benzene (9b)}

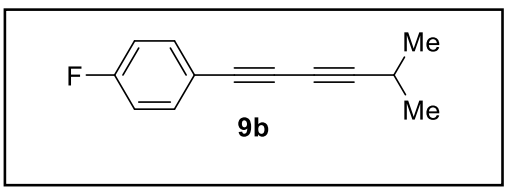

According to General Procedure 2 the title compound was prepared using 4-fluorobenzaldehyde ( $2.48 \mathrm{~g}, 20.0 \mathrm{mmol}, 1.00$ equiv.), 3-methyl-1-butyne (1.70 h, $25.00 \mathrm{mmol}, 1.25$ equiv.) and $n$-BuLi (2.5M in hexane, $10.0 \mathrm{~mL}, 1.25$ equiv.) in $40.0 \mathrm{~mL}$ THF. For the oxidation, TEMPO $(0.32 \mathrm{~g}, 2.00 \mathrm{mmol}, 0.10$ equiv.), $\mathrm{NaCl}\left(0.16 \mathrm{~g}, 2.00 \mathrm{mmol}, 0.10\right.$ equiv.) and $\mathrm{Fe}\left(\mathrm{NO}_{3}\right)_{3} \cdot 9 \mathrm{H}_{2} \mathrm{O}(0.40 \mathrm{~g}, 1.00 \mathrm{mmol}, 0.05$ equiv.) in $20.0 \mathrm{~mL}$ $\mathrm{CH}_{2} \mathrm{Cl}_{2}$ was used. For the dibromo-olefination tetrabromomethane (9.95 g, $30.0 \mathrm{mmol}, 1.50$ equiv.) and $\mathrm{PPh}_{3}$ (15.7 g, $60.0 \mathrm{mmol}, 3.00$ equiv.) in $80 \mathrm{~mL} \mathrm{CH}{ }_{2} \mathrm{Cl}_{2}$ was used. For the rearrangement $n$-BuLi (2.5M in hexane, 10.0 $\mathrm{mL}, 1.25$ equiv.) in $100 \mathrm{~mL} n$-pentane was used. The product was obtained after column chromatography (eluent: $n$-pentane) as a colorless solid (1.67 g, $8.99 \mathrm{mmol}, 45 \%)$.

${ }^{1} \mathrm{H}$ NMR $\left(300 \mathrm{MHz}, \mathrm{CDCl}_{3}\right) \delta 7.45(\mathrm{dd}, J=8.6,5.5 \mathrm{~Hz}, 2 \mathrm{H}), 7.00(\mathrm{t}, J=8.7 \mathrm{~Hz}, 2 \mathrm{H}), 2.71$ (hept, $\left.J=6.9 \mathrm{~Hz}, 1 \mathrm{H}\right), 1.25$ (s, 3H), 1.22 (s, 3H) ppm. $\left.{ }^{13} \mathrm{C} \mathrm{NMR} \mathrm{(75} \mathrm{MHz,} \mathrm{CDCl} 3\right) \delta 162.9(\mathrm{~d}, J=250.7 \mathrm{~Hz}), 134.6(\mathrm{~d}, J=8.5 \mathrm{~Hz}), 118.3$ (d, J = 3.5 $\mathrm{Hz}), 115.9$ (d, $J=22.2 \mathrm{~Hz}), 89.9,74.5,74.1,64.3,22.6,21.5$ ppm. ${ }^{19} \mathrm{~F}$ NMR $\left(470 \mathrm{MHz}, \mathrm{CDCl}_{3}\right) \delta-112.6$ ppm. IR (ATR) $\tilde{v}=3049,2975,2935,2874,2555,2239,2149,1894,1649,1594,1504,1468,1405,1384,1365,1329$, $1217,1170,1156,1094,1045,1011,953,944,888,832,734,648,586,557 \mathrm{~cm}^{-1}$. HRMS $\left(E I^{+}\right) \mathrm{m} / z$ for $\mathrm{C}_{13} \mathrm{H}_{11} \mathrm{~F}[\mathrm{M}]^{+}$: calc: 186.0839 , found: 186.0833 . Melting point: $47-49^{\circ} \mathrm{C}$.

\section{1-(5,5-Dimethylhexa-1,3-diyn-1-yl)-4-fluorobenzene (9c)}

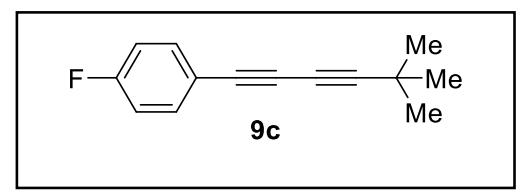

According to General Procedure 2 the title compound was prepared using 4-fluorobenzaldehyde ( $2.48 \mathrm{~g}, 20.0 \mathrm{mmol}, 1.00$ equiv.), 3,3-dimethyl-1butyne (1.97 g, $25.00 \mathrm{mmol}, 1.25$ equiv.) and $n$-BuLi (2.5M in hexane, 10.0 $\mathrm{mL}, 1.25$ equiv.) in $40.0 \mathrm{~mL}$ THF. For the oxidation, TEMPO (0.32 g, 2.00 mmol, 0.10 equiv.), $\mathrm{NaCl}\left(0.16 \mathrm{~g}, 2.00 \mathrm{mmol}, 0.10\right.$ equiv.) and $\mathrm{Fe}\left(\mathrm{NO}_{3}\right)_{3} \cdot 9 \mathrm{H}_{2} \mathrm{O}(0.40 \mathrm{~g}, 1.00 \mathrm{mmol}, 0.05$ equiv.) in $20.0 \mathrm{~mL} \mathrm{CH} \mathrm{Cl}_{2}$ was used. For the dibromo-olefination tetrabromomethane (9.95 g, $30.0 \mathrm{mmol}, 1.50$ equiv.) and $\mathrm{PPh}_{3}$ (15.7 g, $60.0 \mathrm{mmol}, 3.00$ equiv.) in $80 \mathrm{~mL} \mathrm{CH} \mathrm{Cl}_{2}$ was used. For the rearrangement $n$-BuLi (2.5M in hexane, $10.0 \mathrm{~mL}, 1.25$ equiv.) in $100 \mathrm{~mL} n$-pentane was used. The product was obtained after column chromatography (eluent: $n$-pentane) as a colorless solid (3.84 g, $19.2 \mathrm{mmol}, 96 \%)$.

${ }^{1} \mathbf{H}$ NMR $\left(300 \mathrm{MHz}, \mathrm{CDCl}_{3}\right) \delta 7.44(\mathrm{ddt}, J=8.1,5.0,2.4 \mathrm{~Hz}, 2 \mathrm{H}), 7.04-6.94(\mathrm{~m}, 2 \mathrm{H}), 1.29(\mathrm{~s}, 9 \mathrm{H})$ ppm. ${ }^{13} \mathrm{C}$ NMR $\left(75 \mathrm{MHz}, \mathrm{CDCl}_{3}\right) \delta 162.9(\mathrm{~d}, J=250.8 \mathrm{~Hz}), 134.5(\mathrm{~d}, J=8.5 \mathrm{~Hz}), 118.4(\mathrm{~d}, J=3.6 \mathrm{~Hz}), 115.9(\mathrm{~d}, J=22.2 \mathrm{~Hz}), 92.4$, 75.0, 74.0, 63.7, 30.6, 28.4 ppm. ${ }^{19} \mathrm{~F}$ NMR $\left(470 \mathrm{MHz}, \mathrm{CDCl}_{3}\right) \delta-122.6 \mathrm{ppm}$. IR (ATR) $\tilde{v}=2933,2868,2239,2147$, 
$1890,1598,1506,1480,1458,1406,1323,1296,1226,1203,1157,1096,1053,1014,933,879,853,832,698$, $568 \mathrm{~cm}^{-1}$. HRMS $\left(\mathrm{El}^{+}\right) \mathrm{m} / \mathrm{z}$ for $\mathrm{C}_{14} \mathrm{H}_{13} \mathrm{~F}[\mathrm{M}]^{+}$: calc: 200.0996 , found: 200.0990 . Melting point: $80-83^{\circ} \mathrm{C}$.

\section{((4-Methoxyphenyl)buta-1,3-diyn-1-yl)trimethylsilane (11a)}

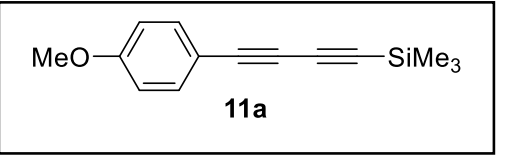

According to General Procedure 2 the title compound was prepared using 4-methoxybenzaldehyde (2.72 g, $20.0 \mathrm{mmol}, 1.00$ equiv.), trimethylsilyl acetylene ( $3.40 \mathrm{~mL}, 25.00 \mathrm{mmol}, 1.25$ equiv.) and $n$-BuLi (2.5M in hexane,

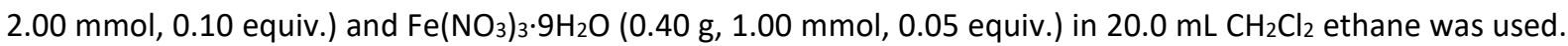
For the dibromo-olefination tetrabromomethane $\left(9.95 \mathrm{~g}, 30.0 \mathrm{mmol}, 1.50\right.$ equiv.) and $\mathrm{PPh}_{3}(15.7 \mathrm{~g}, 60.0 \mathrm{mmol}$, 3.00 equiv.) in $80 \mathrm{~mL} \mathrm{CH}{ }_{2} \mathrm{Cl}_{2}$ was used. For the rearrangement $n$-BuLi (2.5M in hexane, $10.0 \mathrm{~mL}, 1.25$ equiv.) in $100 \mathrm{~mL} n$-pentane was used. The product was obtained after column chromatography (eluent: $n$-pentane: $\mathrm{CH}_{2} \mathrm{Cl}_{2}$ $=10: 1)$ as a light-yellow oil (4.09 g, $17.9 \mathrm{mmol}, 89 \%)$.

${ }^{1} \mathrm{H}$ NMR $\left(300 \mathrm{MHz}, \mathrm{CDCl}_{3}\right) \delta 7.43(\mathrm{~d}, J=8.9 \mathrm{~Hz}, 2 \mathrm{H}), 6.83(\mathrm{~d}, J=8.9 \mathrm{~Hz}, 2 \mathrm{H}), 3.81(\mathrm{~s}, 3 \mathrm{H}), 0.23(\mathrm{~s}, 9 \mathrm{H}) \mathrm{ppm}$.

${ }^{13} \mathrm{C} \mathrm{NMR}\left(75 \mathrm{MHz}, \mathrm{CDCl}_{3}\right) \delta 160.6,134.5,114.3,113.4,90.0,88.3,73.2,55.5,-0.2 \mathrm{ppm}$. The analytical data are in accordance with the literature. ${ }^{2}$

\section{((3-Methoxyphenyl)buta-1,3-diyn-1-yl)trimethylsilane (11b)}

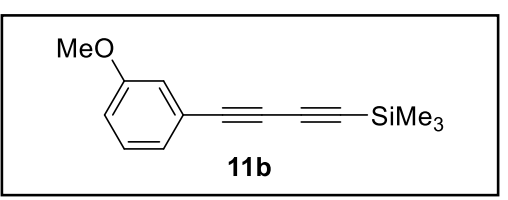

According to General Procedure 2 the title compound was prepared using 3-methoxybenzaldehyde (2.72 g, $20.0 \mathrm{mmol}, 1.00$ equiv.), trimethylsilyl acetylene ( $3.40 \mathrm{~mL}, 25.00 \mathrm{mmol}, 1.25$ equiv.) and $n$-BuLi (2.5M in hexane, $10.0 \mathrm{~mL}, 1.25$ equiv.) in $40.0 \mathrm{~mL}$ THF. For the oxidation, TEMPO (0.32 g, 2.0 mmol, 0.10 equiv.), $\mathrm{NaCl}\left(0.16 \mathrm{~g}, 2.00 \mathrm{mmol}, 0.10\right.$ equiv.) and $\mathrm{Fe}\left(\mathrm{NO}_{3}\right)_{3} \cdot 9 \mathrm{H}_{2} \mathrm{O}(0.40 \mathrm{~g}, 1.00 \mathrm{mmol}, 0.05$ equiv.) in $20.0 \mathrm{~mL} \mathrm{CH} \mathrm{Cl}_{2}$ was used. For the dibromo-olefination tetrabromomethane (9.95 g, $30.0 \mathrm{mmol}, 1.50$ equiv.) and $\mathrm{PPh}_{3}\left(15.7 \mathrm{~g}, 60.0 \mathrm{mmol}, 3.00\right.$ equiv.) in $80 \mathrm{~mL} \mathrm{CH} \mathrm{Cl}_{2}$ was used. For the rearrangement $n$-BuLi (2.5M in hexane, $10.0 \mathrm{~mL}, 1.25$ equiv.) in $100 \mathrm{~mL} n$-pentane was used. The product was obtained after column chromatography (eluent: $n$-pentane: $\mathrm{CH}_{2} \mathrm{Cl}_{2}=10: 1$ ) as a yellow oil $(3.85 \mathrm{~g}, 18.5 \mathrm{mmol}, 85 \%)$.

${ }^{1}{ }^{H}$ NMR $\left(300 \mathrm{MHz} \mathrm{CDCl}_{3}\right) \delta$ 7.29-7.21 (m, 1H), $7.11(\mathrm{dt}, J=7.6,1.2 \mathrm{~Hz}, 1 \mathrm{H}), 7.03(\mathrm{dd}, J=2.6,1.4 \mathrm{~Hz}, 1 \mathrm{H}), 6.94$ (ddd, $J=8.3,2.7,1.1 \mathrm{~Hz}, 1 \mathrm{H}), 3.81$ (s, 3H), 0.26 (s, 9H) ppm. ${ }^{13} \mathrm{C} \mathrm{NMR}\left(75 \mathrm{MHz}, \mathrm{CDCl}_{3}\right) \delta$ 159.5, 129.7, 125.4, 122.5, $117.4,116.3,90.9,88.0,76.8,74.1,55.4,-0.2 \mathrm{ppm}$. The analytical data are in accordance with the literature. ${ }^{2}$

\section{((3-Methoxyphenyl)buta-1,3-diyn-1-yl)trimethylsilane (11c)}

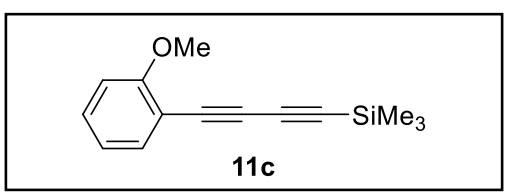

According to General Procedure 2 the title compound was prepared using 2-methoxybenzaldehyde ( $2.72 \mathrm{~g}, 20.0 \mathrm{mmol}, 1.00$ equiv.), trimethylsilyl acetylene ( $3.40 \mathrm{~mL}, 25.00 \mathrm{mmol}, 1.25$ equiv.) and $n$-BuLi (2.5M in hexane, $10.0 \mathrm{~mL}, 1.25$ equiv.) in $40.0 \mathrm{~mL}$ THF. For the oxidation, TEMPO (0.32 g, $2.00 \mathrm{mmol}, 0.10$ equiv.), $\mathrm{NaCl}\left(0.16 \mathrm{~g}, 2.00 \mathrm{mmol}, 0.10\right.$ equiv.) and $\mathrm{Fe}\left(\mathrm{NO}_{3}\right)_{3} \cdot 9 \mathrm{H}_{2} \mathrm{O}(0.40 \mathrm{~g}, 1.00 \mathrm{mmol}, 0.05$ equiv.) in $20.0 \mathrm{~mL} \mathrm{CH}{ }_{2} \mathrm{Cl}_{2}$ was used. For the dibromo-olefination tetrabromomethane (9.95 g, $30.0 \mathrm{mmol}, 1.50$ equiv.) and $\mathrm{PPh}_{3}$ (15.7 g, 60.0 mmol, 3.00 equiv.) in $80 \mathrm{~mL} \mathrm{CH} \mathrm{Cl}_{2}$ was used. For the rearrangement $n$-BuLi (2.5M in 
hexane, $10.0 \mathrm{~mL}, 1.25$ equiv.) in $200 \mathrm{~mL} n$-pentane was used. The product was obtained after column chromatography (eluent: $n$-pentane: $\mathrm{CH}_{2} \mathrm{Cl}_{2}=10: 1$ ) as a yellow oil (2.7 $\left.7 \mathrm{~g}, 12.2 \mathrm{mmol}, 61 \%\right)$.

${ }^{1} \mathrm{H}$ NMR $\left(500 \mathrm{MHz}, \mathrm{CDCl}_{2}\right) \delta 7.44(\mathrm{dd}, J=7.6,1.8 \mathrm{~Hz}, 1 \mathrm{H}), 7.32$ (ddd, $\left.J=8.4,7.5,1.7 \mathrm{~Hz}, 1 \mathrm{H}\right), 6.91-6.84(\mathrm{~m}, 2 \mathrm{H})$, 3.88 (s, 3H), 0.22 (s, 9H) ppm. ${ }^{13} \mathrm{C}$ NMR $\left(126 \mathrm{MHz} \mathrm{CDCl}_{3}\right) \delta 161.8,134.8,130.9,120.6,110.8,91.2,88.2,78.0$, $73.5,55.9,-0.2 \mathrm{ppm}$. The analytical data are in accordance with the literature. ${ }^{2}$

\section{((3-Methoxyphenyl)buta-1,3-diyn-1-yl)triisopropylsilane}

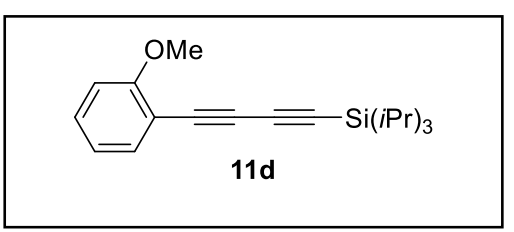

According to General Procedure 2 the title compound was prepared using 2-methoxybenzaldehyde (1.36 g, $10.0 \mathrm{mmol}, 1.00$ equiv.), triisopropylsilylacetylene $(2.28 \mathrm{~g}, 12.5 \mathrm{mmol}, 1.25$ equiv.) and $n$-BuLi (1.6M in hexane, $7.8 \mathrm{~mL}, 1.25$ equiv.) in $20.0 \mathrm{~mL}$ THF. For the oxidation, TEMPO

( $0.16 \mathrm{~g}, 1.00 \mathrm{mmol}, 0.10$ equiv.), $\mathrm{NaCl}\left(0.08 \mathrm{~g}, 1.00 \mathrm{mmol}, 0.10\right.$ equiv.) and $\mathrm{Fe}\left(\mathrm{NO}_{3}\right)_{3} \cdot 9 \mathrm{H}_{2} \mathrm{O}(0.20 \mathrm{~g}, 0.50 \mathrm{mmol}$, 0.05 equiv.) in $10.0 \mathrm{~mL} \mathrm{CH} \mathrm{Cl}_{2}$ was used. For the dibromo-olefination tetrabromomethane $(4.97 \mathrm{~g}, 15.0 \mathrm{mmol}$, 1.50 equiv.) and $\mathrm{PPh}_{3}$ (7.87 g, $30.0 \mathrm{mmol}, 3.00$ equiv.) in $40 \mathrm{~mL} \mathrm{CH} \mathrm{Cl}_{2}$ was used. For the rearrangement $n$-BuLi (1.6M in hexane, $6.9 \mathrm{~mL}, 1.10$ equiv.) in $100 \mathrm{~mL} n$-pentane was used. The product was obtained after column chromatography (eluent: $n$-pentane: $\mathrm{CH}_{2} \mathrm{Cl}_{2}=10: 1$ ) as a yellow solid (2.59 $\left.\mathrm{g}, 8.3 \mathrm{mmol}, 83 \%\right)$.

${ }^{1} \mathrm{H}$ NMR $\left(300 \mathrm{MHz}, \mathrm{CDCl}_{3}\right) \delta 7.48(\mathrm{dd}, J=7.6,1.7 \mathrm{~Hz}, 1 \mathrm{H}), 7.32$ (ddd, J= 8.4, 7.5, $\left.1.7 \mathrm{~Hz}, 1 \mathrm{H}\right), 6.94-6.84(\mathrm{~m}, 2 \mathrm{H})$, $3.89(\mathrm{~s}, 3 \mathrm{H}), 1.11(\mathrm{~s}, 21 \mathrm{H}) \mathrm{ppm} .{ }^{13} \mathrm{C}$ NMR $\left(75 \mathrm{MHz}, \mathrm{CDCl}_{3}\right) \delta 161.7,134.9,130.8,120.7,110.9,110.7,89.8,88.4$, 78.6, 72.1, 56.0, 18.7, 11.5 ppm. IR (ATR) $\tilde{v}=2943,2865,2201,2139,2099,1594,1573,1491,1461,1433,1385$,

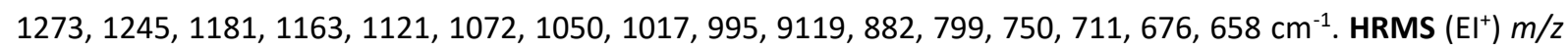
for $\mathrm{C}_{20} \mathrm{H}_{28} \mathrm{OSi}[\mathrm{M}]^{+}$: calc: 312.1904 , found: 312.1894 . Melting point: $34-37^{\circ} \mathrm{C}$.

\section{Trimethyl(nona-1,3-diyn-1-yl)silane (11e)}

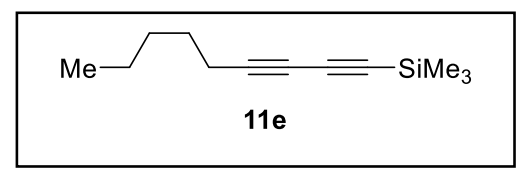

According to General Procedure 2 the title compound was prepared using hexanal ( $2.00 \mathrm{~g}, 20.0 \mathrm{mmol}, 1.00$ equiv.), trimethylsilyl acetylene $(3.40 \mathrm{~mL}$, $25.00 \mathrm{mmol}, 1.25$ equiv.) and $n$-BuLi (2.5M in hexane, $10.0 \mathrm{~mL}, 1.25$ equiv.)

in $40.0 \mathrm{~mL}$ THF. For the oxidation, TEMPO (0.32 g, $2.0 \mathrm{mmol}, 0.10$ equiv.), $\mathrm{NaCl}(0.16 \mathrm{~g}, 2.00 \mathrm{mmol}, 0.10$ equiv.) and $\mathrm{Fe}\left(\mathrm{NO}_{3}\right)_{3} \cdot 9 \mathrm{H}_{2} \mathrm{O}\left(0.40 \mathrm{~g}, 1.00 \mathrm{mmol}, 0.05\right.$ equiv.) in $20.0 \mathrm{~mL} \mathrm{CH} \mathrm{Cl}_{2}$ was used. For the dibromo-olefination tetrabromomethane (9.95 g, $30.0 \mathrm{mmol}, 1.50$ equiv.) and $\mathrm{PPh}_{3}\left(15.7 \mathrm{~g}, 60.0 \mathrm{mmol}, 3.00\right.$ equiv.) in $80 \mathrm{~mL} \mathrm{CH} \mathrm{Cl}_{2}$ was used. For the rearrangement $n$-BuLi (2.5M in hexane, $10.0 \mathrm{~mL}, 1.25$ equiv.) in $100 \mathrm{~mL} n$-pentane was used. The product was obtained after column chromatography (eluent: $n$-pentane) as a light-yellow oil (2.80 g, 14.5 $\mathrm{mmol}, 73 \%)$.

${ }^{1} \mathrm{H}$ NMR $\left(300 \mathrm{MHz}, \mathrm{CDCl}_{3}\right) \delta 2.27(\mathrm{t}, J=7.0 \mathrm{~Hz}, 2 \mathrm{H}), 1.52(\mathrm{td}, J=9.3,8.1,6.0 \mathrm{~Hz}, 2 \mathrm{H}), 1.43-1.22(\mathrm{~m}, 4 \mathrm{H}), 0.89(\mathrm{t}, J$ $=7.0 \mathrm{~Hz}, 3 \mathrm{H}), 0.18(\mathrm{~s}, 9 \mathrm{H}) \mathrm{ppm} .{ }^{13} \mathrm{C}$ NMR $\left(75 \mathrm{MHz}, \mathrm{CDCl}_{3}\right) \delta 88.6,83.1,80.4,65.6,31.1,28.0,22.3,19.4,14.0,-0.2$ ppm. The analytical data are in accordance with the literature. ${ }^{2}$ 


\section{Synthesis of Symmetrical 1,3-Butadiynes}

General Procedure 3: Synthesis of symmetrical substituted 1,3-butadyines via a Glaser-Hay coupling reaction

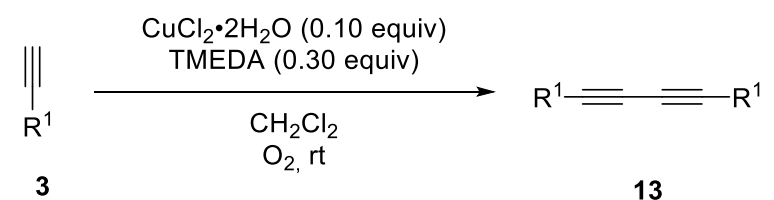

According to a literature known procedure $\mathrm{CuCl}_{2} \cdot 2 \mathrm{H}_{2} \mathrm{O}(10 \mathrm{~mol} \%)$ was suspended in $\mathrm{CH}_{2} \mathrm{Cl}_{2}(1 \mathrm{~mL} / \mathrm{mmol})$. To this suspension TMEDA ( 0.30 equiv.) was added. The suspension turned into a blue-green solution. Then the corresponding alkyne (1.00 equiv.) was added. The reaction mixture was stirred at ambient temperature under an atmosphere of oxygen until complete conversion was determined via GC/MS. The reaction mixture was diluted with $\mathrm{CH}_{2} \mathrm{Cl}_{2}$ and washed with $\mathrm{H}_{2} \mathrm{O}$ until the blue color of the aqueous phase disappeared. The aqueous phase was extracted with $\mathrm{CH}_{2} \mathrm{Cl}_{2}$ (three times). The combined organic layers were dried over $\mathrm{MgSO}_{4}$, filtered and the solvent was removed under reduced pressure. The obtained crude products were in most cases of high purity so that no further purification was needed.

\section{1,4-Bis(4-fluorophenyl)buta-1,3-diyne (13a)}

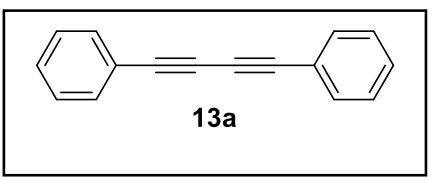

According to General Procedure 3 the title compound was prepared using phenylacetylene (9.3 g, $91.1 \mathrm{mmol}, 1.00$ equiv), $\mathrm{CuCl}_{2} \cdot 2 \mathrm{H}_{2} \mathrm{O}(0.17 \mathrm{~g}, 1.00 \mathrm{mmol}$, 0.01 equiv.), TMEDA ( $0.45 \mathrm{~mL}, 3.00 \mathrm{mmol}, 0.03$ equiv.) in $15.0 \mathrm{~mL} \mathrm{CH} \mathrm{Cl}_{2}$. The product was obtained as a colorless solid $(6.82 \mathrm{~g}, 41.5 \mathrm{mmol}, 91 \%)$.

${ }^{1} \mathbf{H}$ NMR $\left(300 \mathrm{MHz}_{\mathrm{CDCl}}\right) \delta 7.59-7.52\left(\mathrm{~m}, 4 \mathrm{H9}, 7,45-7.31(6 \mathrm{H}) \mathrm{ppm} .{ }^{13} \mathrm{C} \mathrm{NMR}\left(75 \mathrm{MHz}, \mathrm{CDCl}_{3}\right) \delta 132.7,129.3\right.$, 128.6, 122.0, 81.7, 74.1.ppm. The analytical data are in accordance with the literature. ${ }^{3}$

\section{1,4-Bis(4-fluorophenyl)buta-1,3-diyne (13b)}

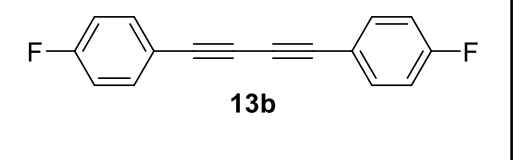

According to General Procedure 3 the title compound was prepared using 1-ethinyl-4-fluorobenzene ( $1.15 \mathrm{~g}, 9.57 \mathrm{mmol}, 1.00$ equiv), $\mathrm{CuCl}_{2} \cdot 2 \mathrm{H}_{2} \mathrm{O}$ (0.17 g, $1.00 \mathrm{mmol}, 0.10$ equiv.), TMEDA (0.45 mL, $3.00 \mathrm{mmol}, 0.31$ equiv.) in $10.0 \mathrm{~mL} \mathrm{CH} \mathrm{Cl}_{2}$. The product was obtained as a light brown solid (1.04 g, $4.35 \mathrm{mmol}, 91 \%$ ).

${ }^{1} \mathrm{H}$ NMR $\left(300 \mathrm{MHz}, \mathrm{CDCl}_{3}\right) \delta 7.51(\mathrm{dd}, J=8.7,5.5 \mathrm{~Hz}, 4 \mathrm{H}), 7.04(\mathrm{t}, J=8.7 \mathrm{~Hz}, 4 \mathrm{H}) \mathrm{ppm} .{ }^{13} \mathrm{C} \mathrm{NMR}\left(75 \mathrm{MHz}, \mathrm{CDCl}_{3}\right) \delta$ $163.2(\mathrm{~d}, J=251.6 \mathrm{~Hz}), 134.7(\mathrm{~d}, J=8.5 \mathrm{~Hz}), 117.9(\mathrm{~d}, J=3.7 \mathrm{~Hz}), 116.1(\mathrm{~d}, J=22.3 \mathrm{~Hz}), 80.6,73.7 \mathrm{ppm} .{ }^{19} \mathrm{~F} \mathrm{NMR}$ $\left(470 \mathrm{MHz}, \mathrm{CDCl}_{3}\right) \delta-111.7 \mathrm{ppm}$. The analytical data are in accordance with the literature. ${ }^{4}$

\section{1,4-Bis(4-chlorophenyl)buta-1,3-diyne (13c)}

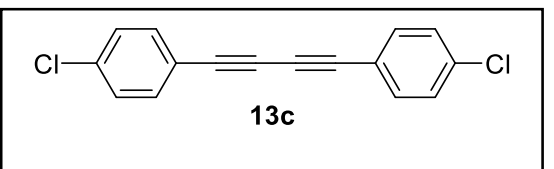

According to General Procedure 3 the title compound was prepared using 1-ethynyl-4-chlorobenzene (1.37 g, $10.0 \mathrm{mmol}, 1.00$ equiv.), $\mathrm{CuCl}_{2} \cdot 2 \mathrm{H}_{2} \mathrm{O}(0.17 \mathrm{~g}, 1.00 \mathrm{mmol}, 0.10$ equiv.), TMEDA $(0.45 \mathrm{~mL}, 3.00$ mmol, 0.30 equiv.) in $10.0 \mathrm{~mL} \mathrm{CH} \mathrm{Cl}_{2}$. The product was obtained as a colorless solid (1.35 g, $\left.4.99 \mathrm{mmol}, 99 \%\right)$. 
The product is very insoluble in common organic solvents.

${ }^{1} \mathrm{H}$ NMR (300 MHz, CDCl $) \delta 7.45(\mathrm{~d}, J=8.5 \mathrm{~Hz}, 4 \mathrm{H}), 7.32$ (d, $\left.J=8.5 \mathrm{~Hz}, 4 \mathrm{H}\right) \mathrm{ppm}$. IR (ATR) $\tilde{v}=1644,1582,1476$, 1392, 1264, 1087, 1005, 818, 784, 727, $629 \mathrm{~cm}^{-1}$. HRMS $\left(\mathrm{El}^{+}\right) \mathrm{m} / \mathrm{z}$ for $\mathrm{C}_{16} \mathrm{H}_{8} \mathrm{Cl}_{2}[\mathrm{M}]^{+}$: calc: 269.9998, found: 269.9996. Melting point: $276-279^{\circ} \mathrm{C}$. The analytical data are in accordance with the literature. ${ }^{5}$

\section{1,4-Bis(4-bromophenyl)buta-1,3-diyne (13d)}

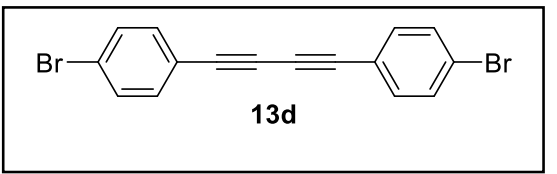

According to General Procedure 3 the title compound was prepared using 1-ethynyl-4-bromobenzene (1.81 g, $10.0 \mathrm{mmol}, 1.00$ equiv.), $\mathrm{CuCl}_{2} \cdot 2 \mathrm{H}_{2} \mathrm{O}$ (0.17 g, $1.00 \mathrm{mmol}, 0.10$ equiv.), TMEDA $(0.45 \mathrm{~mL}, 3.00$ mmol, 0.30 equiv.) in $10.0 \mathrm{~mL} \mathrm{CH} \mathrm{Cl}_{2}$. The product was obtained as a colorless solid $(1.80 \mathrm{~g}, 5.00 \mathrm{mmol},>99 \%)$. The product is very insoluble in common organic solvents.

${ }^{1} \mathbf{H}$ NMR $\left(300 \mathrm{MHz}, \mathrm{CDCl}_{3}\right) \delta 7.48(\mathrm{~d}, J=8.5 \mathrm{~Hz}, 4 \mathrm{H}), 7.38(\mathrm{~d}, J=8.5 \mathrm{~Hz}, 4 \mathrm{H}) \mathrm{ppm}$. IR (ATR) $\tilde{v}=1899,1642,1577$, 1478, 1389, 1266, 1226, 1095, 1066, 1006, 817, 776, 699, $640 \mathrm{~cm}^{-1}$. HRMS (EI+) m/z for $\mathrm{C}_{16} \mathrm{H}_{8} \mathrm{Br}_{2}\left[\mathrm{M}^{+}\right.$: calc: 357.8987, found: 357.8984 . Melting point: $285-289^{\circ} \mathrm{C}$. The analytical data are in accordance with the literature. ${ }^{5}$

\section{1,4-Bis(4-methoxyphenyl)buta-1,3-diyne (13e)}

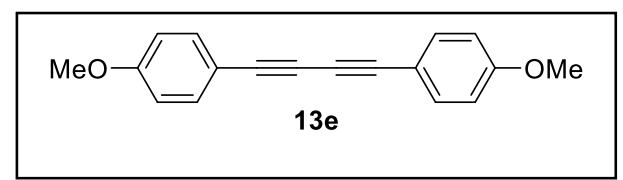

According to General Procedure 3 the title compound was prepared using 1-ethynyl-4-methoxybenzene $(1.32 \mathrm{~g}, 10.0 \mathrm{mmol}$, 1.00 equiv.), $\mathrm{CuCl}_{2} \cdot 2 \mathrm{H}_{2} \mathrm{O}$ ( $0.17 \mathrm{~g}, 1.00 \mathrm{mmol}, 0.10$ equiv.), TMEDA ( $0.45 \mathrm{~mL}, 3.00 \mathrm{mmol}, 0.30$ equiv.) in $10.0 \mathrm{~mL} \mathrm{CH}{ }_{2} \mathrm{Cl}_{2}$. The product was obtained as a light yellow solid (1.32 $\mathrm{g}, 5.00$ $\mathrm{mmol},>99 \%)$.

${ }^{1} \mathrm{H}$ NMR $\left(300 \mathrm{MHz}, \mathrm{CDCl}_{3}\right) \delta 7.46(\mathrm{~d}, J=8.7 \mathrm{~Hz}, 4 \mathrm{H}), 6.85(\mathrm{~d}, J=8.8 \mathrm{~Hz}, 4 \mathrm{H}), 3.82(\mathrm{~s}, 6 \mathrm{H}) \mathrm{ppm} .{ }^{13} \mathrm{C} \mathrm{NMR}(75 \mathrm{MHz}$, $\left.\mathrm{CDCl}_{3}\right) \delta 160.3,134.2,114.3,114.0,81.4,73.1 \mathrm{ppm}$. The analytical data are in accordance with the literature. ${ }^{6}$

\section{1,4-Di-p-tolylbuta-1,3-diyne (13f)}

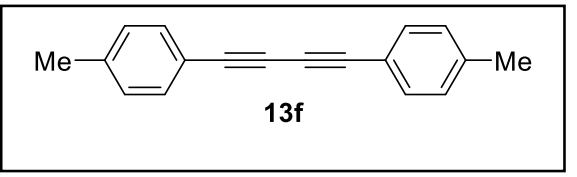

According to General Procedure 3 the title compound was prepared using 1-ethynyl-4-methylbenzene (1.16 g, $10.0 \mathrm{mmol}, 1.00$ equiv.), $\mathrm{CuCl}_{2} \cdot 2 \mathrm{H}_{2} \mathrm{O}$ (0.17 g, $1.00 \mathrm{mmol}, 0.10$ equiv.), TMEDA (0.45 mL, 3.00

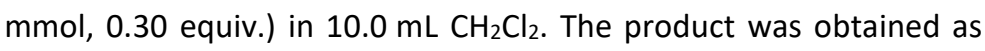
yellow solid (0.92 g, $3.98 \mathrm{mmol}, 80 \%)$.

${ }^{1} \mathbf{H}$ NMR $\left(300 \mathrm{MHz}, \mathrm{CDCl}_{3}\right) \delta 7.43(\mathrm{~d}, J=7.7 \mathrm{~Hz}, 2 \mathrm{H}), 7.15(\mathrm{~d}, J=7.7 \mathrm{~Hz}, 2 \mathrm{H}), 2.37(\mathrm{~s}, 3 \mathrm{H}) \mathrm{ppm} .{ }^{13} \mathrm{C} \mathrm{NMR}(75 \mathrm{MHz}$, $\left.\mathrm{CDCl}_{3}\right) \delta 139.6,132.5,129.3,118.9,81.7,73.6,21.8 \mathrm{ppm}$. The analytical data are in accordance with the literature. $^{7}$

\section{1,4-Bis(4-nitrophenyl)buta-1,3-diyne (13g)}

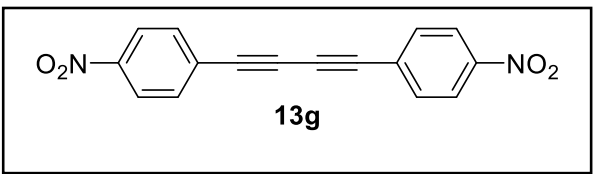

According to General Procedure 3 the title compound was prepared using 1-ethynyl-4-nitrobenzene (1.47 g, $10.0 \mathrm{mmol}, 1.00$ equiv.), $\mathrm{CuCl}_{2} \cdot 2 \mathrm{H}_{2} \mathrm{O}$ (0.17 g, $1.00 \mathrm{mmol}, 0.10$ equiv.), TMEDA (0.45 mL, 3.00 mmol, 0.30 equiv.) in $10.0 \mathrm{~mL} \mathrm{CH} \mathrm{Cl}_{2}$. The product was obtained as a yellow solid $(1.80 \mathrm{~g}, 5.00 \mathrm{mmol},>99 \%)$. 
The product is very insoluble in common organic solvents.

${ }^{1} \mathrm{H}$ NMR $\left(300 \mathrm{MHz}, \mathrm{CDCl}_{3}\right) \delta 8.24(\mathrm{~d}, J=8.5 \mathrm{~Hz}, 4 \mathrm{H}), 7.70(\mathrm{~d}, J=8.5 \mathrm{~Hz}, 4 \mathrm{H}) \mathrm{ppm}$. IR (ATR) $\tilde{v}=1589,1503,1367$, 1334, 1312, 1286, 1103, 951, 844, 766, 741, 670, $619 \mathrm{~cm}^{-1}$. HRMS (El+) m/z for $\mathrm{C}_{16} \mathrm{H}_{8} \mathrm{~N}_{2} \mathrm{O}_{4}[\mathrm{M}]^{+}:$calc: 292.0479 , found: 292.0479 . The analytical data are in accordance with the literature. ${ }^{8}$

\section{1,4-Di-m-tolylbuta-1,3-diyne (13h)}

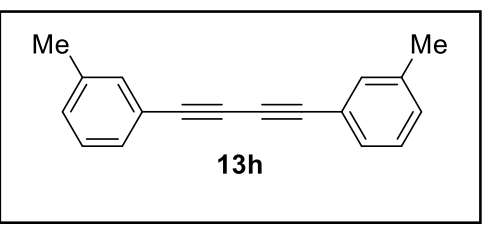

According to General Procedure 3 the title compound was prepared using 1-ethynyl-3-methylbenzene (1.16 g, $10.0 \mathrm{mmol}, 1.00$ equiv.), $\mathrm{CuCl}_{2} \cdot 2 \mathrm{H}_{2} \mathrm{O}$ ( $0.17 \mathrm{~g}, 1.00 \mathrm{mmol}, 0.10$ equiv.), TMEDA ( $0.45 \mathrm{~mL}, 3.00 \mathrm{mmol}, 0.30$ equiv.) in $10.0 \mathrm{~mL} \mathrm{CH} \mathrm{Cl}_{2}$. The product was obtained as a yellow solid (1.15 g, 4.99

mmol, 99\%).

${ }^{1} \mathrm{H}$ NMR $\left(300 \mathrm{MHz}, \mathrm{CDCl}_{3}\right) \delta$ 7.40-7.31 (m, 4H), 7.28-7.15 (m, 4H), $2.35(\mathrm{~s}, 6 \mathrm{H}) \mathrm{ppm} .{ }^{13} \mathrm{C} \mathrm{NMR}\left(75 \mathrm{MHz}, \mathrm{CDCl}_{3}\right) \delta$ $138.3,133.1,130.3,129.7,128.5,121.8,81.8,73.8,21.4$ ppm. The analytical data are in accordance with the literature. ${ }^{9}$

\section{1,4-Bis(3-methoxyphenyl)buta-1,3-diyne (13i)}

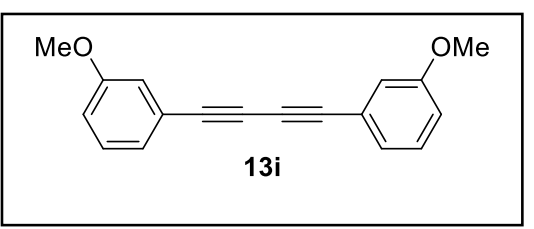

According to General Procedure 3 the title compound was prepared using 1-ethynyl-3-methoxybenzene (1.32 g, $10.0 \mathrm{mmol}, 1.00$ equiv.), $\mathrm{CuCl}_{2} \cdot 2 \mathrm{H}_{2} \mathrm{O}$ (0.17 g, $1.00 \mathrm{mmol}, 0.10$ equiv.), TMEDA $(0.45 \mathrm{~mL}, 3.00 \mathrm{mmol}$, 0.30 equiv.) in $10.0 \mathrm{~mL} \mathrm{CH} \mathrm{Cl}_{2}$. The product was obtained as yellow oil

(1.13 g, $4.31 \mathrm{mmol}, 86 \%)$.

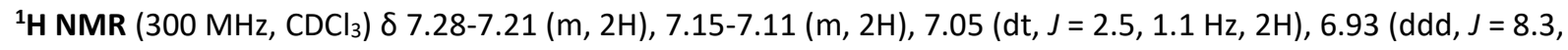
2.5, $1.2 \mathrm{~Hz}, 2 \mathrm{H}$ ), 3.81 (s, 6H) ppm. ${ }^{13} \mathrm{C}$ NMR $\left(75 \mathrm{MHz}, \mathrm{CDCl}_{3}\right) \delta 159.4,129.7,125.2,122.8,117.2,116.2,81.6,73.8$, $55.5 \mathrm{ppm}$. The analytical data are in accordance with the literature. ${ }^{4}$

\section{1,4-Bis(trimethylsilyl)buta-1,3-diyne (13j)}

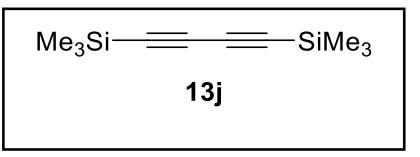

According to General Procedure 1 the title compound was prepared using trimethylsilyl acetylene (1.96 g, $20.0 \mathrm{mmol}, 1.00$ equiv.), $\mathrm{CuCl}_{2} \cdot 2 \mathrm{H}_{2} \mathrm{O}(0.34 \mathrm{~g}, 2.00$ mmol, 0.10 equiv.), TMEDA (0.90 mL, $6.00 \mathrm{mmol}, 0.60$ equiv.) in $20.0 \mathrm{~mL} \mathrm{CH} 2 \mathrm{Cl}_{2}$. The product was obtained as a dark-brown crystalline solid (1.62 g, $8.34 \mathrm{mmol}, 83 \%)$.

${ }^{1} \mathrm{H}$ NMR $\left(300 \mathrm{MHz}, \mathrm{CDCl}_{3}\right) \delta 0.19(\mathrm{~s}, 18 \mathrm{H}) \mathrm{ppm} .{ }^{13} \mathrm{C} \mathrm{NMR}\left(75 \mathrm{MHz}, \mathrm{CDCl}_{3}\right) \delta 88.1,86.1,-0.4 \mathrm{ppm}$. The analytical data are in accordance with the literature. ${ }^{10}$

\section{Deca-4,6-diyne (13k)}

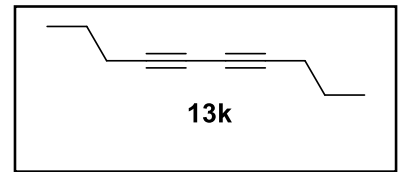

Following a modified procedure by Dickinson et al. ${ }^{11} \mathrm{PdCl}_{2}\left(\mathrm{PPh}_{3}\right)_{2}(0.42 \mathrm{~g}, 0.6 \mathrm{mmol}$, 0.03 equiv.), copper(I) iodide $(0.12 \mathrm{~g}, 0.12 \mathrm{mmol}, 0.06$ equiv.) and triphenylphosphine $(0.47 \mathrm{~g}, 1.8 \mathrm{mmol}, 0.09$ equiv.) were dissolved in acetonitrile $(20.0 \mathrm{~mL})$ and triethylamine $(8.00 \mathrm{~mL}, 60.0 \mathrm{mmol}, 3.00$ equiv.). After 15 minutes, 1-pentyne $(2.00 \mathrm{~mL}, 20.0 \mathrm{mmol}$, 1.00 equiv.) was added and the reaction mixture was stirred for $18 \mathrm{~h}$ at $60^{\circ} \mathrm{C}$. To the suspension was added brine. 
The aqueous layer was extracted three times with $\mathrm{Et}_{2} \mathrm{O}$. The combined organic layers were dried over $\mathrm{MgSO}_{4}$, filtered and the solvent was removed. The product was obtained after column chromatography (eluent: $n$-pentane) as a colorless liquid (470 $\mathrm{mg}, 3.50 \mathrm{mmol}, 35 \%)$.

${ }^{1} \mathrm{H}$ NMR $\left(300 \mathrm{MHz}, \mathrm{CDCl}_{3}\right) \delta 2.23(\mathrm{t}, J=7.1 \mathrm{~Hz}, 4 \mathrm{H}), 1.55(\mathrm{~h}, J=7.2 \mathrm{~Hz}, 4 \mathrm{H}), 0.98(\mathrm{t}, J=7.4 \mathrm{~Hz}, 6 \mathrm{H}) \mathrm{ppm} .{ }^{13} \mathrm{C} \mathrm{NMR}$ $\left(75 \mathrm{MHz}, \mathrm{CDCl}_{3}\right) \delta 77.5,65.522 .0,21.3,13.6 \mathrm{ppm}$. The analytical data are in accordance with the literature. ${ }^{11}$

\section{1,4-Di-o-tolylbuta-1,3-diyne (13I)}

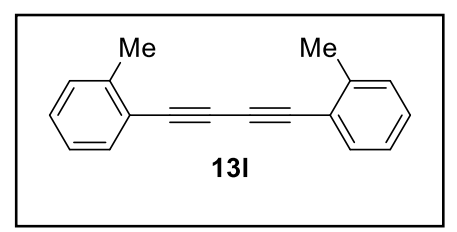

According to General Procedure 3 the title compound was prepared using 1-ethynyl-2-methylbenzene ( $1.16 \mathrm{~g}, 10.0 \mathrm{mmol}, 1.00$ equiv.), $\mathrm{CuCl}_{2} \cdot 2 \mathrm{H}_{2} \mathrm{O}(0.17$ g, $1.00 \mathrm{mmol}, 0.10$ equiv.), TMEDA ( $0.45 \mathrm{~mL}, 3.00 \mathrm{mmol}, 0.30$ equiv.) in $10.0 \mathrm{~mL}$ $\mathrm{CH}_{2} \mathrm{Cl}_{2}$. The product was obtained as a yellow solid (1.11 g, $\left.4.83 \mathrm{mmol}, 97 \%\right)$.

${ }^{1} \mathbf{H}$ NMR $\left(300 \mathrm{MHz}_{2} \mathrm{CDCl}_{3}\right) \delta 7.54(\mathrm{~d}, J=7.6 \mathrm{~Hz}, 1 \mathrm{H}), 7.36-7.20(\mathrm{~m}, 1 \mathrm{H}), 7.22-7.15(\mathrm{~m}, 1 \mathrm{H}), 2.53(\mathrm{~s}, 3 \mathrm{H}) \mathrm{ppm}$. ${ }^{13} \mathrm{C} \mathrm{NMR}\left(75 \mathrm{MHz}, \mathrm{CDCl}_{3}\right) \delta 141.8,133.1,129.7,129.2,125.8,121.9,81.3,77.7,20.9 \mathrm{ppm}$. The analytical data are in accordance with the literature. ${ }^{4}$

\section{1,4-Bis(2-methoxyphenyl)buta-1,3-diyne (13m)}

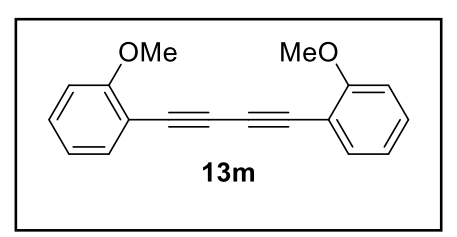

According to General Procedure 3 the title compound was prepared using 1-ethynyl-3-methoxybenzene ( $1.32 \mathrm{~g}, 10.0 \mathrm{mmol}, 1.00$ equiv.), $\mathrm{CuCl}_{2} \cdot 2 \mathrm{H}_{2} \mathrm{O}(0.17$ g, $1.00 \mathrm{mmol}, 0.10$ equiv.), TMEDA (0.45 mL, $3.00 \mathrm{mmol}, 0.30$ equiv.) in $10.0 \mathrm{~mL}$ $\mathrm{CH}_{2} \mathrm{Cl}_{2}$. The product was obtained as yellow solid (1.31 g, $\left.4.99 \mathrm{mmol}, 99 \%\right)$.

${ }^{1} \mathrm{H}$ NMR $\left(300 \mathrm{MHz}, \mathrm{CDCl}_{3}\right) \delta 7.48(\mathrm{dd}, J=7.5,1.7 \mathrm{~Hz}, 2 \mathrm{H}), 7.38-7.28(\mathrm{~m}, 2 \mathrm{H}), 6.97-6.84(\mathrm{~m}, 4 \mathrm{H}), 3.90(\mathrm{~s}, 6 \mathrm{H}) \mathrm{ppm}$. ${ }^{13} \mathrm{C}$ NMR $\left(75 \mathrm{MHz}, \mathrm{CDCl}_{3}\right) \delta 161.4,134.5,130.7,120.6,111.3,110.7,78.8,78.1,55.9 \mathrm{ppm}$. The analytical data are in accordance with the literature. ${ }^{4}$ 


\section{Cobalt-Catalyzed Cyclotrimerization of (Un)Symmetrical 1,3-}

\section{Butadiynes}

General Procedure 4: Cobalt-catalyzed [2+2+2] cyclotrimerization of 1,3-butadiynes to the 1,2,4-regioisomer

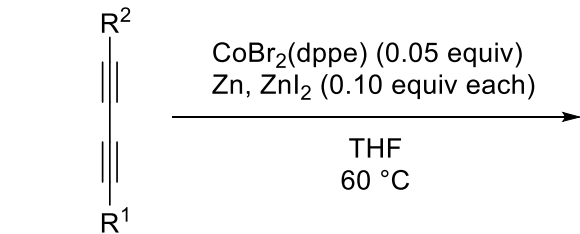

$R^{1} \neq R^{2}: 6,9,11$

$R^{1}=R^{2}: 13$

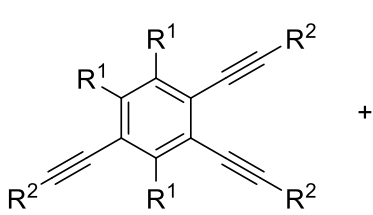

$7,10,12$

14<smiles>[R]C#Cc1c([R])c(C#C[R])c([R])c(C#C[R])c1[R]</smiles>

15

Under argon atmosphere $\mathrm{CoBr}_{2}$ (dppe) $(5 \mathrm{~mol} \%)$, zinc dust (10 mol \%) and zinc iodide (10 mol \%) were added to a pre-dried reaction vessel. The solids were dried for $15 \mathrm{~min}$ in vacuo. After that the catalyst system was suspended in dry THF $(1.0 \mathrm{~mL} / \mathrm{mmol})$ and the reaction mixture was stirred for $15 \mathrm{~min}$ at ambient temperature. Next the 1,3-butadiyne (1-10 mmol, 1.00 equiv.) was added and the reaction mixture was stirred at $60{ }^{\circ} \mathrm{C}$ until complete conversion was determined via GC/MS and TLC. The mixture was filtered over a short plug of silica gel (eluent: $n$-pentane: $\mathrm{CH}_{2} \mathrm{Cl}_{2}$ ) and the solvent was removed under reduced pressure. If needed, the crude products were purified via column chromatography (eluent: $n$-pentane: $\mathrm{CH}_{2} \mathrm{Cl}_{2}$ ).

((4,4"-Difluoro-5'-(4-fluorophenyl)-[1,1':2',1"'-terphenyl]-3',4',6'-triyl)tris(ethyne-2,1-diyl))tris(trimethylsilane) (7a)

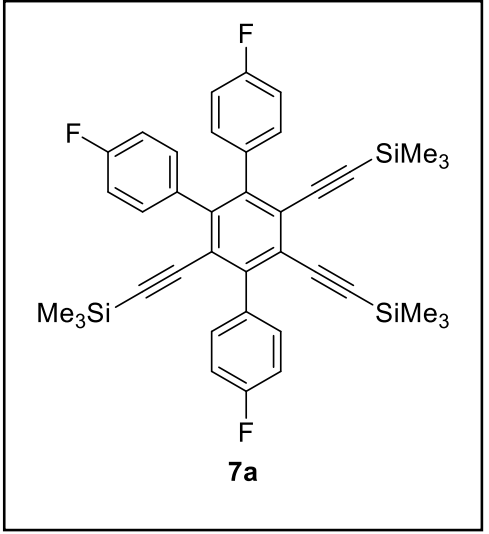

According to General Procedure 4 the title compound was prepared using ((4-fluorophenyl)buta-1,3-diyn-1-yl)trimethylsilane $(649 \mathrm{mg}, 3.00 \mathrm{mmol}$, 1.00 equiv.), $\mathrm{CoBr}_{2}$ (dppe) (92.6 mg, $0.15 \mathrm{mmol}, 0.05$ equiv.), zinc (19.6 mg, $0.30 \mathrm{mmol}, 0.10$ equiv.) and zinc(II)iodide ( $96.0 \mathrm{mg}, 0.30 \mathrm{mmol}, 0.10$ equiv.) in $3.00 \mathrm{~mL}$ THF. The product was obtained after filtration over a short plug of silica (eluent: $n$-pentane: $\mathrm{CH}_{2} \mathrm{Cl}_{2}=2: 1$ ) as a beige solid $(630 \mathrm{mg}, 0.97 \mathrm{mmol}$, 97\%).

${ }^{1} \mathrm{H}$ NMR $\left(500 \mathrm{MHz}, \mathrm{CDCl}_{3}\right) \delta 7.45(\mathrm{dd}, J=8.6,5.5 \mathrm{~Hz}, 2 \mathrm{H}), 7.08(\mathrm{t}, J=8.8 \mathrm{~Hz}$, 2H), 7.03 (ddd, $J=8.8,5.4,1.9 \mathrm{~Hz}, 4 \mathrm{H}), 6.86(\mathrm{dt}, J=12.8,8.7 \mathrm{~Hz}, 4 \mathrm{H}), 0.07(\mathrm{~s}$, 9H), 0.04 (s, 9H), -0.16 (s, 9H) ppm. ${ }^{13} \mathrm{C}$ NMR (126 MHz, CDCl $) \delta 162.7$ (d, J = 246.2 Hz), 162.0 (d, $J=246.3 \mathrm{~Hz}$, 2C), 146.3, 143.4, 142.9, 135.0 (d, J = 3.1 Hz), 135.0 (d, J = 3.8 Hz), 134.9 (d, J=4.1 Hz), 132.2 (d, J = 8.1 Hz), 132.1 (d, $J=8.0 \mathrm{~Hz}$ ), 132.0 (d, J = 8.2 Hz), 126.2, 125.3, 123.5, 114.5 (d, J = 21.2 Hz), 114.4 (d, J = $21.5 \mathrm{~Hz}$ ), 114.3 (d, J = $21.3 \mathrm{~Hz}), 105.3,105.3,104.0,102.7,102.5,102.1,-0.2,-0.3,-0.7$ ppm. ${ }^{19} \mathrm{~F}$ NMR $\left(282 \mathrm{MHz}, \mathrm{CDCl}_{3}\right) \delta-109.2 \mathrm{ppm}$. IR $(\mathbf{A T R}) \tilde{v}=2961,2155,1603,1511,1415,1298,1249,1219,1158,1094,1020,939,913,884,841,761,731$, $702,653,635 \mathrm{~cm}^{-1}$. HRMS $\left(\mathrm{El}^{+}\right) \mathrm{m} / z$ for $\mathrm{C}_{39} \mathrm{H}_{39} \mathrm{~F}_{3} \mathrm{Si}_{3}[\mathrm{M}]^{+}$: calc: 648.2306 , found: 648.2308 . Melting point: 284$287^{\circ} \mathrm{C}$. 


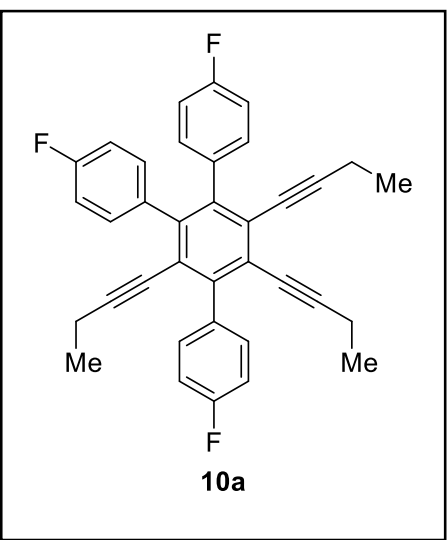

According to General Procedure 4 the title compound was prepared using 1fluoro-4-(hexa-1,3-diyn-1-yl)benzene (344 mg, $2.00 \mathrm{mmol}, 1.00$ equiv.), $\mathrm{CoBr}_{2}$ (dppe) (61.7 mg, $0.10 \mathrm{mmol}, 0.05$ equiv.), zinc (13.1 mg, $0.20 \mathrm{mmol}, 0.10$ equiv.) and zinc(II)iodide ( $63.8 \mathrm{mg}, 0.20 \mathrm{mmol}, 0.10$ equiv.) in $2.00 \mathrm{~mL}$ THF. The product was obtained after filtration over a short plug of silica (eluent: $n$-pentane: $\mathrm{CH}_{2} \mathrm{Cl}_{2}=2: 1$ ) as a colorless solid (341 $\left.\mathrm{mg}, 0.66 \mathrm{mmol}, 99 \%\right)$. The isolated product contains an inseparable mixture of seven regioisomers (2 main isomers).

${ }^{19}$ F NMR $\left(470 \mathrm{MHz}, \mathrm{CDCl}_{3}\right) \delta-113.77,-113.80,-113.83,-113.88,-113.89$, 113.96, -114.02, -114.14, -118.34, -118.52, -118.58, -118.70, -118.76, -118.85, -118.88, -118.92, -118.93, -119.01, -119.09 ppm. IR (ATR) $\tilde{v}=2974,2936,2876,2228,1891,1722,1603,1507,1463,1421,1375,1316,1269,1223$, 1157, 1094, 1064, 1017, 834, 733, $635 \mathrm{~cm}^{-1}$. HRMS $\left(\mathrm{El}^{+}\right) \mathrm{m} / \mathrm{z}$ for $\mathrm{C}_{36} \mathrm{H}_{27} \mathrm{~F}_{3}[\mathrm{M}]^{+}$: calc: 516.2059, found: 516.2067.

\section{4,4'-Difluoro-4'-(4-fluorophenyl)-3',5',6'-tris(3-methylbut-1-yn-1-yl)-1,1':2',1"-terphenyl (10b)}

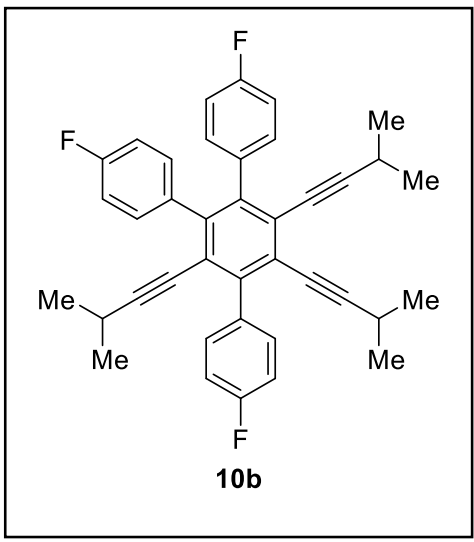

According to General Procedure 4 the title compound was prepared using 1fluoro-4-(5-methylhexa-1,3-diyn-1-yl)benzene $(373 \mathrm{mg}, 2.00 \mathrm{mmol}, 1.00$ equiv.), $\mathrm{CoBr}_{2}$ (dppe) (61.7 mg, $0.10 \mathrm{mmol}, 0.05$ equiv.), zinc (13.1 mg, 0.20 mmol, 0.10 equiv.) and zinc(II)iodide (63.8 mg, $0.20 \mathrm{mmol}, 0.10$ equiv.) in $2.00 \mathrm{~mL}$ THF. The product was obtained after filtration over a short plug of silica (eluent: $n$-pentane: $\mathrm{CH}_{2} \mathrm{Cl}_{2}=2: 1$ ) as a colorless solid (364 mg, $0.65 \mathrm{mmol}$, $98 \%)$. The isolated product contains an inseparable mixture of six regioisomers.

${ }^{19}$ F NMR $\left(470 \mathrm{MHz}, \mathrm{CDCl}_{3}\right) \delta-113.77,-113.81,-113.85,-113.86,-113.91$, $-114.01,-114.18,-118.73,-118.76,-118.91,-118.95,-118.95,-119.02,-119.05,-119.08,-119.21,-119.26$, -119.29 ppm. IR (ATR) $\tilde{v}=2970,2933,2874,2227,1604,1508,1468,1418,1364,1317,1233,1158,1094,1017$, $836,571 \mathrm{~cm}^{-1}$. HRMS $\left(\mathrm{El}^{+}\right) \mathrm{m} / z$ for $\mathrm{C}_{39} \mathrm{H}_{33} \mathrm{~F}_{3}[\mathrm{M}]^{+}$: calc: 558.2529 , found: 558.2532 .

\section{3',4',6'-Tris(3,3-dimethylbut-1-yn-1-yl)-4,4'--difluoro-5'-(4-fluorophenyl)-1,1':2',1"-terphenyl (10c)}

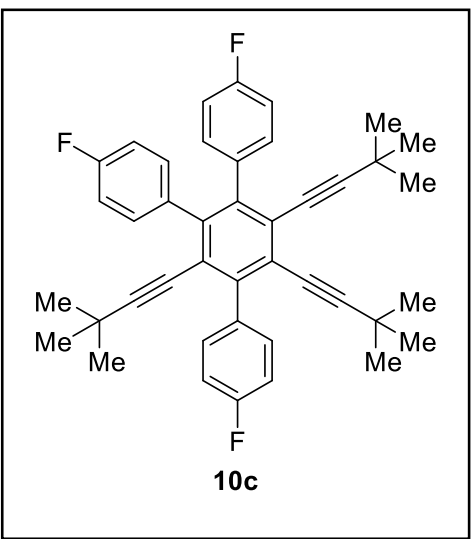

According to General Procedure 4 the title compound was prepared using 1(5,5-dimethylhexa-1,3-diyn-1-yl)-4-fluorobenzene (400 mg, $2.00 \mathrm{mmol}, 1.00$ equiv.), $\mathrm{CoBr}_{2}$ (dppe) (61.7 mg, $0.10 \mathrm{mmol}, 0.05$ equiv.), zinc (13.1 mg, 0.20 mmol, 0.10 equiv.) and zinc(II)iodide (63.8 $\mathrm{mg}, 0.20 \mathrm{mmol}, 0.10$ equiv.) in $2.00 \mathrm{~mL}$ THF. The product was obtained after filtration over a short plug of silica (eluent: $n$-pentane: $\mathrm{CH}_{2} \mathrm{Cl}_{2}=2: 1$ ) as a colorless solid (394 mg, $0.66 \mathrm{mmol}$, 99\%). 
${ }^{1} \mathrm{H}$ NMR $\left(500 \mathrm{MHz}, \mathrm{CDCl}_{3}\right)$ 6 7.43-7.37 (m, 2H), $7.07(\mathrm{t}, J=8.8 \mathrm{~Hz}, 2 \mathrm{H}), 7.02(\mathrm{dt}, J=8.3,5.7 \mathrm{~Hz}, 4 \mathrm{H}), 6.89-6.80(\mathrm{~m}$, $4 \mathrm{H}), 1.10(\mathrm{~s}, 8 \mathrm{H}), 1.07(\mathrm{~s}, 10 \mathrm{H}), 0.80(\mathrm{~s}, 9 \mathrm{H}) \mathrm{ppm}$.

${ }^{13} \mathrm{C}$ NMR $\left(126 \mathrm{MHz}, \mathrm{CDCl}_{3}\right) \delta 162.3(\mathrm{~d}, J=245.2 \mathrm{~Hz}), 161.7$ (d, $\left.J=246.7 \mathrm{~Hz}\right), 161.7$ (d, $\left.J=246.1 \mathrm{~Hz}\right)$ 145.3, 142.3, 141.9, $136.2(\mathrm{~d}, J=3.3 \mathrm{~Hz}), 135.9(\mathrm{~d}, J=3.3 \mathrm{~Hz}), 135.8(\mathrm{~d}, J=3.3 \mathrm{~Hz}), 132.2(\mathrm{~d}, J=8.0 \mathrm{~Hz}), 132.1(\mathrm{~d}, J=7.9 \mathrm{~Hz})$, $131.9(\mathrm{~d}, J=8.1 \mathrm{~Hz}), 125.4,125.3,123.2,114.3(\mathrm{~d}, J=21.7 \mathrm{~Hz}), 114.2(\mathrm{~d}, J=21.4 \mathrm{~Hz}), 114.1(\mathrm{~d}, J=21.7 \mathrm{~Hz}), 107.9$, 107.6, 106.7, 78.1, 77.8, 77.6, 30.7 (2C), 30.2, $28.3(2 \mathrm{C}), 27.9 .{ }^{19} \mathrm{~F}$ NMR (470 MHz, CDCl $)$ $\delta-118.98,-119.13,-$ 119.18 ppm. IR (ATR) $\tilde{v}=2970,2928,2902,2868,2226,1604,1511,1476,1457,1417,1391,1362,1297,1220$, 1159, 1095, 1015, 957, 938, 852, 835, 819, 796, 774, 739, 756, 739, 629, $560 \mathrm{~cm}^{-1}$. HRMS (EI+) $\mathrm{m} / z$ for $\mathrm{C}_{39} \mathrm{H}_{33} \mathrm{~F}_{3}$ $[\mathrm{M}]^{+}$: calc: 558.2529 , found: 558.2532 . Melting point: $334-337^{\circ} \mathrm{C}$ (decomp.)

((4,4"-Dimethoxy-5'-(4-methoxyphenyl)-[1,1':2',1"-terphenyl]-3',4',6'-triyl)tris(ethyne-2,1-diyl))tris(trimethylsilane) (12a)

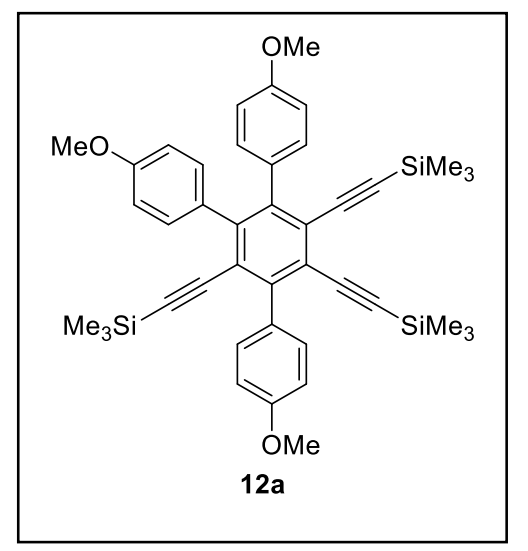

According to General Procedure 4 the title compound was prepared using ((4-methoxyphenyl)buta-1,3-diyn-1-yl)trimethyl-silane $(684 \mathrm{mg}, \quad 3.00$ mmol, 1.00 equiv.), $\mathrm{CoBr}_{2}$ (dppe) (92.6 mg, $0.15 \mathrm{mmol}, 0.05$ equiv.), zinc (19.6 mg, $0.30 \mathrm{mmol}, 0.10$ equiv.) and zinc(II)iodide $(96.0 \mathrm{mg}, 0.30 \mathrm{mmol}$, 0.10 equiv.) in $3.00 \mathrm{~mL}$ THF. The product was obtained after filtration over a short plug of silica (eluent: $n$-pentane: $\mathrm{CH}_{2} \mathrm{Cl}_{2}=1: 1$ ) as a beige solid (647 $\mathrm{mg}, 0.95 \mathrm{mmol}, 95 \%)$.

${ }^{1} \mathbf{H}$ NMR $\left(300 \mathrm{MHz}^{\left.-\mathrm{CDCl}_{3}\right)}\right.$ 8 7.46-7.41 (m, 2H), 7.03-6.97 (m, 4H), 6.94-6.88 $(\mathrm{m}, 2 \mathrm{H}), 6.72-6.64(\mathrm{~m}, 4 \mathrm{H}), 3.85(\mathrm{~s}, 3 \mathrm{H}), 3.75(\mathrm{~s}, 3 \mathrm{H}), 3.73(\mathrm{~s}, 3 \mathrm{H}), 0.07(\mathrm{~s}$,

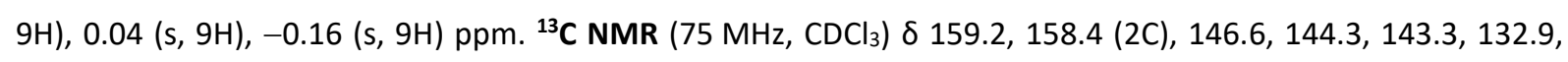
$131.8,131.7$ (2C), 131.6, 126.2, 124.7, 123.6, 114.1, 112.8, 112.7, 112.7, 104.1, 104.0, 103.5, 103.4, 102.9, 102.8, 55.5, 55.3 (2C), $-0.1,-0.2,-0.5$ ppm. IR (ATR) $\tilde{v}=2955,2957,2899,2835,2152,1608,1577,1515,1464,1442$, 1417, 1404, 1383, 1288, 1243, 1174, 1109, 1036, 1006, 936, 913, 883, 838, 759, 699, 657, $639 \mathrm{~cm}^{-1}$. HRMS (El+) $\mathrm{m} / \mathrm{z}$ for $\mathrm{C}_{42} \mathrm{H}_{48} \mathrm{O}_{3} \mathrm{Si}_{3}[\mathrm{M}]^{+}$: calc: 684.2906 , found: 684.2904 . Melting point: $238-241{ }^{\circ} \mathrm{C}$.

((3,3'-Dimethoxy-5'-(3-methoxyphenyl)-[1,1':2',1"'-terphenyl]-3',4',6'-triyl)tris(ethyne-2,1-diyl))tris(trimethylsilane) (12b)

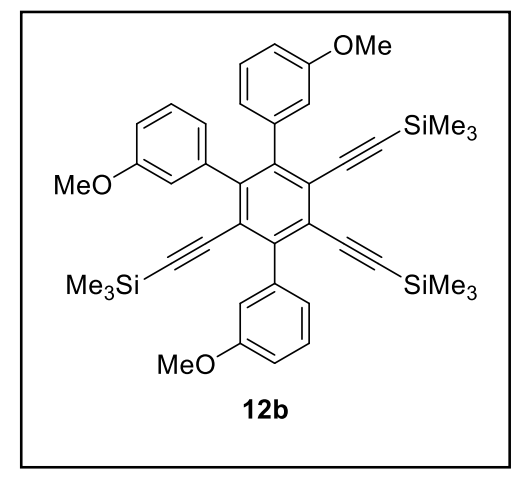

According to General Procedure 4 the title compound was prepared using ((3-methoxyphenyl)buta-1,3-diyn-1-yl)trimethyl-silane $(684 \mathrm{mg}, 3.00$ mmol, 1.00 equiv.), $\mathrm{CoBr}_{2}$ (dppe) (92.6 mg, $0.15 \mathrm{mmol}, 0.05$ equiv.), zinc (19.6 mg, $0.30 \mathrm{mmol}, 0.10$ equiv.) and zinc(II)iodide $(96.0 \mathrm{mg}, 0.30 \mathrm{mmol}$, 0.10 equiv.) in $3.00 \mathrm{~mL}$ THF. The product was obtained after filtration over a short plug of silica (eluent: $n$-pentane: $\mathrm{CH}_{2} \mathrm{Cl}_{2}=1: 1$ ) as a brown glassy solid (592 mg, $0.87 \mathrm{mmol}, 87 \%$ ).

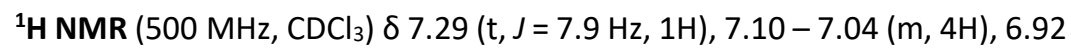
(ddd, $J=8.3,2.7,1.0 \mathrm{~Hz}, 1 \mathrm{H}), 6.74-6.66(\mathrm{~m}, 6 \mathrm{H}), 3.84(\mathrm{~s}, 3 \mathrm{H}), 3.66(\mathrm{~s}, 3 \mathrm{H}), 3.65(\mathrm{~s}, 3 \mathrm{H}), 0.07(\mathrm{~s}, 9 \mathrm{H}), 0.04(\mathrm{~s}, 9 \mathrm{H})$, -0.17 (s, 9H).ppm. ${ }^{13} \mathrm{C}$ NMR (126 MHz, $\mathrm{CDCl}_{3}$ ) $\delta 158.9,158.8,158.7,147.2,144.1$ (2C), 143.5, 140.5, 140.4, 128.4, 
128.3, 128.2, 125.8, 124.9, 123.2, 123.1, 123.0, 122.7, 115.9, 115.9, 115.4, 113.9, 113.2, 113.1, 104.6, 104.6, 103.3, 103.0, 102.9, 102.5, 55.4, 55.3, 55.3, -0.2, -0.3, -0.6 ppm. IR (ATR) $\tilde{v}=2959,2902,2835,2156,1602$, $1581,1491,1465,1430,1386,1318,1286,1249,1163,1139,1082,1048,997,963,925,839,781,759,738,699$, $651,638,569 \mathrm{~cm}^{-1}$. HRMS $\left(\mathrm{El}^{+}\right) \mathrm{m} / z$ for $\mathrm{C}_{42} \mathrm{H}_{48} \mathrm{O}_{3} \mathrm{Si}_{3}[\mathrm{M}]^{+}$: calc: 684.2910 , found: 684.2906 .

((2,2"-Dimethoxy-5'-(2-methoxyphenyl)-[1,1':2',1"-terphenyl]-3',4',6'-triyl)tris(ethyne-2,1-diyl))tris(trimethylsilane) (12c)

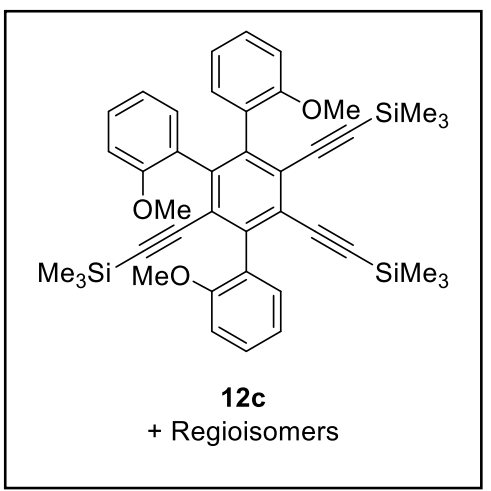

According to General Procedure 4 the title compound was prepared using ((2-methoxyphenyl)buta-1,3-diyn-1-yl)trimethyl-silane $(684 \mathrm{mg}, 3.00 \mathrm{mmol}$, 1.00 equiv.), $\mathrm{CoBr}_{2}$ (dppe) (92.6 mg, $0.15 \mathrm{mmol}, 0.05$ equiv.), zinc (19.6 mg, $0.30 \mathrm{mmol}, 0.10$ equiv.) and zinc(II)iodide ( $96.0 \mathrm{mg}, 0.30 \mathrm{mmol}, 0.10$ equiv.) in $3.00 \mathrm{~mL}$ THF. The products were obtained after filtration over a short plug of silica (eluent: $n$-pentane: $\mathrm{CH}_{2} \mathrm{Cl}_{2}$ ) an orange solid $(612 \mathrm{mg}, 0.89 \mathrm{mmol}$, $89 \%)$. The product consisted of more than one regioisomer.

IR (ATR) $\tilde{v}=2958,2898,2834,2154,1601,1581,1493,1461,1433,1392$, 1246, 1180, 1161, 1113, 1091, 1048, 1028, 914, 885, 838, 785, 749, 698, 647, $557 \mathrm{~cm}^{-1}$. HRMS (EI+) $\mathrm{m} / \mathrm{z}$ for $\mathrm{C}_{42} \mathrm{H}_{48} \mathrm{O}_{3} \mathrm{Si}_{3}[\mathrm{M}]^{+}$: calc: 684.2909 , found: 684.2906 . Melting point: $73-76^{\circ} \mathrm{C}$

((2,2"'-Dimethoxy-5'-(2-methoxyphenyl)-[1,1':2',1"-terphenyl]-3',4',6'-triyl)tris(ethyne-2,1-diyl))tris(triisopropylsilane) (12d)

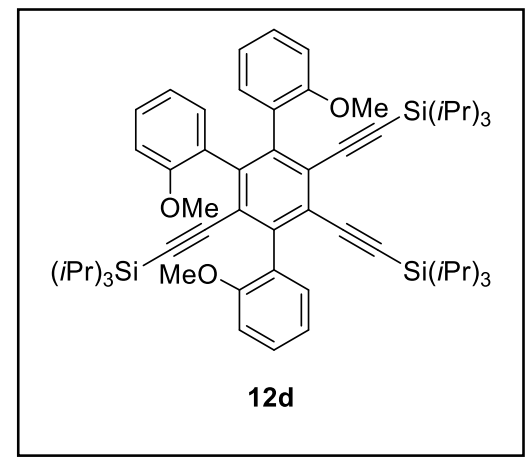

According to General Procedure 4 the title compound was prepared using ((2-methoxyphenyl)buta-1,3-diyn-1-yl)triisopropylsilane (313 mg, 1.00 mmol, 1.00 equiv.), $\mathrm{CoBr}_{2}$ (dppe) (30.9 mg, $0.05 \mathrm{mmol}, 0.05$ equiv.), zinc (6.6 mg, $0.10 \mathrm{mmol}, 0.10$ equiv.) and zinc(II)iodide (32.0 mg, $0.10 \mathrm{mmol}$, 0.10 equiv.) in $1.00 \mathrm{~mL}$ THF. The product was obtained after column chromatography (eluent: $n$-pentane: $\mathrm{CH}_{2} \mathrm{Cl}_{2} 3: 1$ ) an orange solid (52.0 mg, $0.06 \mathrm{mmol}, 17 \%$, total yield of isomers: $78 \%$ ).

${ }^{1} \mathrm{H}$ NMR $\left(500 \mathrm{MHz}, \mathrm{CDCl}_{3}\right) \delta 7.20(\mathrm{dd}, J=8.2,6.7 \mathrm{~Hz}, 2 \mathrm{H}), 7.09-7.02(\mathrm{~m}$, 2H), $6.99(\mathrm{td}, J=7.8,1.8 \mathrm{~Hz}, 1 \mathrm{H}), 6.91(\mathrm{t}, J=7.4 \mathrm{~Hz}, 1 \mathrm{H}), 6.87(\mathrm{~d}, J=8.2 \mathrm{~Hz}, 1 \mathrm{H}), 6.77-6.72(\mathrm{~m}, 2 \mathrm{H}), 6.65(\mathrm{~d}, J=8.2$ $\mathrm{Hz}, 1 \mathrm{H}), 6.57(\mathrm{t}, J=8.2 \mathrm{~Hz}, 2 \mathrm{H}), 3.77(\mathrm{~s}, 3 \mathrm{H}), 3.65(\mathrm{~s}, 3 \mathrm{H}), 3.48(\mathrm{~s}, 3 \mathrm{H}), 0.96-0.80(\mathrm{~m}, 42 \mathrm{H}), 0.73-0.60(\mathrm{~m}, 21 \mathrm{H})$ ppm. ${ }^{13} \mathrm{C}$ NMR $\left(126 \mathrm{MHz}, \mathrm{CDCl}_{3}\right) \delta$ 157.4, 157.3, 156.9, 145.1, 141.8, 141.7, 132.5, 131.5, 131.3, 130.0, 129.0, $128.8,128.7,128.3,128.2,125.8,125.3$ (2C), 120.6, 119.5, 119.2, 111.3, 110.2, 110.1, 105.4, 105.3, 104.6, 99.3, 98.2, 97.0, 55.6, 55.3, 54.9, $18.8(12 \mathrm{C})^{*}, 18.5$ (6C) , 11.5 (2C), 11.1 ppm.. IR (ATR) $\tilde{v}=3048,2941,2891,2863$, $2146,1957,1602,1581,1495,1461,1434,1390,1274,1247,1182,1159,1113,1051,1028,995,913,881,821$, 788, 743, $675 \mathrm{~cm}^{-1}$. HRMS $\left(\mathrm{EI}^{+}\right) \mathrm{m} / z$ for $\mathrm{C}_{42} \mathrm{H}_{48} \mathrm{O}_{3} \mathrm{Si}_{3}[\mathrm{M}]^{+}$: calc: 684.2909 , found: 684.2906 . Melting point: 156$159^{\circ} \mathrm{C}$.

*The signal of the isopropyl group shows a multiplicity in the NMR for an unknown reason. 


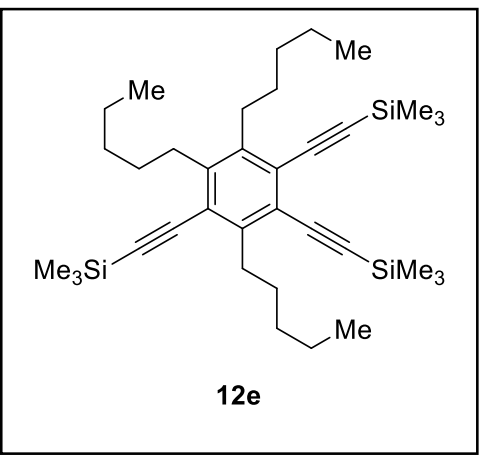

According to General Procedure 4 the title compound was prepared using trimethyl(nona-1,3-diyn-1-yl)silane (577 mg, $3.00 \mathrm{mmol}, 1.00$ equiv.), $\mathrm{CoBr}_{2}$ (dppe) (92.6 mg, $0.15 \mathrm{mmol}, 0.05$ equiv.), zinc (19.6 mg, $0.30 \mathrm{mmol}$, 0.10 equiv.) and zinc(II)iodide ( $96.0 \mathrm{mg}, 0.30 \mathrm{mmol}, 0.10$ equiv.) in $3.00 \mathrm{~mL}$ THF. The product was obtained after filtration over a short plug of silica (eluent: $n$-pentane: $\mathrm{CH}_{2} \mathrm{Cl}_{2}=10: 1$ ) as yellow-orange oil $(523 \mathrm{mg}, 0.91 \mathrm{mmol}$, 91\%).

${ }^{1} \mathrm{H}$ NMR $\left(300 \mathrm{MHz}, \mathrm{CDCl}_{3}\right) \delta$ 2.96-2.92 (m, 2H), 2.76 (ddd, $J=16.5,8.2,5.2$

$\mathrm{Hz}, 4 \mathrm{H}), 1.63-1.56(\mathrm{~m}, 2 \mathrm{H}), 1.55-1.47(\mathrm{~m}, 4 \mathrm{H}), 1.37(\mathrm{tt}, J=8.6,4.9 \mathrm{~Hz}, 12 \mathrm{H}), 0.94-0.89(\mathrm{~m}, 9 \mathrm{H}), 0.27(\mathrm{~s}, 9 \mathrm{H}), 0.26$

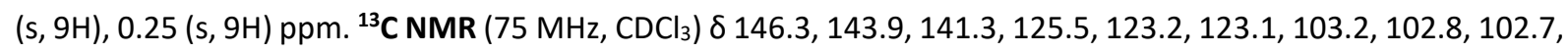
102.6, 102.5, 101.2, 34.1, 32.6 (3C), 32.2, 31.9, 30.3, 30.2, 29.3, 22.7 (3C), 14.3 (3C), 0.2 (2C), 0.1 ppm. IR (ATR) $\tilde{v}=2597,2928,2861,2150,1462,1411,1378,1248,1114,988,904,838,758,699,641,582 \mathrm{~cm}^{-1}$. HRMS $\left(\mathrm{EI}^{+}\right)$ $m / z$ for $\mathrm{C}_{42} \mathrm{H}_{48} \mathrm{O}_{3} \mathrm{Si}_{3}[\mathrm{M}]^{+}$: calc: 684.2909 , found: 684.2906 .

\section{Cobalt-Catalyzed Cyclotrimerization of Symmetrical 1,3-Butadiynes}

\section{4'-Phenyl-3',5',6'-tris(phenylethynyl)-1,1':2',1'-terphenyl (14a)}

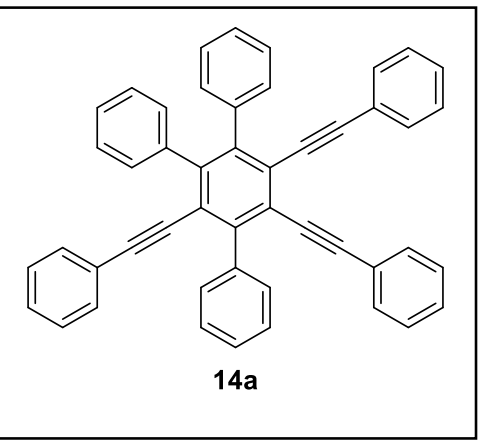

According to General Procedure 4 the title compound was prepared using 1,4-diphenylbutadiyne (606 mg, 3.00 mmol, 1.00 equiv.), $\mathrm{CoBr}_{2}$ (dppe) (92.6 $\mathrm{mg}, 0.15 \mathrm{mmol}, 0.05$ equiv.), zinc (19.6 mg, $0.30 \mathrm{mmol}, 0.10$ equiv.) and zinc(II)iodide ( $96.0 \mathrm{mg}, 0.30 \mathrm{mmol}, 0.10$ equiv.) in $3.00 \mathrm{~mL}$ THF. The product was obtained after filtration over a short plug of silica (eluent: $\mathrm{Et}_{2} \mathrm{O}$ ) as a light brown solid (605 mg, $0.99 \mathrm{mmol}, 99 \%)$.

${ }^{1} \mathbf{H}$ NMR $\left(300 \mathrm{MHz}, \mathrm{CDCl}_{3}\right) \delta 7.72(\mathrm{~d}, J=7.2 \mathrm{~Hz}, 2 \mathrm{H}), 7.53(\mathrm{dd}, J=10.1,6.9$ $\mathrm{Hz}, 4 \mathrm{H}), 7.31-7.06(\mathrm{~m}, 30 \mathrm{H}), 6.75(\mathrm{~d}, J=7.2 \mathrm{~Hz}, 2 \mathrm{H}) \mathrm{ppm} .{ }^{13} \mathrm{C}$ NMR $(75 \mathrm{MHz}$, $\left.\mathrm{CDCl}_{3}\right) \delta 146.1,143.7,143.2,139.6,139.5,139.4,131.6$ (2C), 131.3, 130.8, 130.8, 130.6, 128.5 (2C), 128.4, 128.3, 128.2, 128.1, 127.8, 127.6, 127.5, 127.4, 127.0 (2C), 125.7, 124.8, 123.5, 123.5, 123.3, 123.2, 98.6, 98.4, 97.4, 89.0, 88.9, 88.3 ppm. IR (ATR) $\tilde{v}=3078,3056,3031,2964,2926,2340,2208,2452,1949,1882,1804,1756$, $1673,1597,1571,1492,1442,1404,1329,1262,1238,1176,1157,1097,1070,1026,1003,915,874,841,805$, 754, 716, 689, 619, 607, 586, $573 \mathrm{~cm}^{-1}$. HRMS $\left(\mathrm{EI}^{+}\right) \mathrm{m} / 2$ for $\mathrm{C}_{48} \mathrm{H}_{30}[\mathrm{M}]^{+}$: calc: 606.2342 , found: 606.2338 . Melting point: $228-232^{\circ} \mathrm{C}$. 


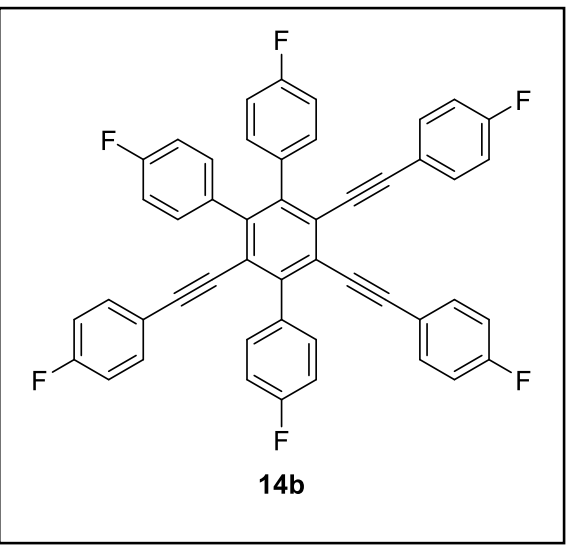

According to General Procedure 4 the title compound was prepared using 1,4-bis(4-fluorophenyl)butadiyne $(715 \mathrm{mg}, 3.00 \mathrm{mmol}, 1.00$ equiv.), $\mathrm{CoBr}_{2}$ (dppe) (92.6 mg, $0.15 \mathrm{mmol}, 0.05$ equiv.), zinc (19.6 mg, $0.30 \mathrm{mmol}, 0.10$ equiv.) and zinc(II)iodide $(96.0 \mathrm{mg}, 0.30 \mathrm{mmol}, 0.10$ equiv.) in $3.00 \mathrm{~mL}$ THF. The product was obtained after filtration over a short plug of silica (eluent: $\mathrm{Et}_{2} \mathrm{O}$ ) as a light brown solid ( $685 \mathrm{mg}, 0.96$ mmol, 96\%).

${ }^{1} \mathrm{H}$ NMR $\left(500 \mathrm{MHz}, \mathrm{CDCl}_{3}\right) 7.65(\mathrm{dd}, J=8.5,5.6 \mathrm{~Hz}, 2 \mathrm{H}), 7.29-7.12(\mathrm{~m}$, $12 \mathrm{H}), 7.02-6.92(\mathrm{~m}, 9 \mathrm{H}), 6.88(\mathrm{t}, J=8.7 \mathrm{~Hz}, 2 \mathrm{H}), 6.76(\mathrm{dd}, J=8.7,5.5$

$\mathrm{Hz}, 2 \mathrm{H})$ ppm. ${ }^{13} \mathrm{C}$ NMR $\left(126 \mathrm{MHz}, \mathrm{CDCl}_{3}\right) \delta 162.9(\mathrm{~d}, J=250.8 \mathrm{~Hz}, 2 \mathrm{C}), 162.8(\mathrm{~d}, J=247.2 \mathrm{~Hz}), 162.7(\mathrm{~d}, J=250.5$ $\mathrm{Hz}), 162.1(\mathrm{~d}, J=247.0 \mathrm{~Hz}, 2 \mathrm{C}), 145.0,142.8,142.4,135.4(\mathrm{~d}, J=3.2 \mathrm{~Hz}), 135.2(\mathrm{~d}, J=3.5 \mathrm{~Hz}), 135.0(\mathrm{~d}, J=3.3 \mathrm{~Hz})$, $133.4(\mathrm{~d}, J=8.5 \mathrm{~Hz}, 2 \mathrm{C}), 133.14(\mathrm{~d}, J=8.4 \mathrm{~Hz}), 132.4(\mathrm{~d}, J=7.6 \mathrm{~Hz}), 132.3(\mathrm{~d}, J=7.4 \mathrm{~Hz}), 132.3(\mathrm{~d}, J=7.7 \mathrm{~Hz}), 125.8$, 125.2, 123.5, 119.3 (d, $J=3.3 \mathrm{~Hz}$ ), 119.2 (d, $J=3.6 \mathrm{~Hz}$ ), 118.9 (d, $J=3.3 \mathrm{~Hz}), 115.9$ (d, $J=21.5 \mathrm{~Hz}, 2 \mathrm{C}), 115.8(\mathrm{~d}, J$ $=22.0 \mathrm{~Hz}$ ), $114.7(\mathrm{~d}, J=21.5 \mathrm{~Hz}, 2 \mathrm{C}), 114.6(\mathrm{~d}, J=21.5 \mathrm{~Hz}), 98.1,98.0,96.9,88.2,88.0,87.5{ }^{19} \mathrm{~F} \mathrm{NMR}(470 \mathrm{MHz}$, $\left.\mathrm{C}_{6} \mathrm{~F}_{6}\right) \delta-112.9,-113.0,-113.1,-117.0,-117.7$ (2F) ppm. IR (ATR) $\tilde{v}=2213,1893,1601,1504,1418,1297,1230$, 1154, 1093, 1015, 908, 831, 772, 733, 672, 6460, $570 \mathrm{~cm}^{-1}$. HRMS (El+) m/z for $\mathrm{C}_{48} \mathrm{H}_{30}[\mathrm{M}]^{+}$: calc: 714.1782 , found: 714.1780. Melting point: $240-243^{\circ} \mathrm{C}$.

\section{4,4"-Dichloro-4'-(4-chlorophenyl)-3',5',6'-tris((4-chlorophenyl)ethynyl)-1,1':2',1"-terphenyl (14c)}

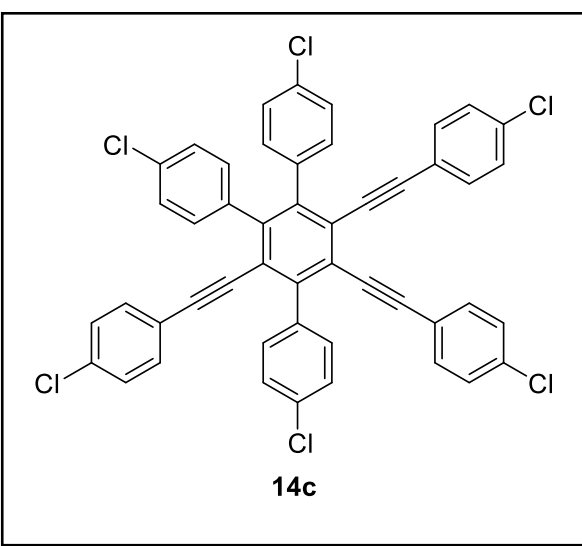

According to General Procedure 4 the title compound was prepared using 1,4-bis(4-chlorophenyl)butadiyne $(271 \mathrm{mg}, 1.00 \mathrm{mmol}, 1.00$ equiv.), $\mathrm{CoBr}_{2}$ (dppe) (31.0 mg, $0.05 \mathrm{mmol}, 0.05$ equiv.), zinc (6.60 $\mathrm{mg}, 0.10 \mathrm{mmol}, 0.10$ equiv.) and zinc(II)iodide $(32.0 \mathrm{mg}, 0.10 \mathrm{mmol}$, 0.10 equiv.) in $2.00 \mathrm{~mL}$ THF. The product was obtained after filtration over a short plug of silica (eluent: $n$-pentane: $\mathrm{CH}_{2} \mathrm{Cl}_{2}=3: 1$ ) as a light orange solid (251 mg, $0.31 \mathrm{mmol}, 93 \%$ ).

${ }^{1} \mathrm{H}$ NMR $\left(500 \mathrm{MHz}, \mathrm{CDCl}_{3}\right) \delta 7.69(\mathrm{~d}, J=8.4 \mathrm{~Hz}, 2 \mathrm{H}), 7.62(\mathrm{~d}, J=8.4$ $\mathrm{Hz}, 2 \mathrm{H}), 7.40-7.33(\mathrm{~m}, 8 \mathrm{H}), 7.29-7.22(\mathrm{~m}, 8 \mathrm{H}), 7.18(\mathrm{~d}, J=8.5 \mathrm{~Hz}$,

$2 \mathrm{H}), 6.78(\mathrm{~d}, J=8.4 \mathrm{~Hz}, 2 \mathrm{H}) \mathrm{ppm} .{ }^{13} \mathrm{C}$ NMR $\left(126 \mathrm{MHz}, \mathrm{CDCl}_{3}\right) \delta 145.0,142.7,142.3,137.6,137.4,137.2,135.1$ (2C), $134.9,134.2,133.6$ (2C), 132.7 (2C), 132.4, 132.0 (2C), 131.9, 129.0 (2C), 128.8, 128.1, 128.0 (2C), 125.9, 125.2, 123.3, 121.4 (2C), 121.1, 98.4 (2C), 97.1, 89.2, 88.9, 88.5 ppm. IR (ATR) $\tilde{v}=3569,3315,2363,2338,2213,2167$, 1594, 1489, 1418, 1392, 1265, 1177, 1091, 1015, 824, 766, 748, 704, $636 \mathrm{~cm}^{-1}$. HRMS (El+) m/z for $\mathrm{C}_{48} \mathrm{H}_{24} \mathrm{Cl}_{6}\left[\mathrm{M}^{+}\right.$: calc: 810.0004 , found: 809.9990 . Melting point: $288-291^{\circ} \mathrm{C}$ (decomp.) 


\section{4,4"-Dibromo-4'-(4-bromophenyl)-3',5',6'-tris((4-bromophenyl)ethynyl)-1,1':2',1"-terphenyl (14d)}

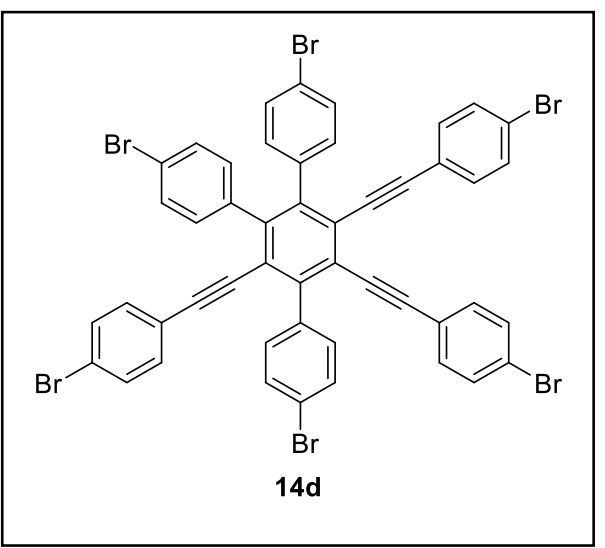

According to General Procedure 4 the title compound was prepared using 1,4-bis(4-bromophenyl)butadiyne (360 mg, $1.00 \mathrm{mmol}, 1.00$ equiv.), $\mathrm{CoBr}_{2}$ (dppe) (31.0 mg, $0.05 \mathrm{mmol}, 0.05$ equiv.), zinc (6.60 $\mathrm{mg}, 0.10 \mathrm{mmol}, 0.10$ equiv.) and zinc(II)iodide $(32.0 \mathrm{mg}, 0.10 \mathrm{mmol}$, 0.10 equiv.) in $2.00 \mathrm{~mL}$ THF. The product was obtained after filtration over a short plug of silica (eluent: $\mathrm{Et}_{2} \mathrm{O}$ ) as a light brown solid (358 mg, $0.33 \mathrm{mmol}, 99 \%)$.

${ }^{1} \mathrm{H}$ NMR $\left(500 \mathrm{MHz}, \mathrm{CDCl}_{3}\right) 7.66(\mathrm{~d}, J=8.3 \mathrm{~Hz}, 2 \mathrm{H}), 7.51(\mathrm{~d}, J=8.4 \mathrm{~Hz}$, $2 \mathrm{H}), 7.46-7.37(\mathrm{~m}, 8 \mathrm{H}), 7.33(\mathrm{~d}, J=8.5 \mathrm{~Hz}, 2 \mathrm{H}), 7.07(\mathrm{t}, J=8.2 \mathrm{~Hz}$, $6 \mathrm{H}), 6.99$ (d, $J=8.4 \mathrm{~Hz}, 2 \mathrm{H}), 6.60$ (d, $J=8.5 \mathrm{~Hz}, 2 \mathrm{H})$ ppm. ${ }^{13} \mathrm{C} \mathrm{NMR}\left(126 \mathrm{MHz}, \mathrm{CDCl}_{3}\right) \delta$ 145.1, 142.6, 142.2, 138.0, $137.8,137.6,132.9$ (2C), 132.6, 132.3, 132.2, 132.1, 131.9 (2C), 131.8, 131.1, 131.0 (2C), 125.8, 125.1, 123.4 (2C), 123.2 (2C), 122.4, 121.9, 121.8 (3C), 121.5, 98.6 (2C), 97.3, 89.3, 89.1, 88.6 ppm. IR (ATR) $\tilde{v}=3317,3217,3114$, 2967, 2926, 2710, 2545, 2435, 2357, 2216, 2063, 1996, 1891, 1640, 1588, 1486, 1415, 1389, 1262, 1177, 1070, 1011, 821, 755, 734, $646 \mathrm{~cm}^{-1}$. HRMS $\left(\mathrm{El}^{+}\right) \mathrm{m} / \mathrm{z}$ for $\mathrm{C}_{48} \mathrm{H}_{24} \mathrm{Br}_{6}[\mathrm{M}]^{+}$: calc: 1073.6973 , found: 1073.6973. Melting point: $297-300^{\circ} \mathrm{C}$ (decomp).

\section{4,4"-Dimethoxy-4'-(4-methoxyphenyl)-3',5',6'-tris((4-methoxyphenyl)ethynyl)-1,1':2',1"'-terphenyl (14e)}

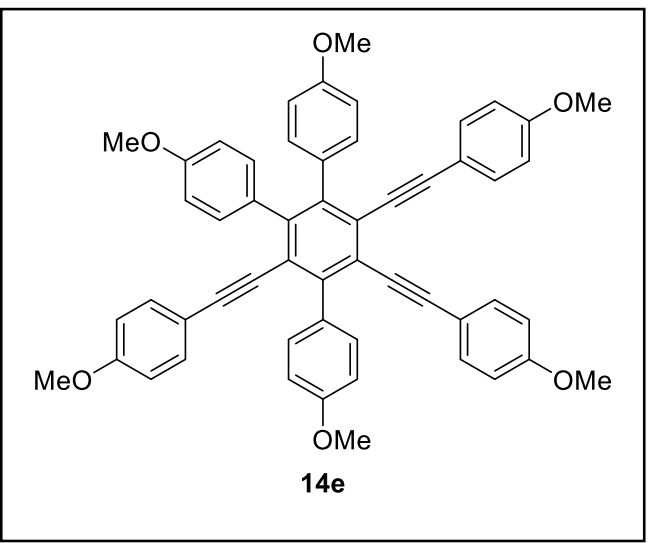

According to General Procedure 4 the title compound was prepared using 1,4-bis(4-methoxyphenyl)buta-1,3-diyne (787 $\mathrm{mg}, 3.00 \mathrm{mmol}, 1.00$ equiv.), [CoBr 2 (dppe)] (92.6 mg, $0.15 \mathrm{mmol}$, 0.05 equiv.), zinc (19.6 $\mathrm{mg}, 0.30 \mathrm{mmol}, 0.10$ equiv.) and zinc(II)iodide ( $96.0 \mathrm{mg}, 0.30 \mathrm{mmol}, 0.10$ equiv.) in $3.00 \mathrm{~mL}$ THF. The product was obtained after column chromatography $(n-$ pentane: $\mathrm{CH}_{2} \mathrm{Cl}_{2}=1: 1$ ) as an off-white solid (570 $\mathrm{mg}, 0.72 \mathrm{mmol}$, $72 \%)$.

${ }^{1} \mathbf{H}$ NMR $\left(500 \mathrm{MHz}, \mathrm{CDCl}_{3}\right) \delta 7.64(\mathrm{~d}, J=8.5 \mathrm{~Hz}, 2 \mathrm{H}), 7.23(\mathrm{~d}, J=$ $8.5 \mathrm{~Hz}, 2 \mathrm{H}), 7.15(\mathrm{t}, J=8.7 \mathrm{~Hz}, 6 \mathrm{H}), 7.05(\mathrm{~d}, J=8.5 \mathrm{~Hz}, 2 \mathrm{H}), 6.82-6.74(\mathrm{~m}, 10 \mathrm{H}), 6.67(\mathrm{~d}, J=8.7 \mathrm{~Hz}, 2 \mathrm{H}), 3.92(\mathrm{~s}$, $3 \mathrm{H}), 3.80(\mathrm{~s}, 3 \mathrm{H}), 3.79$ (s, 3H), 3.78 (s, 6H, 2x OMe), $3.74(\mathrm{~s}, 3 \mathrm{H})$ ppm. ${ }^{13} \mathrm{C} \mathrm{NMR}\left(126 \mathrm{MHz}, \mathrm{CDCl}_{3}\right) \delta 159.8(2 \mathrm{C})$, 159.6, 159.2, 158.4 (2C), 144.8, 143.0, 142.5, 133.1 (2C), 132.8, 132.4, 132.4, 132.2, 132.1, 132.1, 132.0, 125.8, 124.9, 123.6, 116.1, 116.0, 115.8, 114.1 (2C), 113.9, 112.9 (2C), 112.8, 98.3, 98.1, 97.1, 88.4 (2C), 87.7, 55.6, 55.4, 55.4, 55.4, 55.3 (2C) ppm. IR (ATR) $\tilde{v}=3006,2961,2933,2836,2541,2202,1603,1567,1507,1462,1439,1417$, $1353,1289,1245,1168,1105,1025,933,828,803,774,731,683,667,647,598 \mathrm{~cm}^{-1}$.

HRMS $\left(\mathrm{EI}^{+}\right) \mathrm{m} / \mathrm{z}$ for $\mathrm{C}_{54} \mathrm{H}_{42} \mathrm{O}_{6}[\mathrm{M}]^{+}$: calc: 586.2976 , found: 586.2989 . Melting point: $246-249{ }^{\circ} \mathrm{C}$. 


\section{4,4"-Dimethyl-4'-(p-tolyl)-3',5',6'-tris(p-tolylethynyl)-1,1':2',1"-terphenyl (14f)}

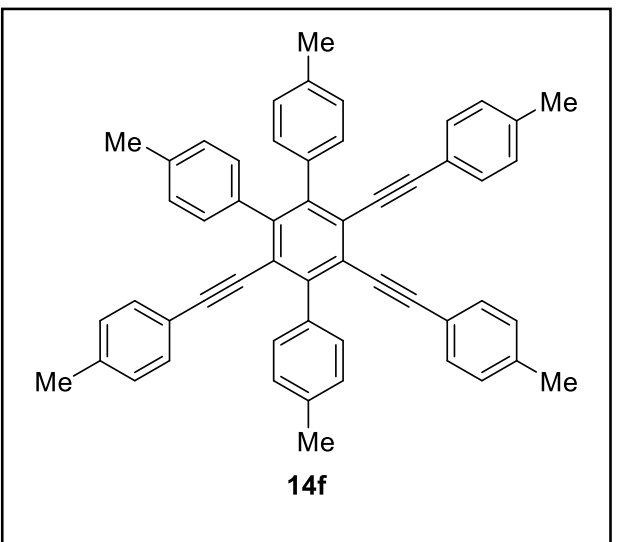

According to General Procedure 4 the title compound was prepared using 1,4-di-p-tolylbutadiyne $(691 \mathrm{mg}, 3.00 \mathrm{mmol}, 1.00$ equiv.), $\mathrm{CoBr}_{2}$ (dppe) (92.6 mg, $0.15 \mathrm{mmol}, 0.05$ equiv.), zinc (19.6 $\mathrm{mg}, 0.30 \mathrm{mmol}, 0.10$ equiv.) and zinc(II)iodide $(96.0 \mathrm{mg}, 0.30$ $\mathrm{mmol}, 0.10$ equiv.) in $3.00 \mathrm{~mL}$ THF. The product was obtained after filtration over a short plug of silica (eluent: $n$-pentane: $\mathrm{CH}_{2} \mathrm{Cl}_{2}=3: 1$ ) as a light beige solid (683 $\mathrm{mg}, 0.99 \mathrm{mmol}, 99 \%$ ).

${ }^{1} \mathrm{H}$ NMR $\left(500 \mathrm{MHz}, \mathrm{CDCl}_{3}\right) \delta 7.60(\mathrm{~d}, J=8.0 \mathrm{~Hz}, 2 \mathrm{H}), 7.32(\mathrm{~d}, J=7.8$ $\mathrm{Hz}, 2 \mathrm{H}), 7.18(\mathrm{~d}, J=8.0 \mathrm{~Hz}, 2 \mathrm{H}), 7.14(\mathrm{dd}, J=9.3,7.9 \mathrm{~Hz}, 4 \mathrm{H}), 7.10-$

$7.02(\mathrm{~m}, 10 \mathrm{H}), 6.94(\mathrm{~d}, J=7.9 \mathrm{~Hz}, 2 \mathrm{H}), 6.67(\mathrm{~d}, J=8.0 \mathrm{~Hz}, 2 \mathrm{H}), 2.49(\mathrm{~s}, 3 \mathrm{H}), 2.34(\mathrm{~s}, 3 \mathrm{H}), 2.34(\mathrm{~s}, 3 \mathrm{H}), 2.32(\mathrm{~s}, 6 \mathrm{H})$, 2.27 (s, 3H) ppm. ${ }^{13} \mathrm{C}$ NMR $\left(126 \mathrm{MHz} \mathrm{CDCl}_{3}\right) \delta 145.6,143.6,143.0,138.4(2 \mathrm{C}), 138.1,137.1,136.9,136.8,136.7$, 136.2 (2C), 131.5 (2C), 131.2, 130.8, 130.7, 130.6, 129.1 (2C), 128.9, 128.1 (2C), 128.0, 125.9, 124.8, 123.5, 120.8 (2C), 120.6, 98.5, 98.3, 97.3, 88.9 (2C), 88.2, 21.6 (2C), 21.6 (2C), 21.4 (2C) ppm. IR (ATR) $\tilde{v}=2918,2210,2111$, 1901, 1511, 1414, 1246, 1180, 1111, 1022, 838, 813, 738, 641, $594 \mathrm{~cm}^{-1}$. HRMS (El+) m/z for C48H HM] $_{30}^{+}$: calc: 690.3286, found: 690.3281 . Melting point: $273-276^{\circ} \mathrm{C}$.

\section{3,3"-Dimethyl-4'-(m-tolyl)-3',5',6'-tris(m-tolylethynyl)-1,1':2',1"-terphenyl (14h)}

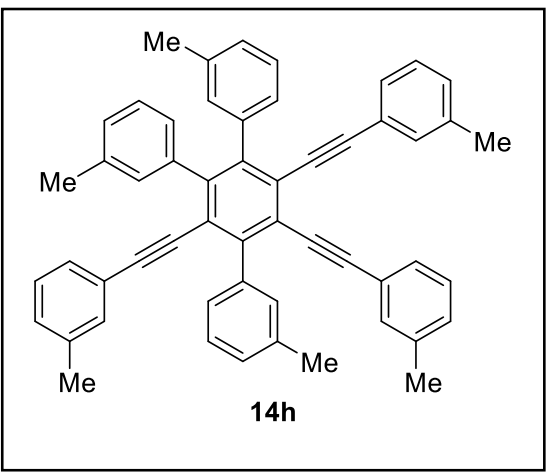

According to General Procedure 4 the title compound was prepared using 1,4-di-m-tolylbutadiyne (691 $\mathrm{mg}, 3.00 \mathrm{mmol}, 1.00$ equiv.), $\mathrm{CoBr}_{2}$ (dppe) (92.6 mg, $0.15 \mathrm{mmol}, 0.05$ equiv.), zinc (19.6 mg, 0.30 mmol, 0.10 equiv.) and zinc(II)iodide (96.0 mg, $0.30 \mathrm{mmol}, 0.10$ equiv.) in $3.00 \mathrm{~mL}$ THF. The product was obtained after filtration over a short plug of silica (eluent: $n$-pentan: $\mathrm{CH}_{2} \mathrm{Cl}_{2}=3: 1$ ) as an orange solid $(657 \mathrm{mg}$, $0.95 \mathrm{mmol}, 95 \%)$.

${ }^{1} \mathrm{H}$ NMR $\left(500 \mathrm{MHz}, \mathrm{CDCl}_{3}\right) \delta 7.55(\mathrm{~s}, 1 \mathrm{H}), 7.53(\mathrm{~d}, J=7.8 \mathrm{~Hz}, 1 \mathrm{H}), 7.43(\mathrm{t}$, $J=7.6 \mathrm{~Hz}, 1 \mathrm{H}), 7.30(\mathrm{~d}, J=7.5 \mathrm{~Hz}, 1 \mathrm{H}), 7.19-7.08(\mathrm{~m}, 10 \mathrm{H}), 7.07-6.97(\mathrm{~m}, 8 \mathrm{H}), 6.60(\mathrm{~d}, J=7.7 \mathrm{~Hz}, 1 \mathrm{H}), 6.58(\mathrm{~s}, 1 \mathrm{H})$, $2.49(\mathrm{~s}, 3 \mathrm{H}), 2.30(\mathrm{~s}, 6 \mathrm{H}), 2.28(\mathrm{~s}, 3 \mathrm{H}), 2.27(\mathrm{~s}, 3 \mathrm{H}), 2.21$ (s, 3H) ppm. ${ }^{13} \mathrm{C}$ NMR $\left(126 \mathrm{MHz}, \mathrm{CDCl}_{3}\right) \delta 145.8,143.8$, 143.1, 139.6, 139.5, 139.4, 137.9 (2C), 137.7, 136.8, 136.6, 136.5, 132.4, 132.3, 132.0, 131.6 (2C), $131.4,129.3$ (2C), 129.0, 128.7, 128.6, 128.4, 128.3, 128.2 (2C), 128.0, 127.9 (2C), 127.8, 127.5 (3C), 127.2, (2C), 125.8, 124.8, 123.6 (2C), 123.3, 123.2, 98.8, 98.6, 97.5, 89.1, 89.0, 88.4, 21.8, 21.5 (2C), 21.5, 21.3 (3C) ppm. IR (ATR) $\tilde{v}=3037$, 2918, 2860, 2211, 1942, 1601, 1581, 1485, 1450, 1392, 1263, 1168, 1091, 1038, 906, 881, 781, 744, 704, 689, $641,598 \mathrm{~cm}^{-1}$. HRMS $\left(\mathrm{EI}^{+}\right) \mathrm{m} / \mathrm{z}$ for $\mathrm{C}_{48} \mathrm{H}_{30}[\mathrm{M}]^{+}$: calc:690.3286, found:690.3289. Melting point: $66-69{ }^{\circ} \mathrm{C}$. 


\section{3,3'--Dimethoxy-4'-(3-methoxyphenyl)-3',5',6'-tris((3-methoxyphenyl)ethynyl)-1,1':2',1"-terphenyl (14i)}

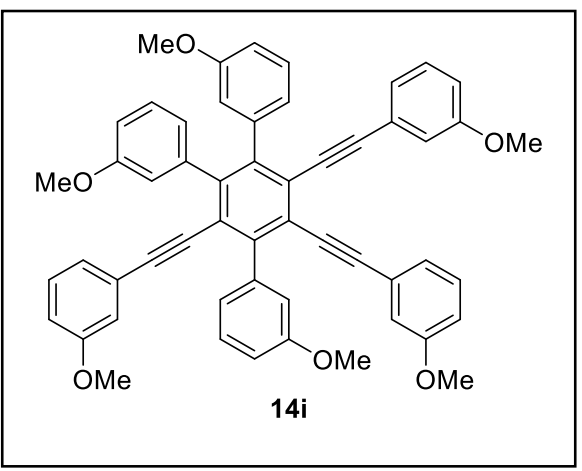

According to General Procedure 4 the title compound was prepared using 1,4-bis(3-methoxyphenyl)buta-1,3-diyne $(787 \mathrm{mg}, 3.00 \mathrm{mmol}$, 1.00 equiv.), $\mathrm{CoBr}_{2}$ (dppe) (92.6 mg, $0.15 \mathrm{mmol}, 0.05$ equiv.), zinc (19.6 mg, $0.30 \mathrm{mmol}, 0.10$ equiv.) and zinc(II)iodide (96.0 mg, 0.30 mmol, 0.10 equiv.) in $3.00 \mathrm{~mL}$ THF. The product was obtained after column chromatography ( $n$-pentane: $\mathrm{CH}_{2} \mathrm{Cl}_{2}=1: 1$ ) as orange-yellow glassy solid (623 mg, $0.79 \mathrm{mmol}, 79 \%)$.

${ }^{1} \mathrm{H}$ NMR $\left(500 \mathrm{MHz}, \mathrm{CDCl}_{3}\right) \delta 7.47(\mathrm{t}, J=7.9 \mathrm{~Hz}, 1 \mathrm{H}), 7.34-7.27(\mathrm{~m}, 2 \mathrm{H})$, 7.23-7.15 (m, 4H), 7.09-7.04 (m, 2H), 6.96-6.90 (m, 2H), 6.89-6.73 (m, 9H), $6.47(\mathrm{dt}, J=7.6,1.2 \mathrm{~Hz}, 1 \mathrm{H}), 6.32(\mathrm{dd}$, $J=2.7,1.3 \mathrm{~Hz}, 1 \mathrm{H}), 3.87(\mathrm{~s}, 3 \mathrm{H}), 3.75(\mathrm{~s}, 2 \mathrm{H}), 3.72(\mathrm{~s}, 2 \mathrm{H}), 3.71(\mathrm{~s}, 2 \mathrm{H}), 3.70(\mathrm{~s}, 2 \mathrm{H}), 3.69(\mathrm{~s}, 3 \mathrm{H}) .{ }^{13} \mathrm{C}$ NMR $(126$ $\left.\mathrm{MHz}_{1} \mathrm{CDCl}_{3}\right) \delta 159.5,159.4,159.2,159.1,159.0,158.9,146.0,143.5,143.0,140.8,140.7,140.6,129.5,129.4$, $129.2,128.6,128.5,128.4,125.7,124.9,124.5(2 \mathrm{C}), 124.3,124.1$ (2C), 123.8, 123.5 (2C), 123.2, 123.1, 116.2, $116.1,115.9$ (2C), 115.8, 115.6, 115.5 (3C), 115.4, 114.3, 113.5, 98.8, 98.7, 97.6, 88.8, 88.7, 88.1, 55.5, 55.4 (2C), 55.3 (2C), 55.2 ppm. IR (ATR) $\tilde{v}=3001,2957,2938,2834,2211,1597,1576,1486,1462,1427,1348,1316,1283$, 1229, 1209, 1158, 1080, 1040, 994, 966, 915, 859, 779, 736, 700, 685, 623, 606, 591, $559 \mathrm{~cm}^{-1}$. HRMS (EI+) m/z for $\mathrm{C}_{54} \mathrm{H}_{42} \mathrm{O}_{6}[\mathrm{M}]^{+}$: calc: 786.2976 , found: 786.2982 .

1,2,4-Tri(pent-1-yn-1-yl)-3,5,6-tri-n-propylbenzene (14j) and 1,3,5-tri(pent-1-yn-1-yl)-2,4,6-tri-n-propylbenzene (15j)
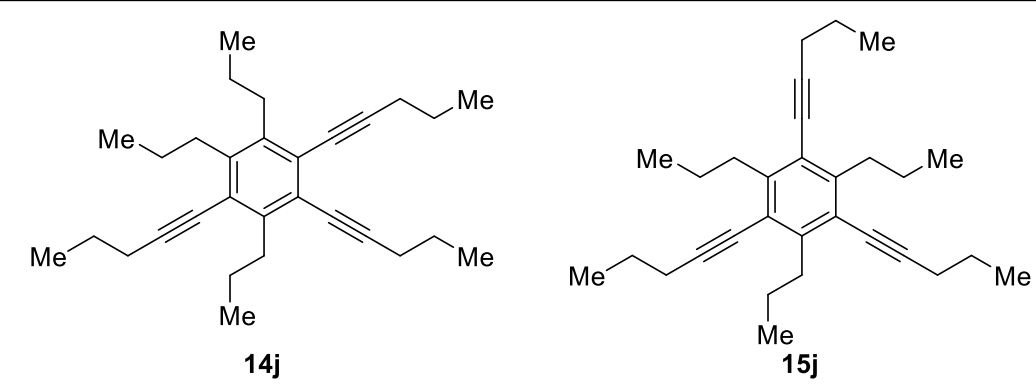

According to General Procedure 4 the title compounds were prepared using deca-4,6-diyne (363 mg, $2.70 \mathrm{mmol}$, 1.00 equiv.), $\mathrm{CoBr}_{2}$ (dppe) (166 mg, $0.27 \mathrm{mmol}, 0.10$ equiv.), zinc ( $35 \mathrm{mg}, 0.54 \mathrm{mmol}, 0.20$ equiv.) and zinc(II)iodide (87.0 mg, 0.54 mmol, 0.20 equiv.) in $3.00 \mathrm{~mL}$ THF. After column chromatography (eluent: $n$-pentane) the product 14j was obtained as a yellow oil (122 $\mathrm{mg}, 0.91 \mathrm{mmol}, 34 \%$ ) and the minor isomer $15 \mathbf{j}$ was obtained as a yellow oil (58 mg, $0.43 \mathrm{mmol}, 16 \%)$.

Analytical data of the major isomer $(\mathbf{1 4} \mathbf{j})$ :

${ }^{1} \mathrm{H}$ NMR $\left(300 \mathrm{MHz} \mathrm{CDCl}_{3}\right) \delta$ 2.97-2.86 (m, 2H), 2.82-2.68 (m, 4H), 2.53-2.40 (m, 6H), 1.72-1.48 (m, $\left.12 \mathrm{H}\right), 1.12-$ 1.04 (m, 9H), 1.05 - 0.91 (m, 9H) ppm. $\left.{ }^{13} \mathrm{C} \mathrm{NMR} \mathrm{(75} \mathrm{MHz,} \mathrm{CDCl}\right)$ ) $144.5,142.1,140.2,125.5,123.8,123.1,97.6$, 97.5, 96.2, 79.7, 79.1, 78.7, 36.0, 34.2, 34.0, 24.0, 23.9, 23.1, 22.6, 22.5, 22.5, 22.1, 22.0, 21.9, 14.8, 14.8, 14.6, 13.8, 13.8, 13.8 ppm. IR (ATR) $\tilde{v}=2960,2931,2870,1461,1423,1378,1337,1273,1090,886,748,608,587 \mathrm{~cm}^{-1}$. HRMS $\left(\mathrm{EI}^{+}\right) \mathrm{m} / \mathrm{z}$ for $\mathrm{C}_{30} \mathrm{H}_{42}[\mathrm{M}]^{+}$: calc: 402.3281 , found: 402.3269 . 
Analytical data of the minor isomer (15j):

${ }^{1} \mathrm{H}$ NMR $\left(300 \mathrm{MHz}, \mathrm{CDCl}_{3}\right) \delta 2.99-2.89(\mathrm{~m}, 6 \mathrm{H}), 2.44(\mathrm{t}, J=6.9 \mathrm{~Hz}, 6 \mathrm{H}), 1.72-1.56(\mathrm{~m}, 12 \mathrm{H}), 1.07(\mathrm{t}, J=7.4 \mathrm{~Hz}, 9 \mathrm{H})$, $0.97(\mathrm{t}, J=7.3 \mathrm{~Hz}, 9 \mathrm{H}) \mathrm{ppm} .{ }^{13} \mathrm{C}$ NMR $\left(75 \mathrm{MHz}, \mathrm{CDCl}_{3}\right) \delta 146.1,121.1,96.1,78.1,36.2,23.3,22.6,21.8,14.7,13.8$ ppm. IR (ATR) $\tilde{v}=2959,2930,2869,1708,1459,1417,1379,1336,1275,1228,1157,1085,1030,975,886,748$ $\mathrm{cm}^{-1}$. HRMS $\left(\mathrm{El}^{+}\right) \mathrm{m} / \mathrm{z}$ for $\mathrm{C}_{30} \mathrm{H}_{42}[\mathrm{M}]^{+}$: calc: 402.3281 , found: 402.3269 .

\section{(3,5,6-Tris((trimethylsilyl)ethynyl)benzene-1,2,4-triyl)tris(trimethylsilane) (14k)}

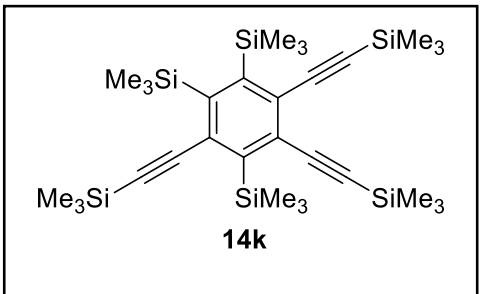

According to General Procedure 4 the title compound was prepared using 1,4-bis(trimethylsilyl)butadiyne (583 mg, $3.00 \mathrm{mmol}, 1.00$ equiv.), $\mathrm{CoBr}_{2}$ (dppe) (92.6 mg, $0.15 \mathrm{mmol}, 0.05$ equiv.), zinc (19.6 mg, $0.30 \mathrm{mmol}$, 0.10 equiv.) and zinc(II)iodide ( $96.0 \mathrm{mg}, 0.30 \mathrm{mmol}, 0.10$ equiv.) in $3.00 \mathrm{~mL}$ THF. The product was obtained after column chromatography (eluent: $n$-pentane) as a light-orange solid (174 $\mathrm{mg}, 0.30 \mathrm{mmol}, 30 \%)$.

${ }^{1} \mathrm{H}$ NMR $\left(500 \mathrm{MHz}, \mathrm{CDCl}_{3}\right) \delta 0.50(\mathrm{~s}, 9 \mathrm{H}), 0.45(\mathrm{~s}, 9 \mathrm{H}), 0.41(\mathrm{~s}, 9 \mathrm{H}), 0.28(\mathrm{~s}, 10 \mathrm{H}), 0.27(\mathrm{~s}, 9 \mathrm{H}), 0.25(\mathrm{~s}, 9 \mathrm{H}) \mathrm{ppm}$. ${ }^{13} \mathrm{C}$ NMR $\left(126 \mathrm{MHz}, \mathrm{CDCl}_{3}\right) \delta 153.9,153.4,143.0,134.2,131.6,130.6,108.6,105.9,105.4,105.3,105.2,103.8$, 3.2, 2.7, 2.6, 0.1 (2C), -0.4 ppm. IR (ATR) $\tilde{v}=2959,2899,2141,1454,1408,1307,1246,1184,1018,918,829$, 757, 697, 681, 647, 635, 603, 589, $567 \mathrm{~cm}^{-1}$. HRMS (El+) m/z for $\mathrm{C}_{30} \mathrm{H}_{54} \mathrm{Si}_{6}\left[\mathrm{M}^{+}\right.$: calc: 582.2836, found: 582.2841 . Melting point: $125-128^{\circ} \mathrm{C}$.

\section{2,2"-Dimethyl-4'-(o-tolyl)-3',5',6'-tris(o-tolylethynyl)-1,1':2',1"-terphenyl (14I)}

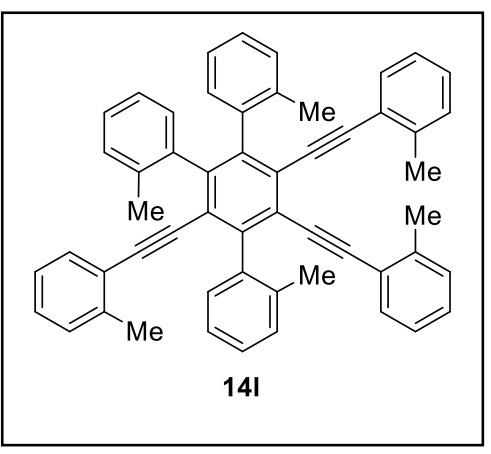

According to General Procedure 4 the title compound was prepared using 1,4-di-m-tolylbutadiyne (691 mg, $3.00 \mathrm{mmol}, 1.00$ equiv), [CoBr 2 (dppe)] (92.6 mg, $0.15 \mathrm{mmol}, 0.05$ equiv), zinc ( $19.6 \mathrm{mg}, 0.30 \mathrm{mmol}, 0.10$ equiv) and zinc(II)iodide ( $96.0 \mathrm{mg}, 0.30 \mathrm{mmol}, 0.10$ equiv) in $3.00 \mathrm{~mL}$ THF. The product was obtained after filtration over a short plug of silica (eluent: $n$-pentane: $\mathrm{CH}_{2} \mathrm{Cl}_{2}$ 3:1) as a light brown solid (666 mg, $0.97 \mathrm{mmol}, 97 \%$ ).

${ }^{1} \mathbf{H}$ NMR $\left(500 \mathrm{MHz}, \mathrm{CDCl}_{3}\right) \delta 7.46-7.39(\mathrm{~m}, 2 \mathrm{H}), 7.36-7.30(\mathrm{~m}, 5 \mathrm{H}), 7.31-$ $7.18(\mathrm{~m}, 3 \mathrm{H}), 7.17-6.88(\mathrm{~m}, 20 \mathrm{H}), 6.48-6.42(\mathrm{~m}, 1 \mathrm{H}), 2.37-2.34(\mathrm{~m}, 5 \mathrm{H})$, $2.33-2.27(\mathrm{~m}, 3 \mathrm{H}), 2.23(\mathrm{~d}, J=11.4 \mathrm{~Hz}, 4 \mathrm{H}), 1.97(\mathrm{~d}, J=3.2 \mathrm{~Hz}, 3 \mathrm{H}), 1.94(\mathrm{~d}, J=1.8 \mathrm{~Hz}, 3 \mathrm{H}), 1.59(\mathrm{~s}, 3 \mathrm{H}) \mathrm{ppm} .{ }^{13} \mathrm{C}$ NMR $\left(126 \mathrm{MHz}_{2} \mathrm{CDCl}_{3}\right) \delta 146.1,146.0,145.8,143.2,143.1,140.7,140.6,140.5,140.3,140.1$, 139.6, 139.5, 138.7, 136.5, 136.3, 136.3, 132.3, 132.2, 131.6, 131.3, 130.0, 129.8, 129.7, 129.5, 129.4, 129.4, 129.1, 128.4, 128.2, $127.9,127.8,127.5,127.4,127.3,126.0,125.9,125.8,125.4,125.3,125.0,124.9,124.8,124.6,124.3,123.4$, 123.3, 123.1, 97.2, 97.1, 96.4, 96.1, 95.5, 92.4, 92.2, 91.8, 91.6, 91.0, 20.4, 20.3, 20.2, 20.0, 19.9, 19.9, 19.8, 19.8, 19.7, 19.7.ppm. IR (ATR) $\tilde{v}=3059,3022,2921,2207,1599,1486,1454,1401,1379,1264,1197,1159,1113$, 1040, 943, 864, 805, 752, 643, 604, $570 \mathrm{~cm}^{-1}$. HRMS $\left(\mathrm{EI}^{+}\right) \mathrm{m} / z$ for $\mathrm{C}_{48} \mathrm{H}_{30}[\mathrm{M}]^{+}$: calc: 690.3286 , found: 690.3281 . Melting point: $98-102{ }^{\circ} \mathrm{C}$.

*The number and the integration of signals in the NMR spectra mismatches the number of expected signals, possibly due to the hindered rotation of the methyl groups. ${ }^{12}$ 


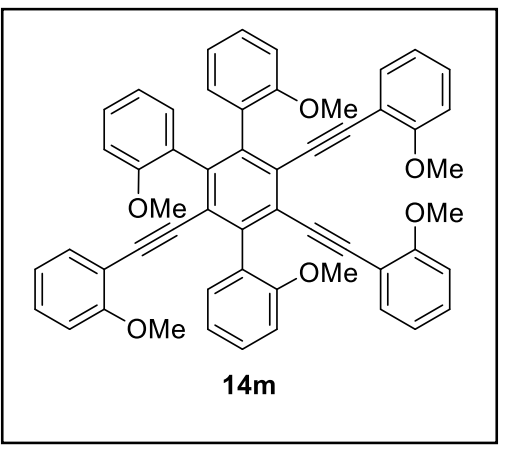

According to General Procedure 4 the title compound was prepared using 1,4-bis(2-methoxyphenyl)buta-1,3-diyne ( $787 \mathrm{mg}, 3.00 \mathrm{mmol}, 1.00$ equiv), $\mathrm{CoBr}_{2}$ (dppe) (92.6 mg, $0.15 \mathrm{mmol}, 0.05$ equiv), zinc (19.6 mg, $0.30 \mathrm{mmol}$, 0.10 equiv) and zinc(II)iodide ( $96.0 \mathrm{mg}, 0.30 \mathrm{mmol}, 0.10$ equiv) in $3.00 \mathrm{~mL}$ THF. The product was obtained after filtration over a short plug of silica (eluent: $n$-pentane: $\mathrm{CH}_{2} \mathrm{Cl}_{2}$ 1:1) as an orange-yellow solid (677 mg, 0.86 $\mathrm{mmol}, 86 \%)$.

${ }^{1} \mathrm{H}$ NMR $(500 \mathrm{MHz} \text {, DMSO-d })_{6} \delta 7.51-7.43(\mathrm{~m}, 1 \mathrm{H}), 7.37$ (ddd, $J=11.4,7.4$, $1.7 \mathrm{~Hz}, 1 \mathrm{H}), 7.33-7.25(\mathrm{~m}, 2 \mathrm{H}), 7.22-7.15(\mathrm{~m}, 4 \mathrm{H}), 7.13-6.92(\mathrm{~m}, 7 \mathrm{H}), 6.91-6.76(\mathrm{~m}, 6 \mathrm{H}), 6.72(\mathrm{t}, J=7.5 \mathrm{~Hz}$, $1 \mathrm{H}), 6.46-6.40(\mathrm{~m}, 1 \mathrm{H}), 3.78-3.75(\mathrm{~m}, 3 \mathrm{H}), 3.69-3.62(\mathrm{~m}, 5 \mathrm{H}), 3.62-3.60(\mathrm{~m}, 3 \mathrm{H}), 3.58-3.55(\mathrm{~m}, 3 \mathrm{H}), 3.52-$ 3.50 (m, 3H) ppm. ${ }^{13}$ C NMR (126 MHz, DMSO-d $\left.d_{6}, 373 \mathrm{~K}\right) \delta$ 159.2, 159.1, 158.9, 156.9, 156.7, 156.2, 156.2, 156.1, $156.1,141.9,141.9,140.5,140.4,140.0,139.9,132.7,132.2,130.8,130.7,129.8,129.6,129.4,129.1,128.5$, 128.4, 128.4, 128.3, 128.2, 128.1, 128.0, 128.0, 128.0, 119.8, 119.7, 119.6, 119.5, 119.5, 118.9, 118.9, 118.8, 112.0, 112.0, 111.9, 111.6, 111.6, 111.3, 110.7, 110.4, 110.3, 110.3, 93.5, 93.4, 92.5, 92.3, 91.7, 91.2, 91.2, 55.2, 55.2, 55.1, 54.9, 54.9, 54.8, 54.7 ppm.* IR (ATR) $\tilde{v}=2934,2836,2209,1597,1581,1494,1462,1435,1407,1278$, $1249,1182,1164,1116,1048,1025,938,805,752 \mathrm{~cm}^{-1}$. HRMS $\left(\mathrm{El}^{+}\right) \mathrm{m} / z$ for $\mathrm{C}_{54} \mathrm{H}_{42} \mathrm{O}_{6}\left[\mathrm{M}^{+}\right.$: calc: 786.2976, found: 786.2981. Melting point: $126-129^{\circ} \mathrm{C}$.

*The number of signals in the NMR spectra mismatches the number of expected signals, possibly due to the hindered rotation of the methoxy groups. ${ }^{12}$ 
NMR spectra of literature unknown products
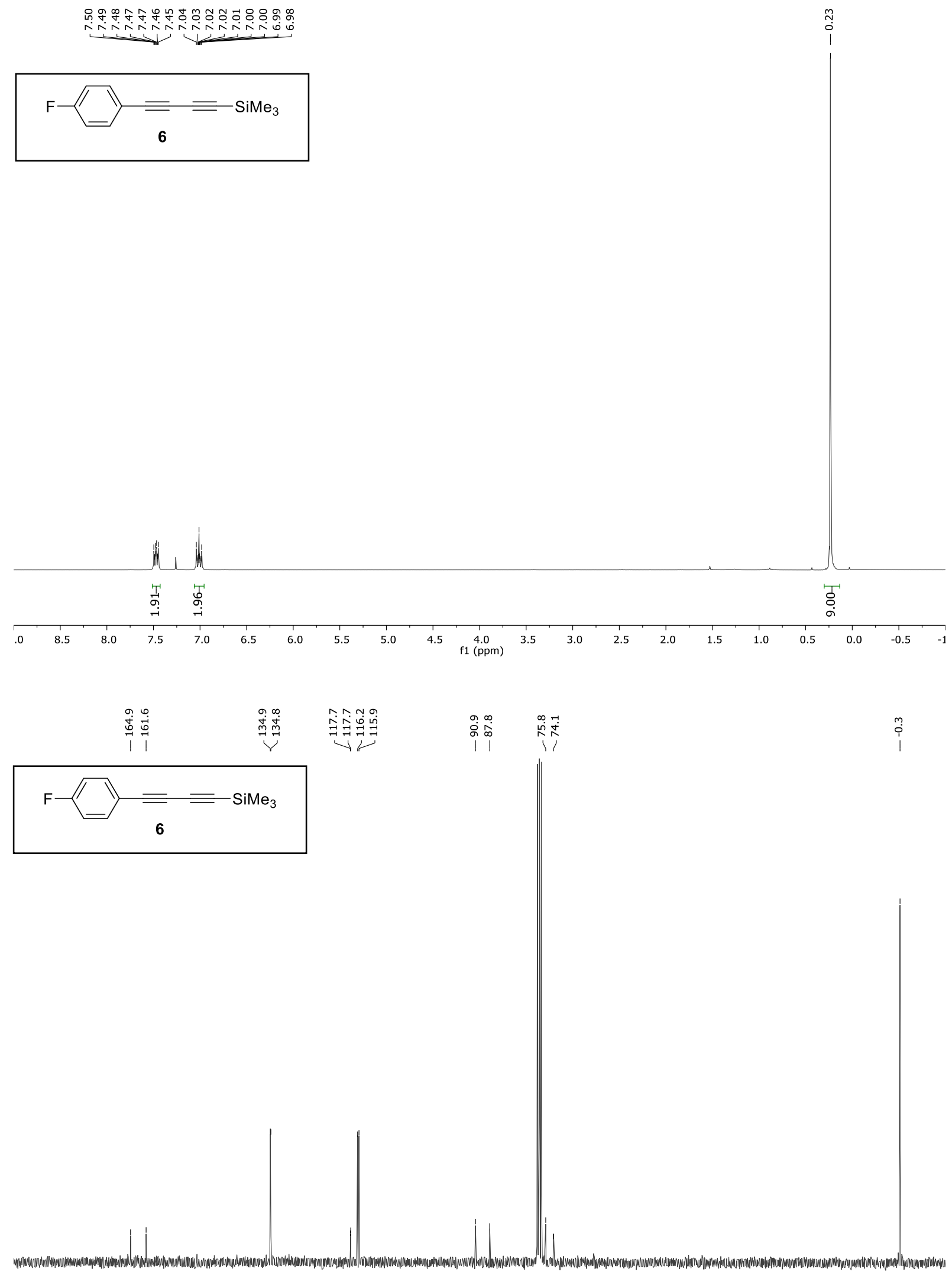

\begin{tabular}{llllllllllllllllllllll}
\hline 90 & 180 & 170 & 160 & 150 & 140 & 130 & 120 & 110 & 100 & 90 & 80 & 70 & 60 & 50 & 40 & 30 & 20 & 10 & 0 & -
\end{tabular} 

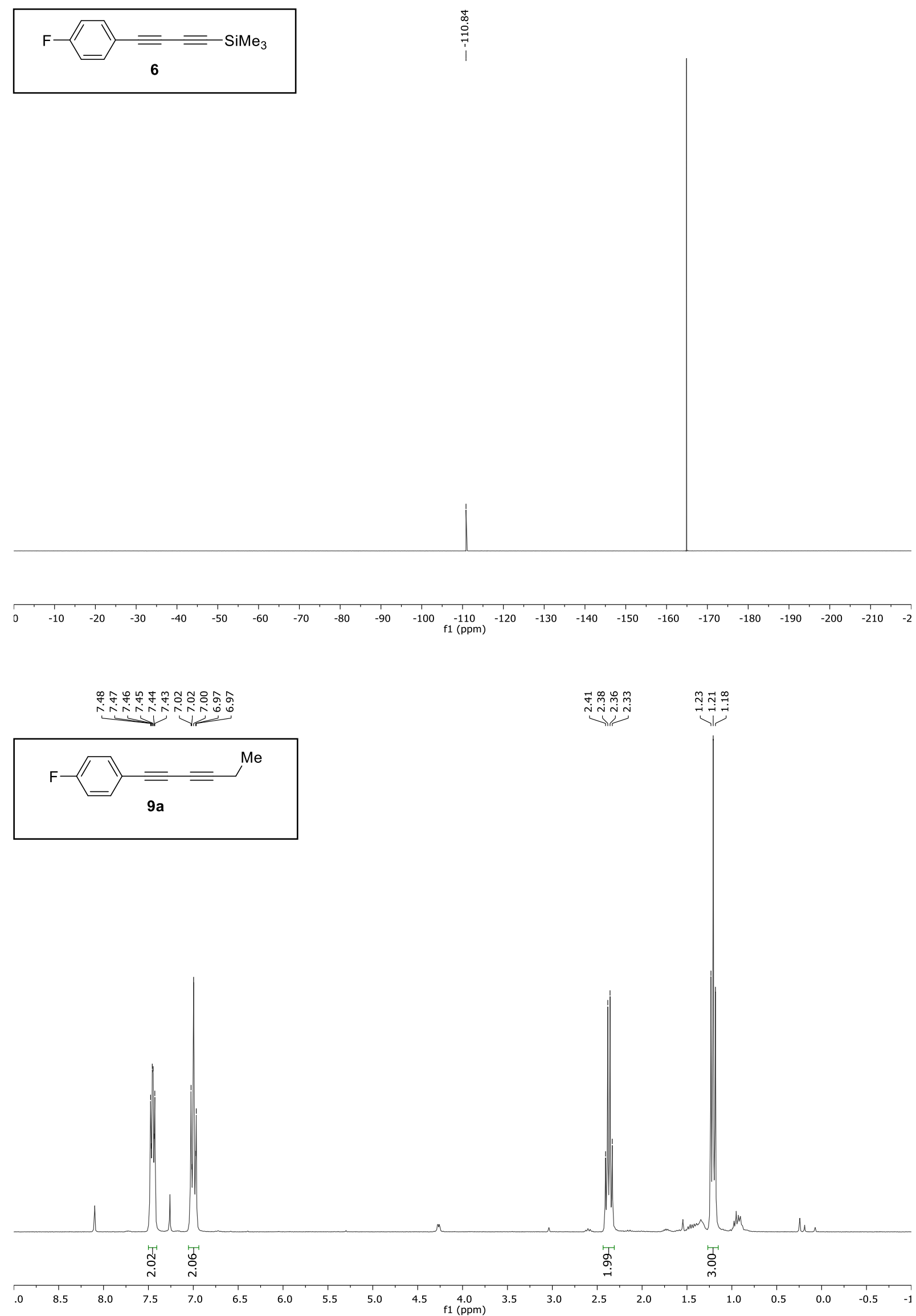


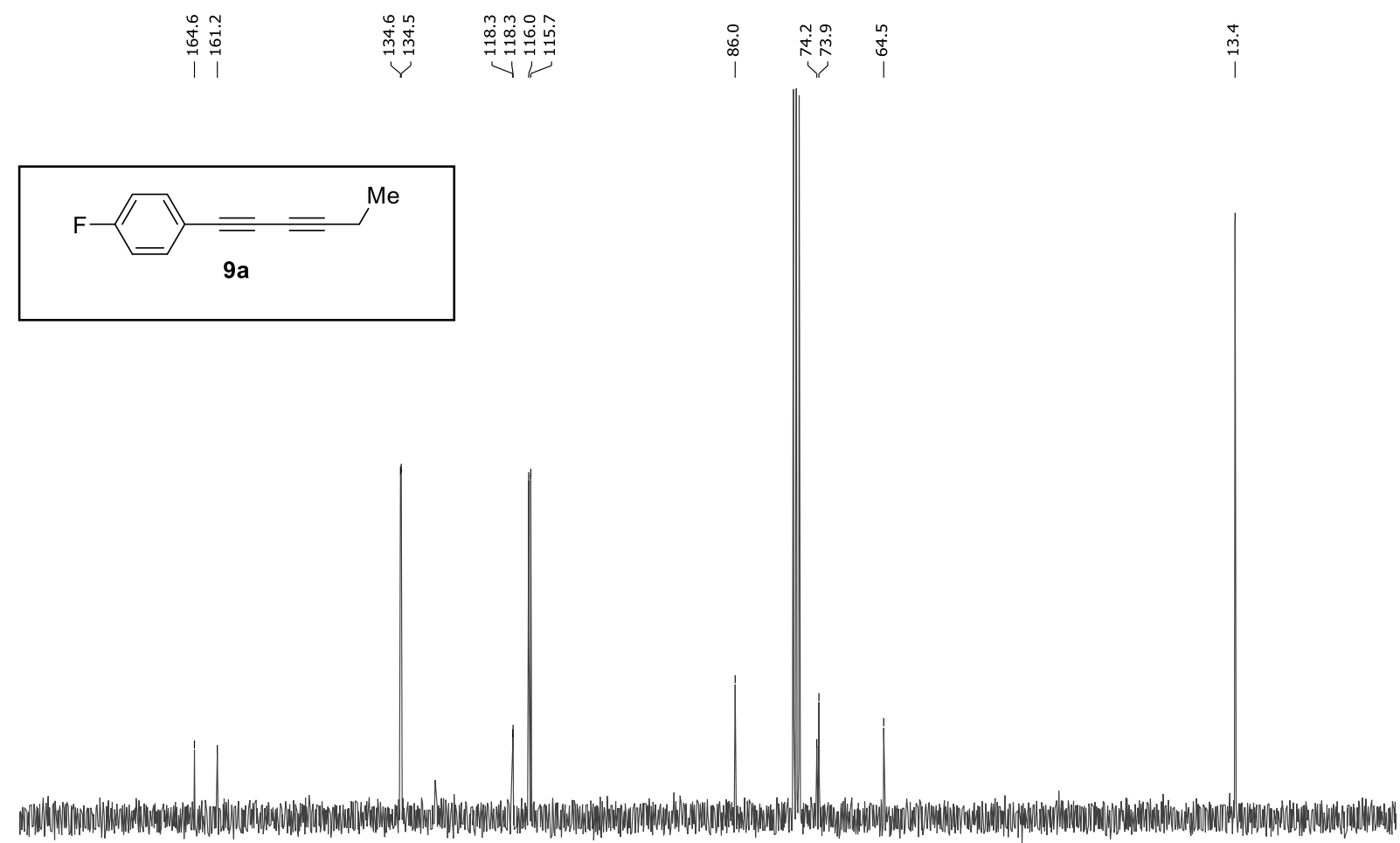

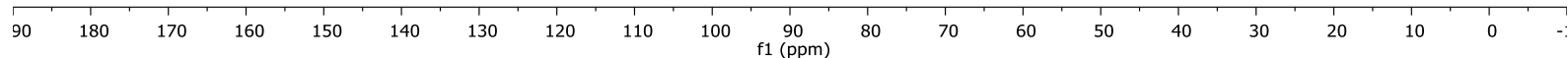

$$
\mathrm{F}=\frac{{ }_{9 a}^{M e}}{=}
$$

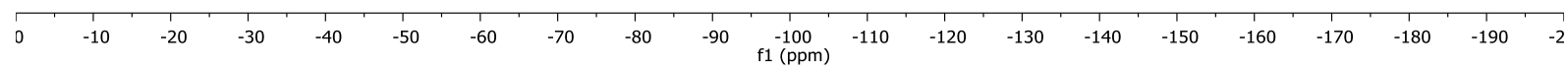



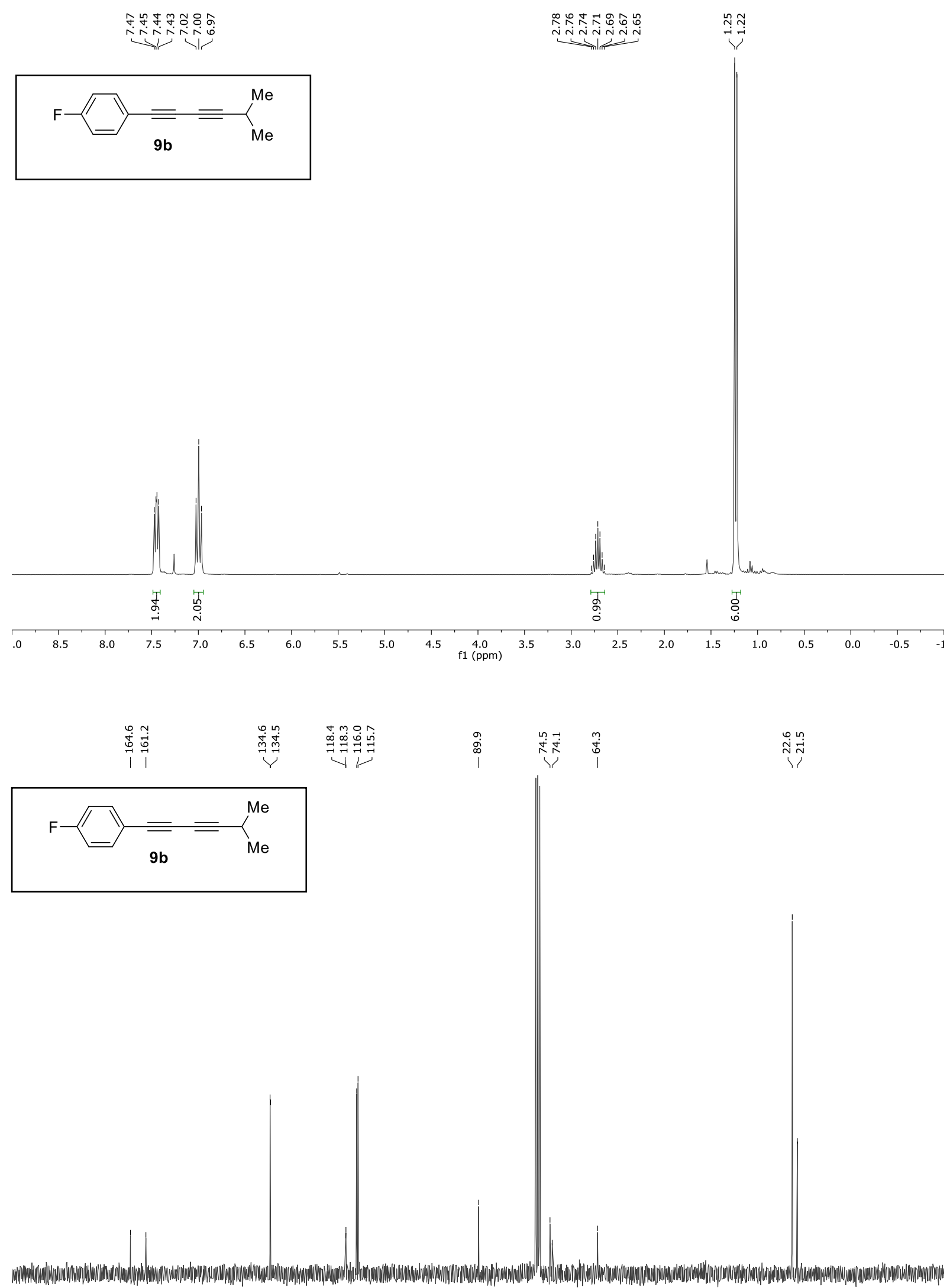

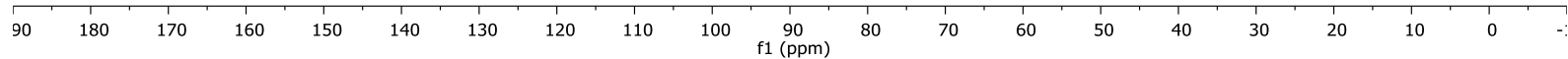




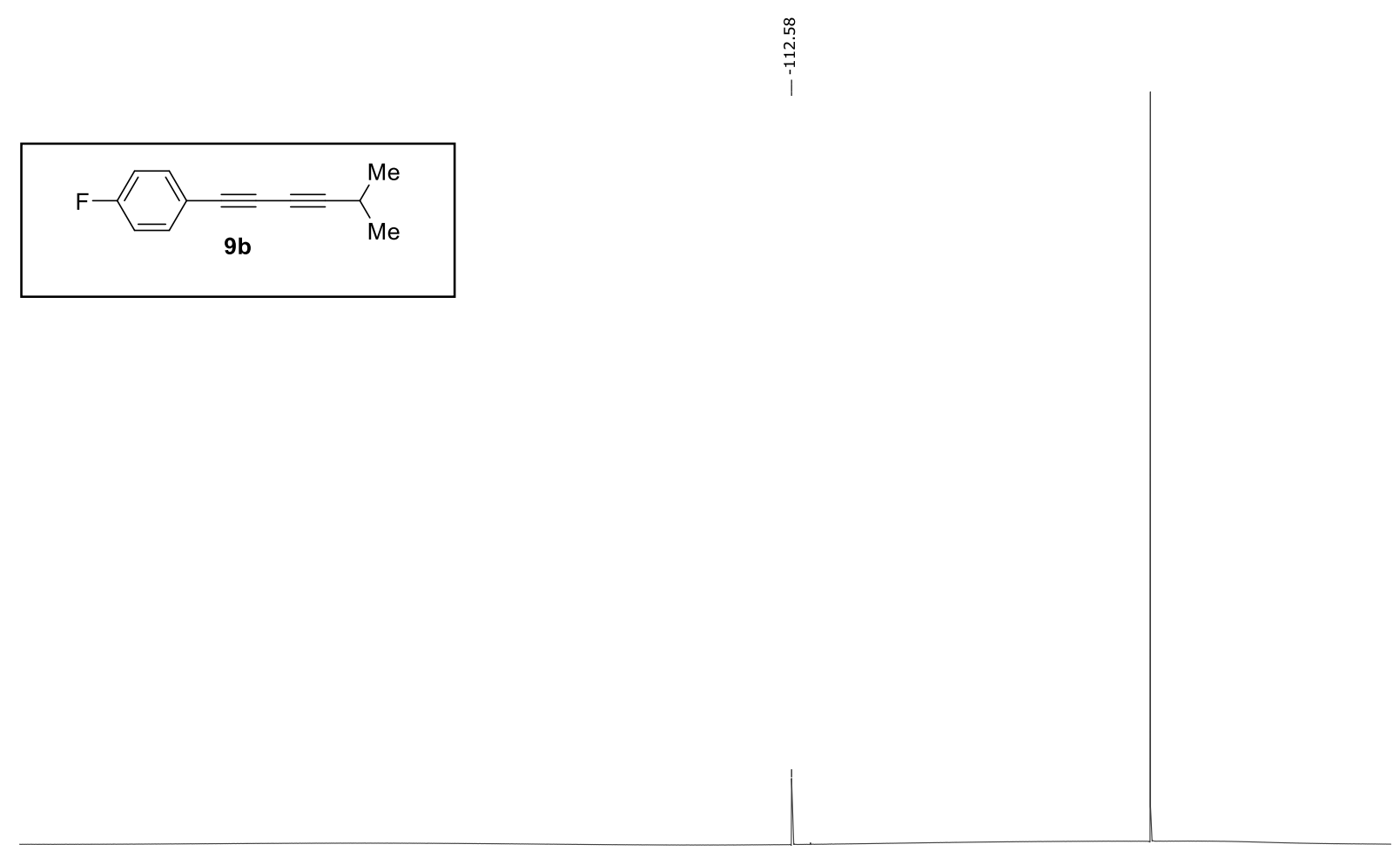

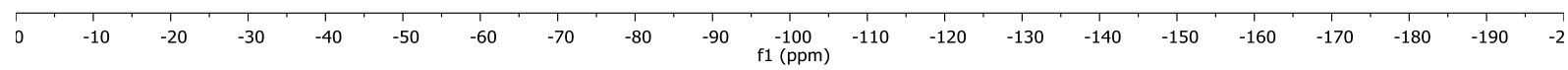

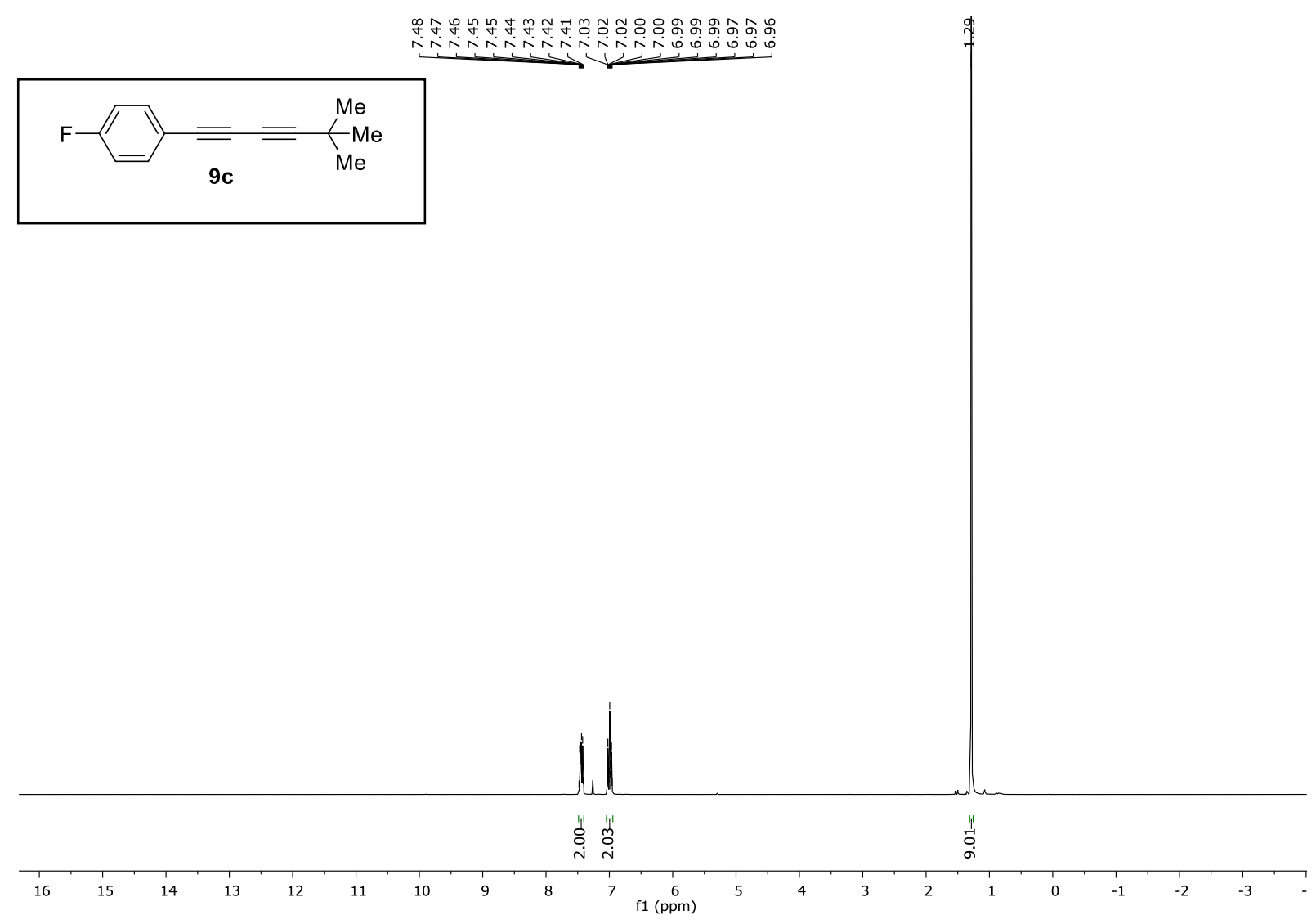




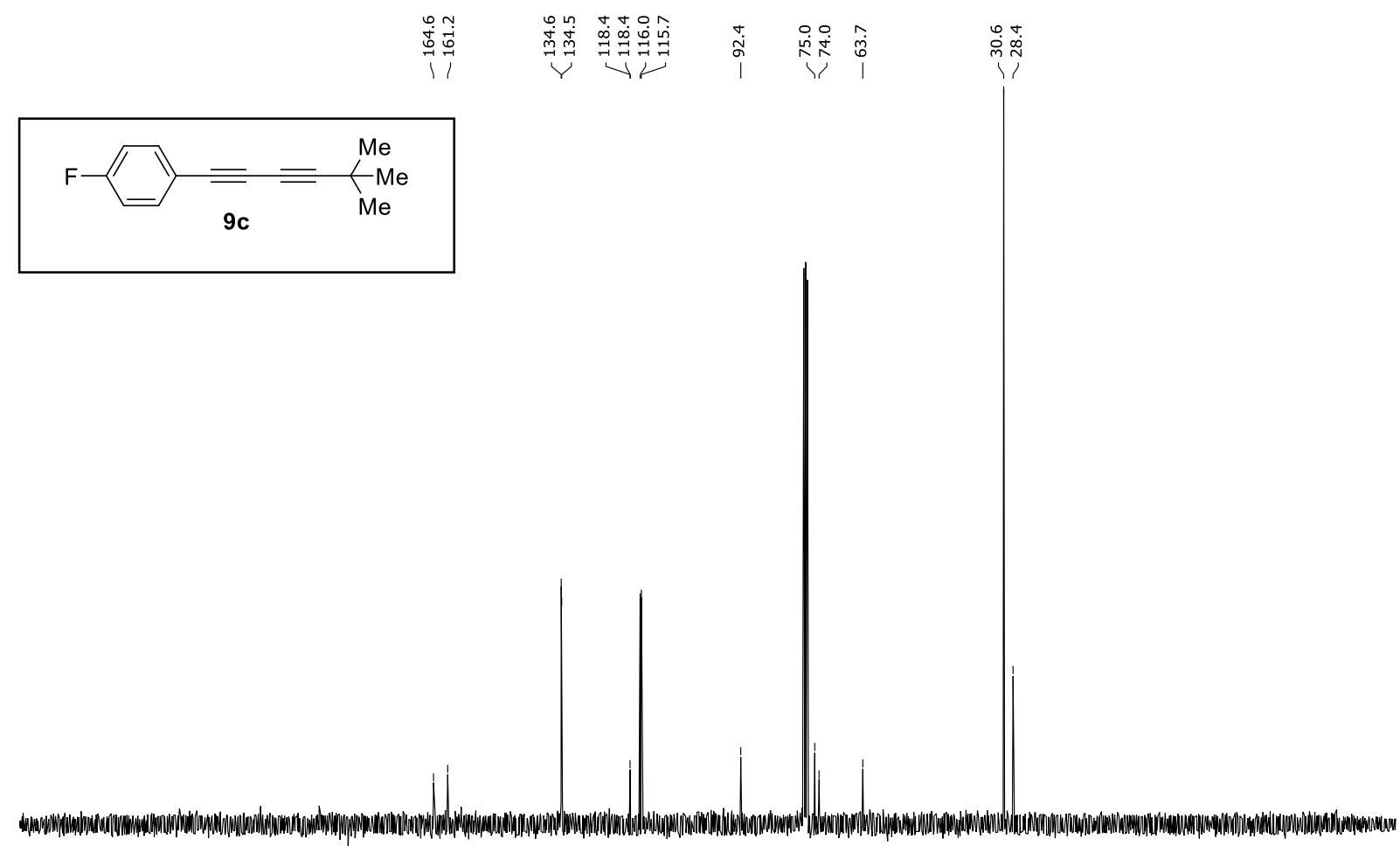

$\begin{array}{lllllllllllllllllllllllllllllllllllllllllll}260 & 250 & 240 & 230 & 220 & 210 & 200 & 190 & 180 & 170 & 160 & 150 & 140 & 130 & 120 & 110 & 100 & 90 & 80 & 70 & 60 & 50 & 40 & 30 & 20 & 10 & 0 & -10 & -20 & -30 & -40 & -50 & -6\end{array}$

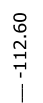

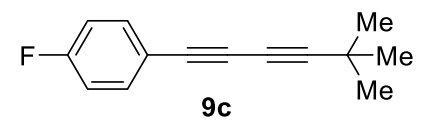

9c

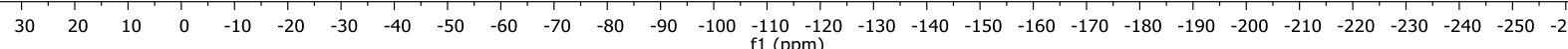




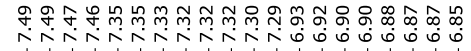

$\stackrel{\substack{\infty \\ i}}{i}$
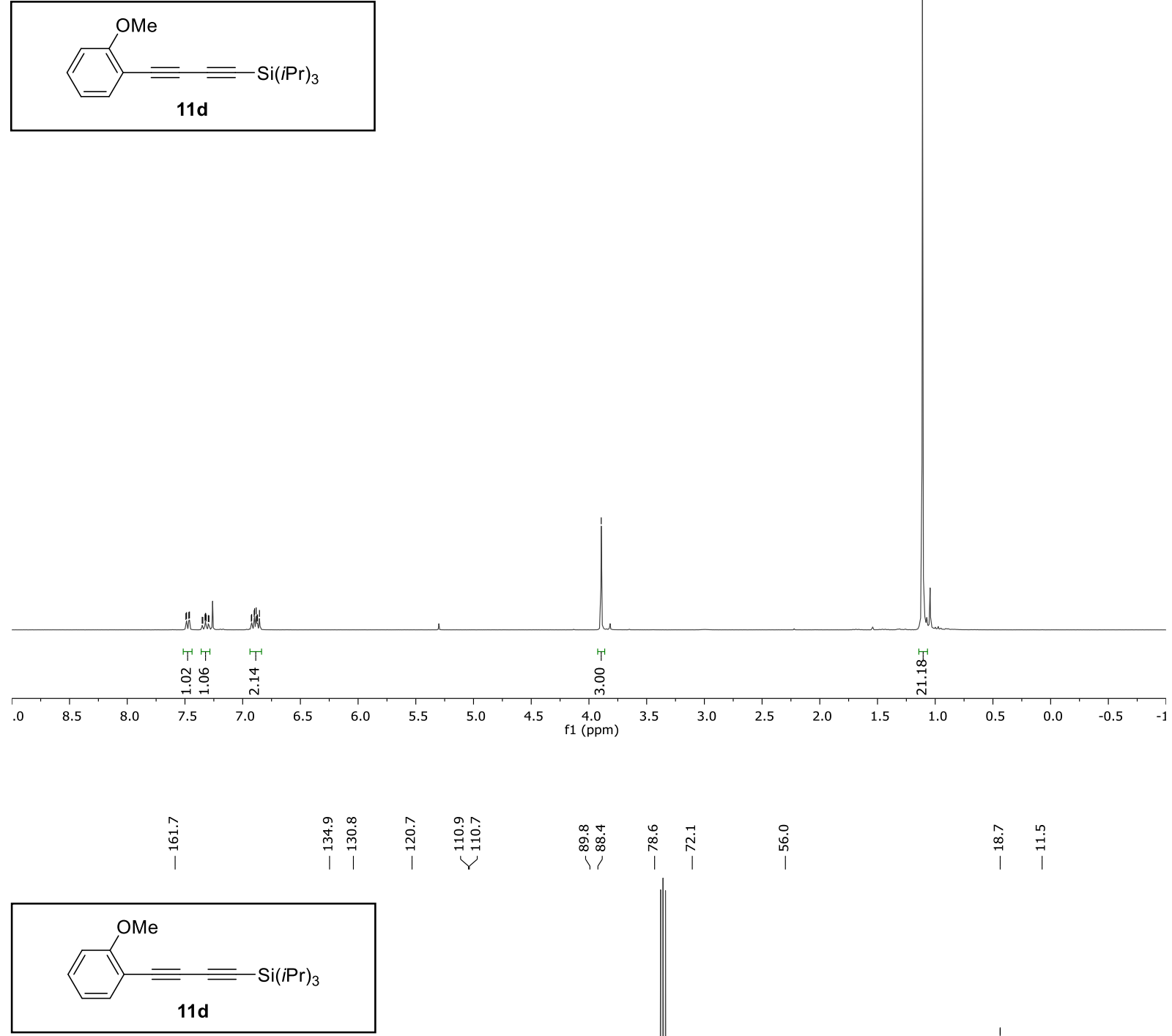

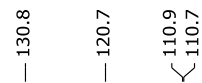

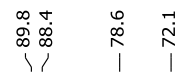

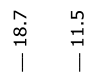

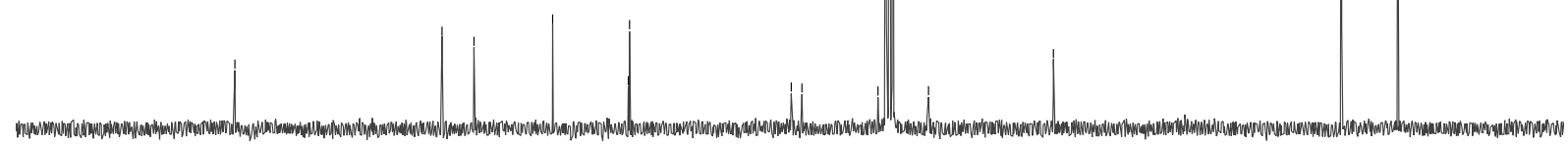



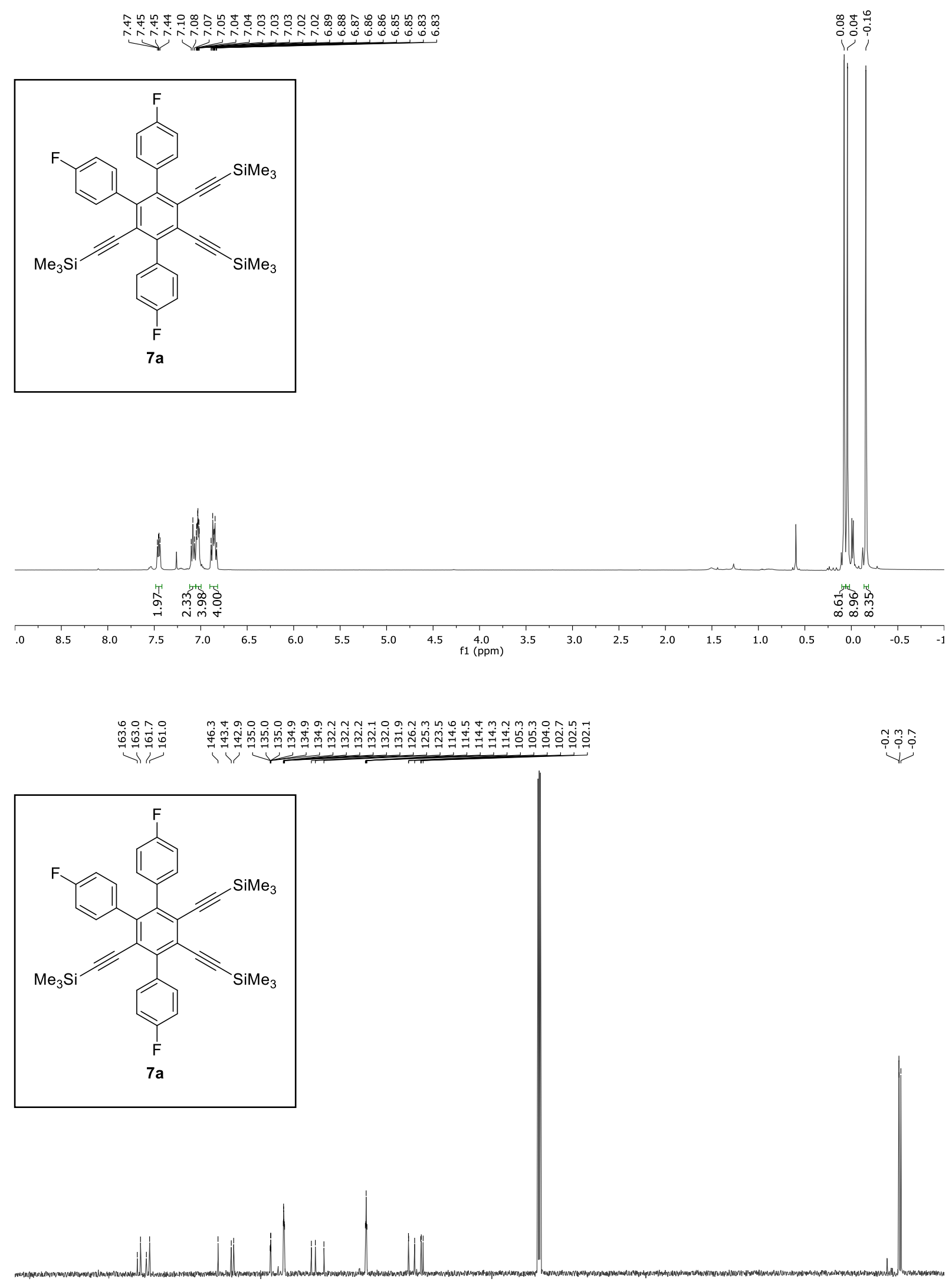

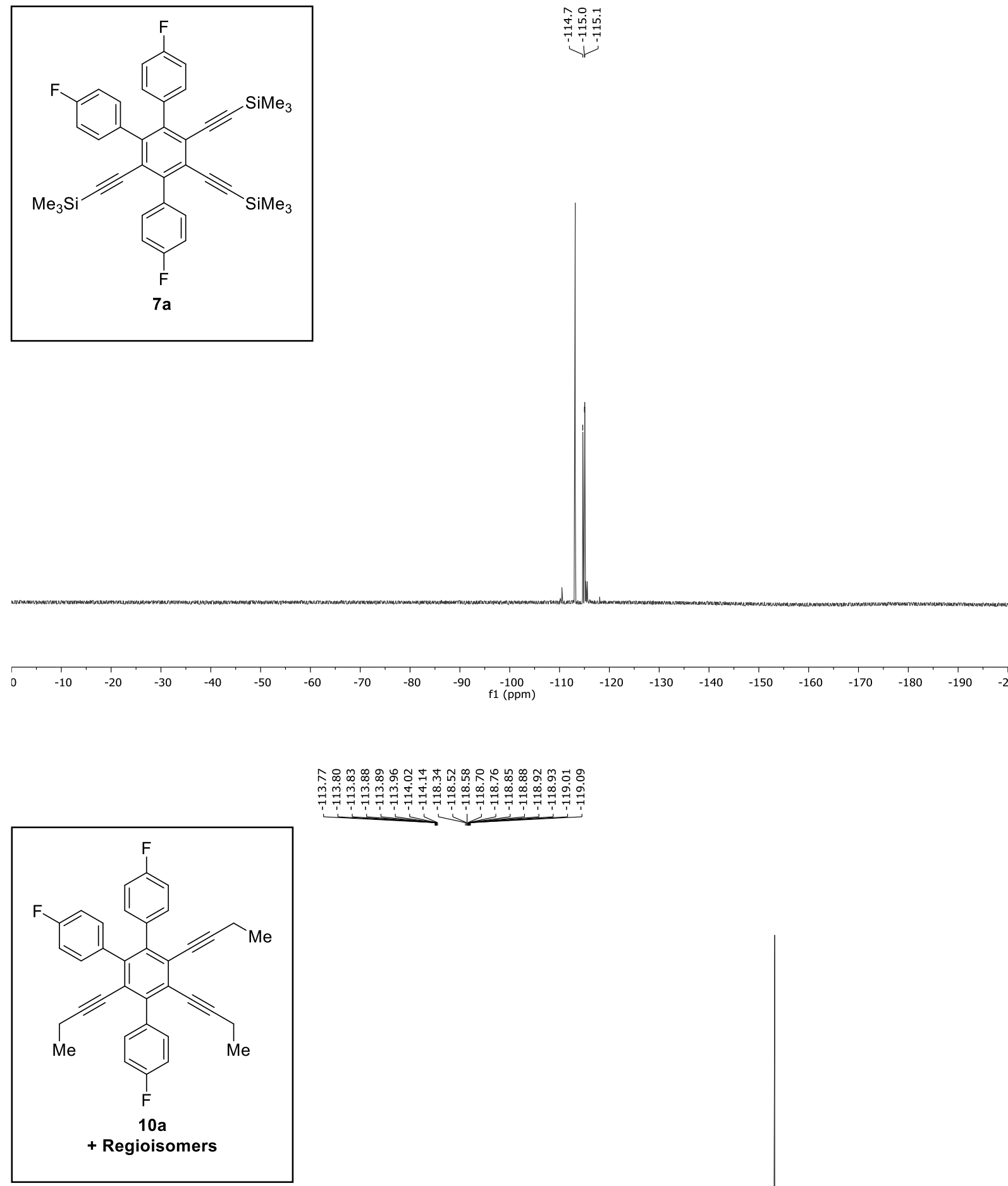

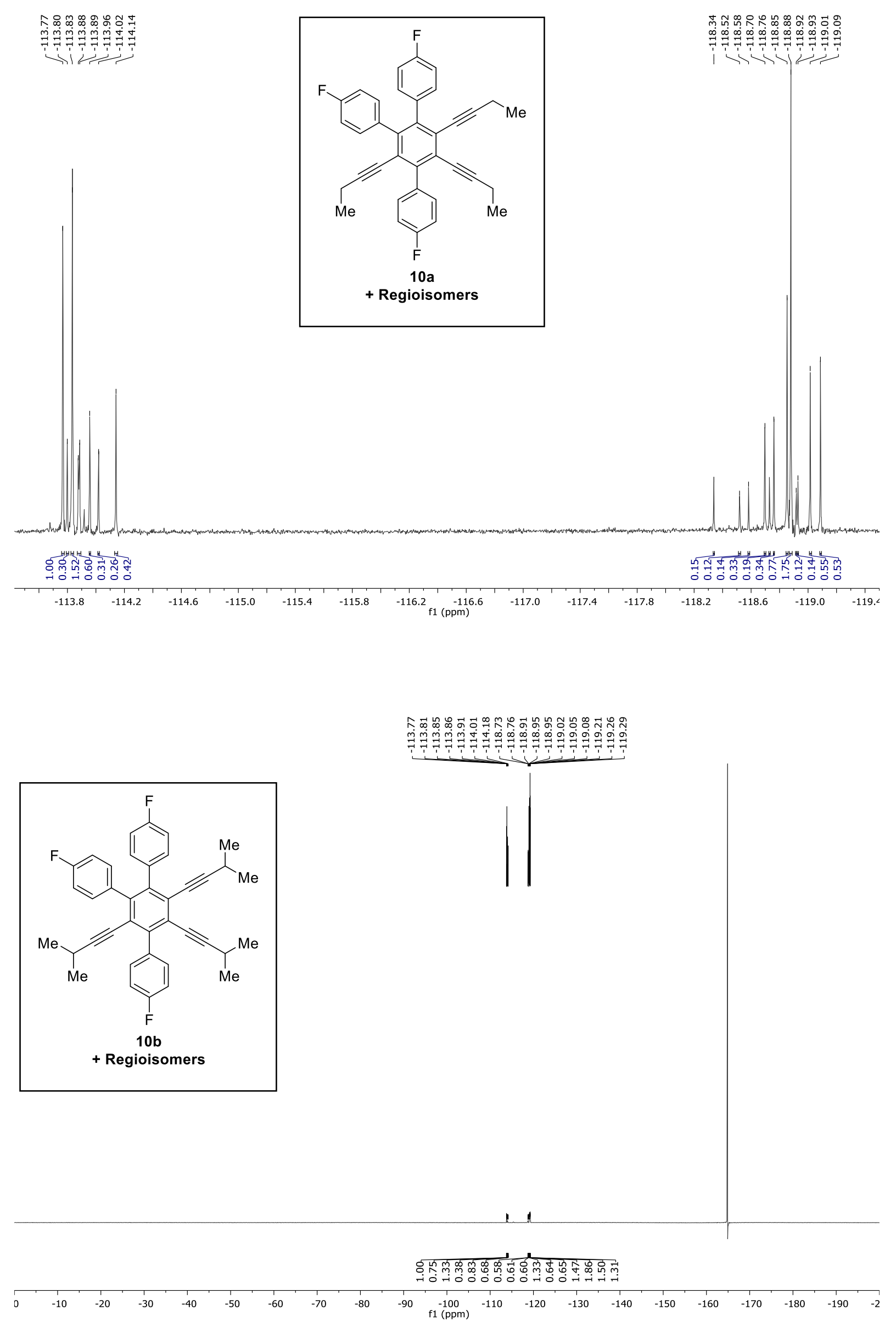

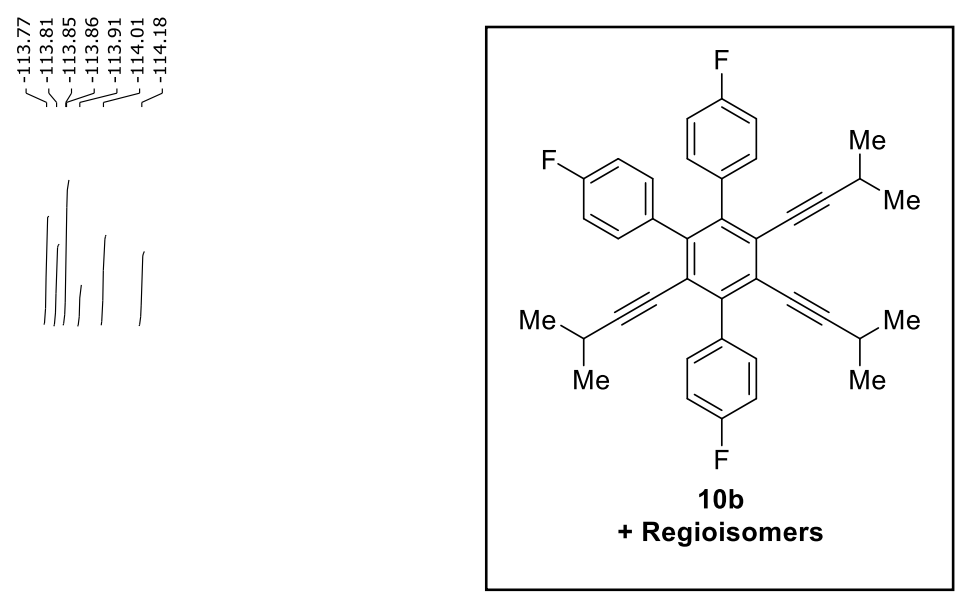

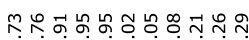

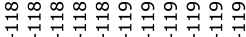
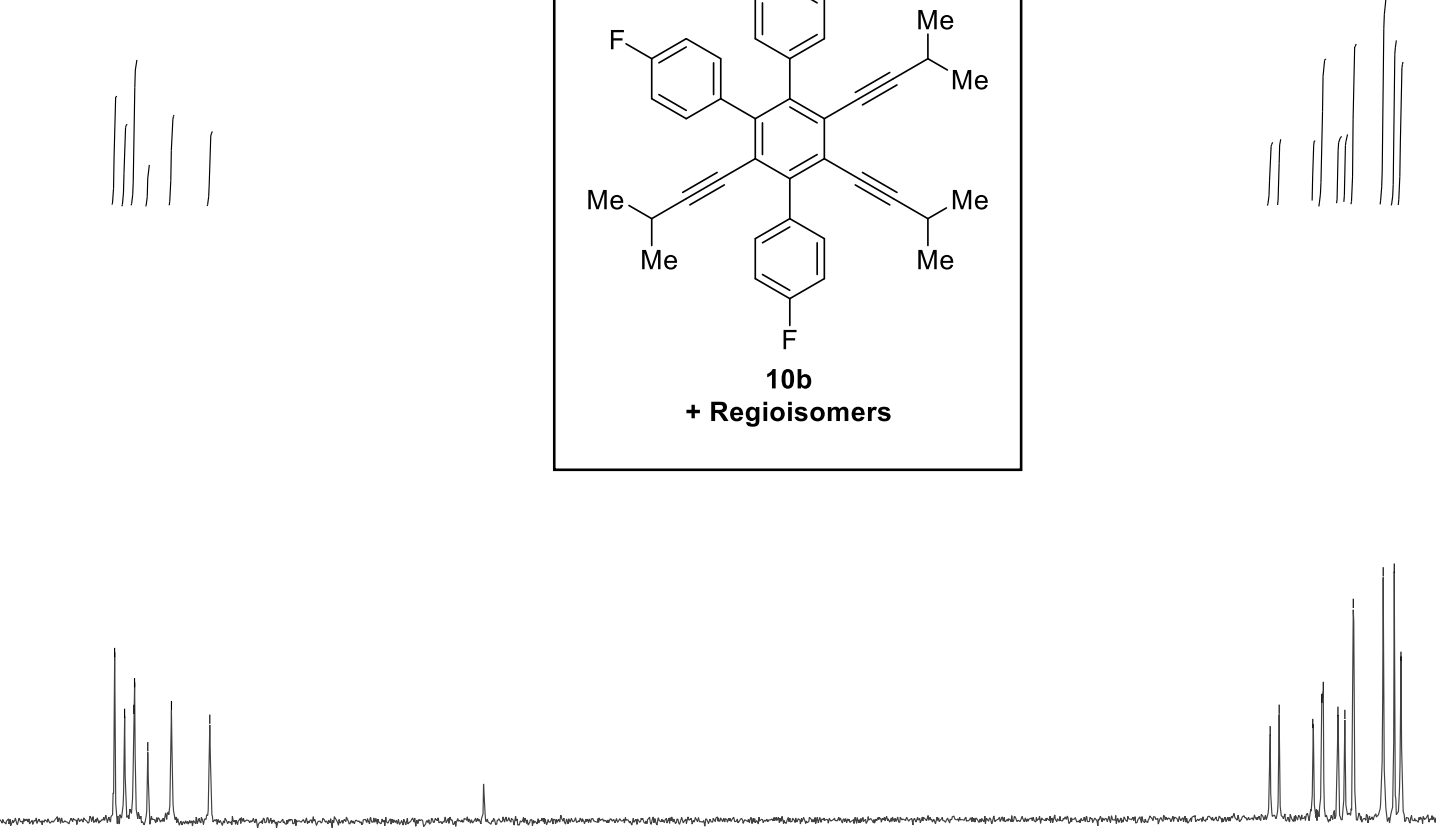

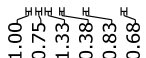

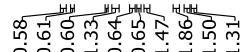

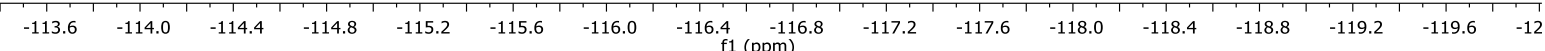
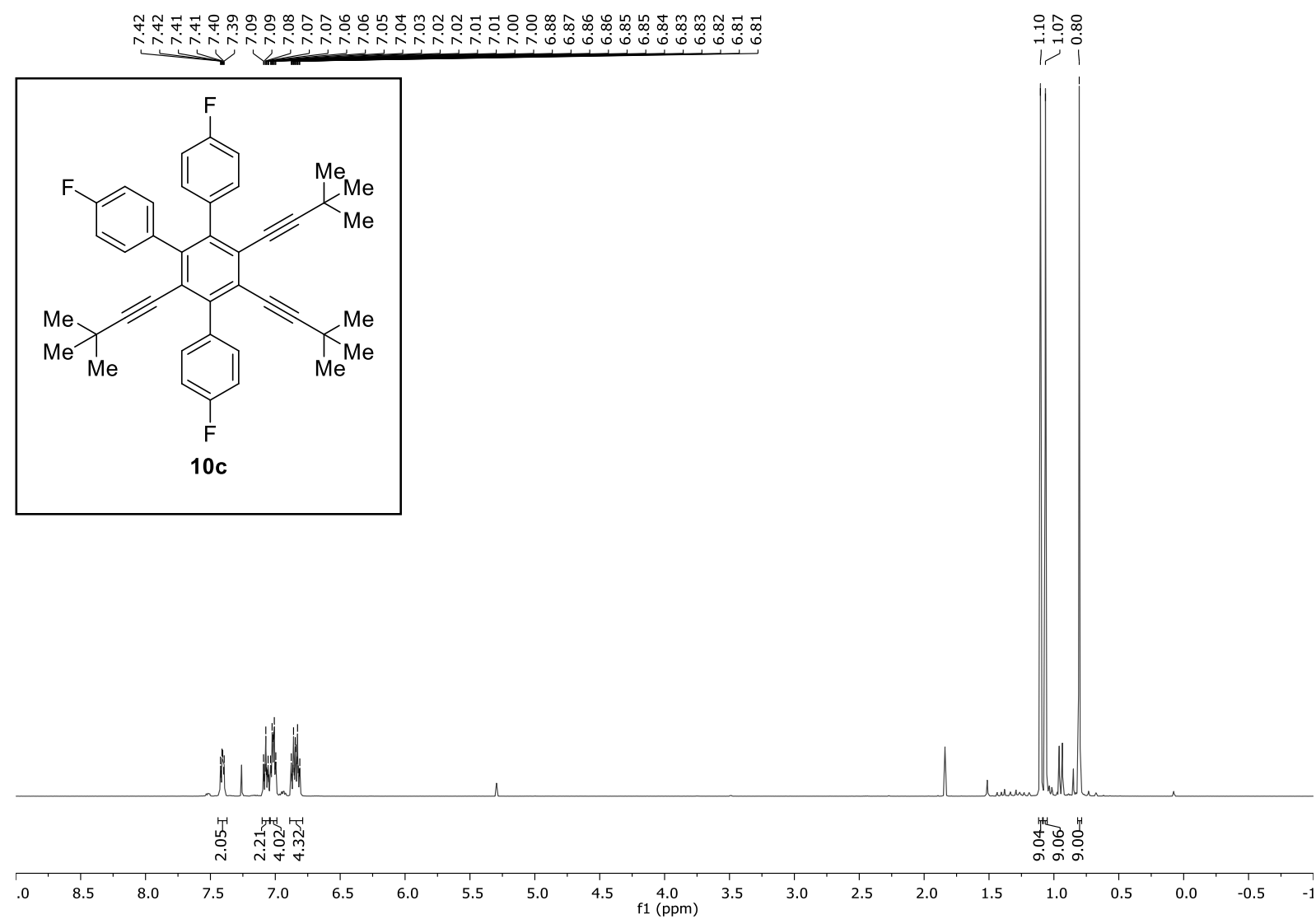

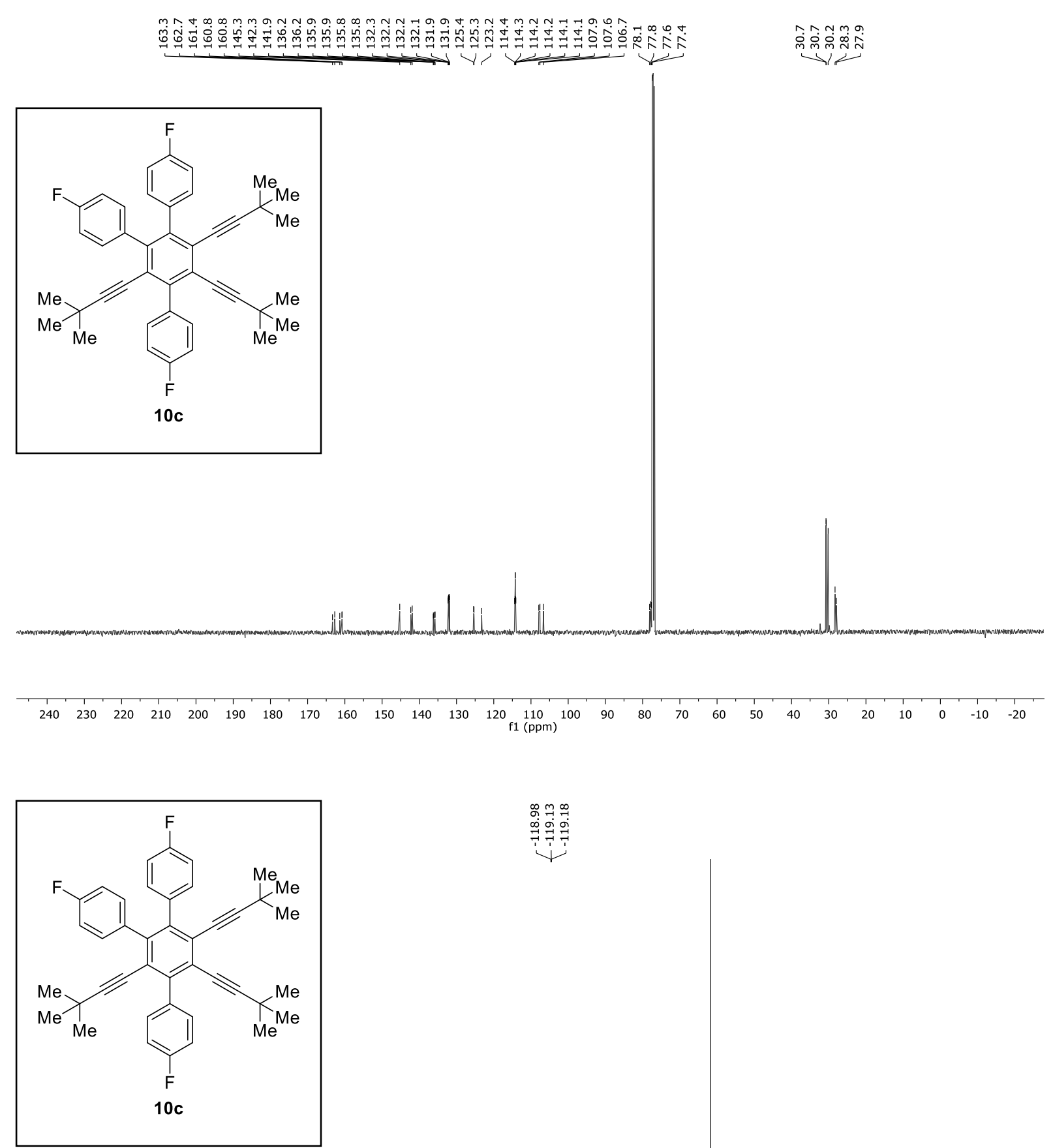

\section{क m \\ कำ}




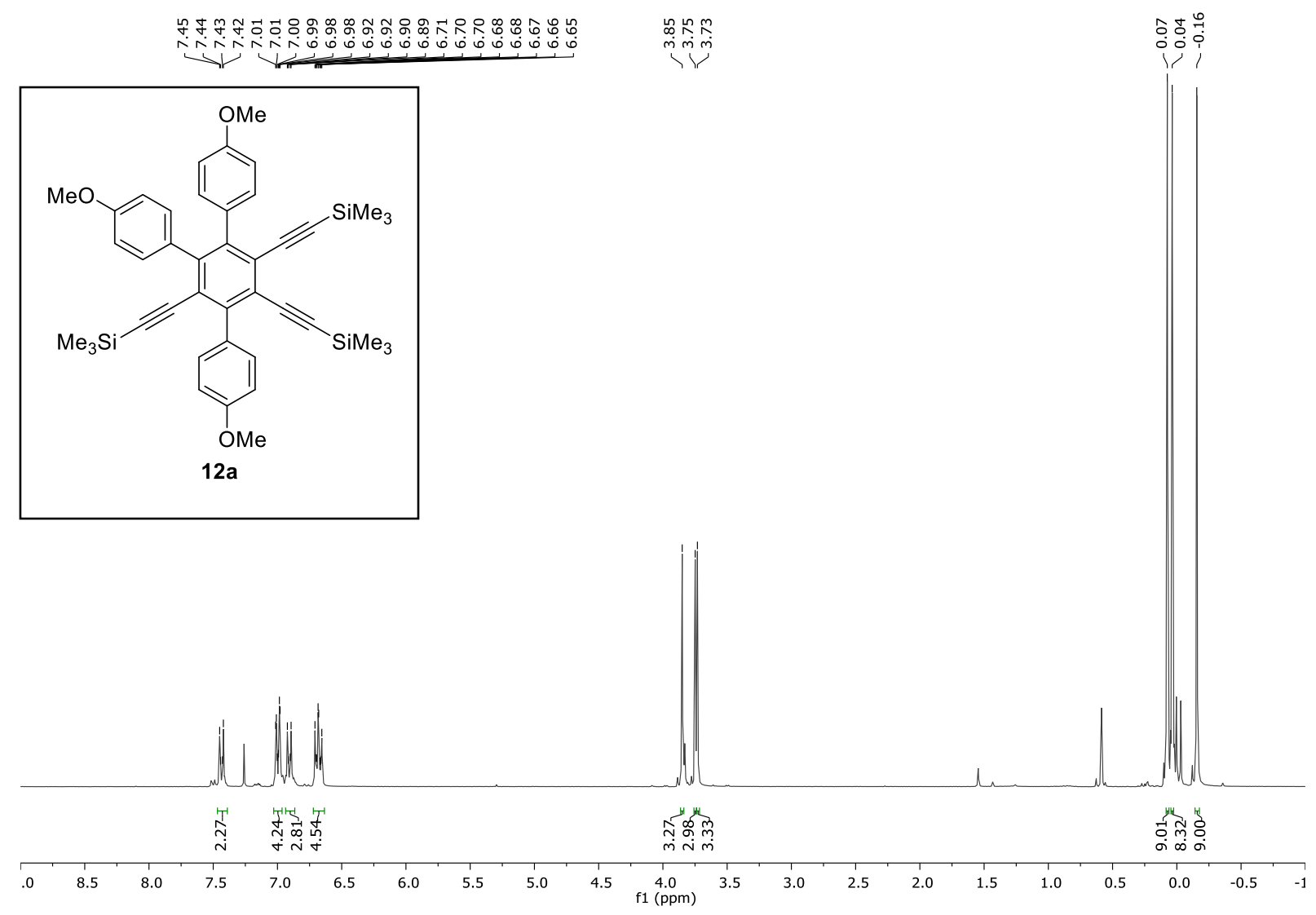

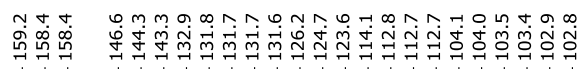

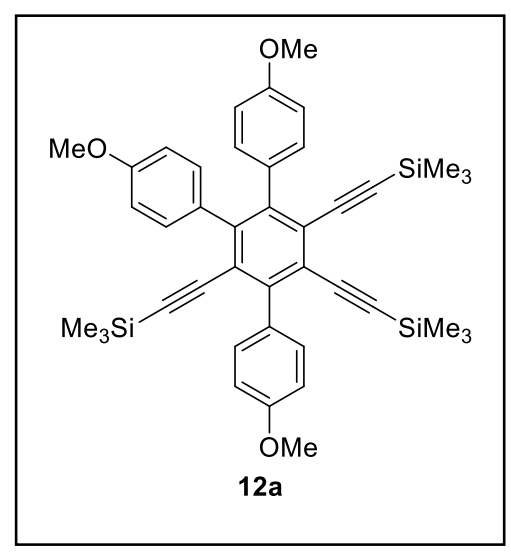

9 

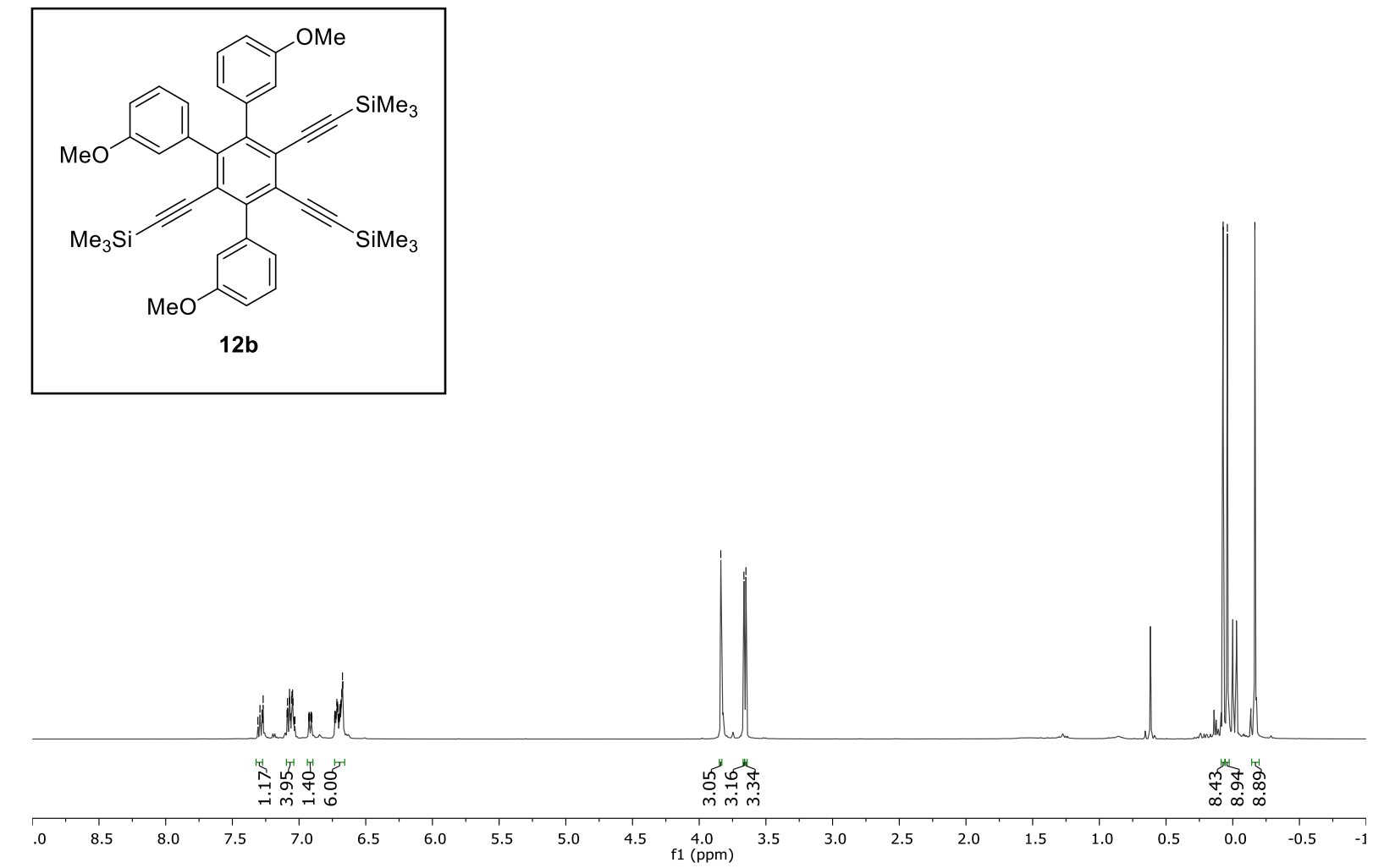

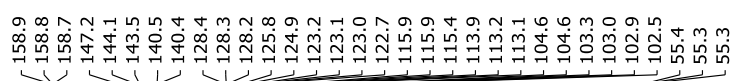
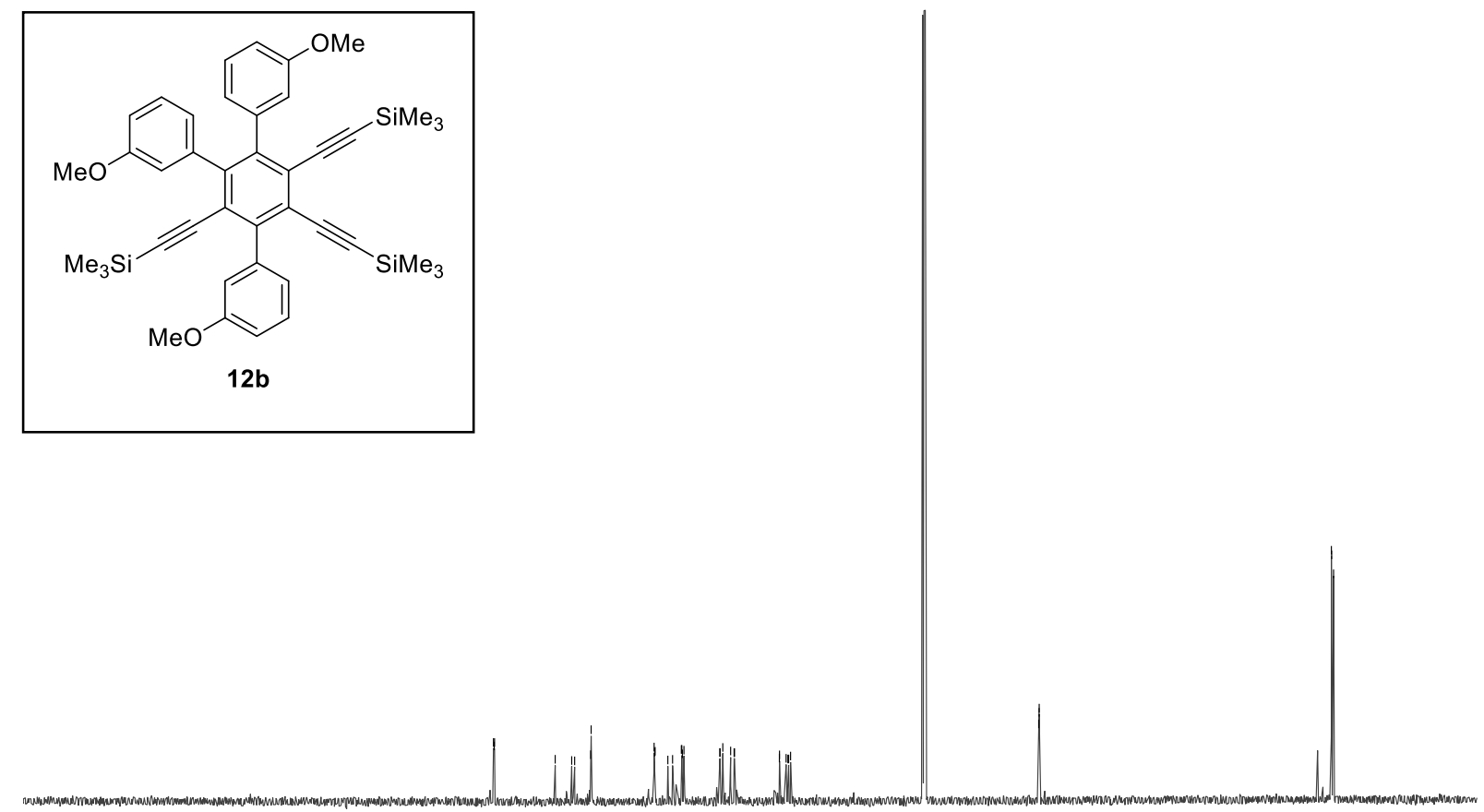

$\begin{array}{llllllllllllllllllllllllllll}240 & 230 & 220 & 210 & 200 & 190 & 180 & 170 & 160 & 150 & 140 & 130 & 120 & 110 & 100 & 90 & 80 & 70 & 60 & 50 & 40 & 30 & 20 & 10 & 0 & -10 & -20\end{array}$ 

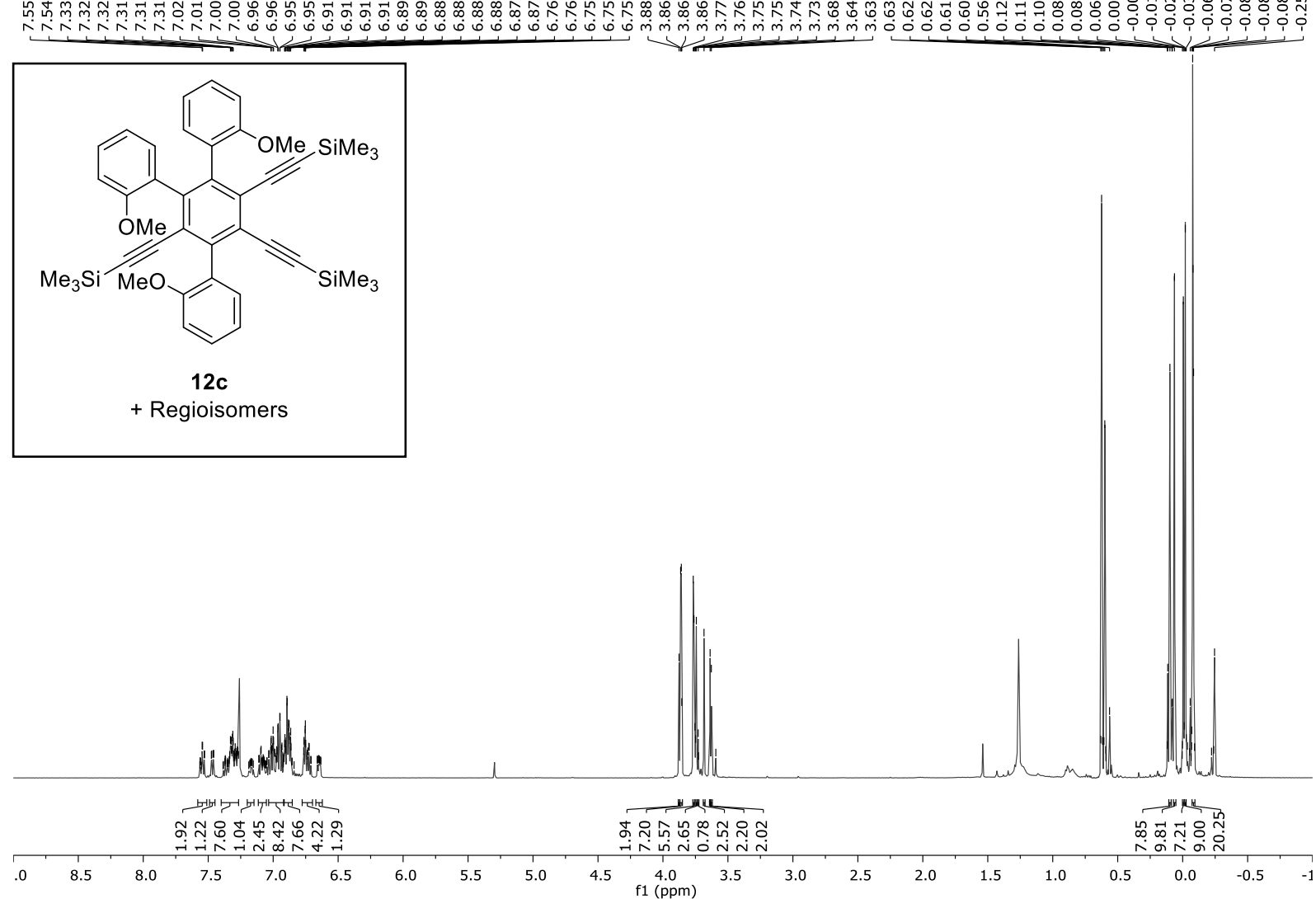

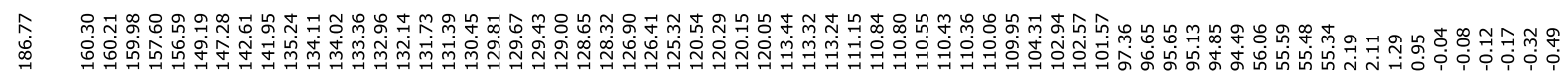

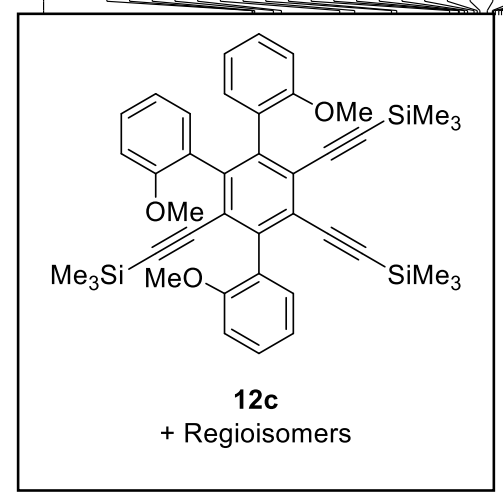

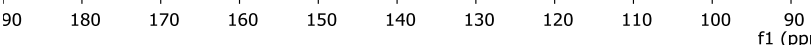



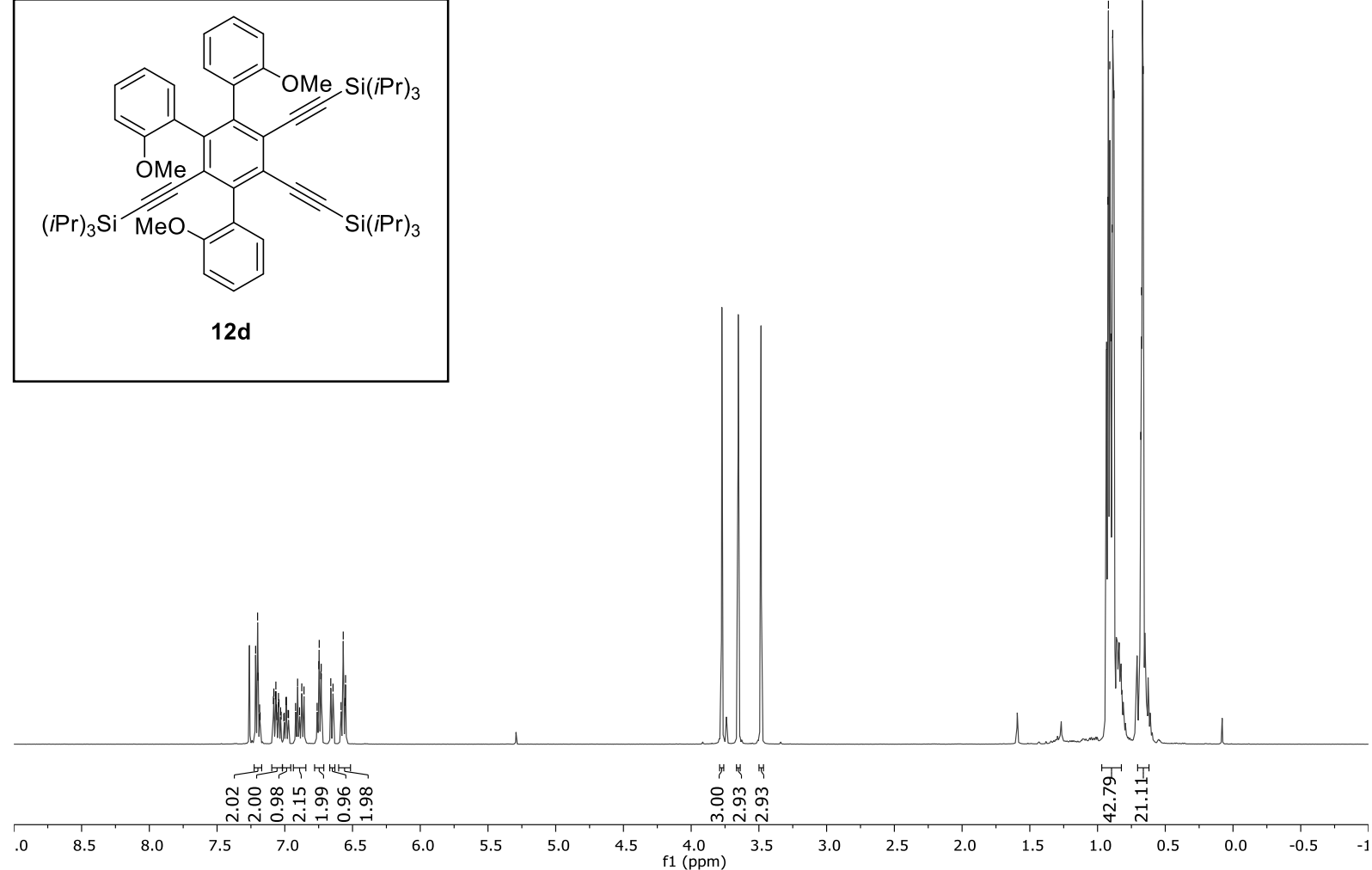

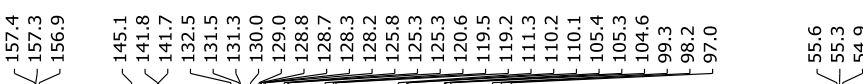

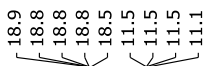
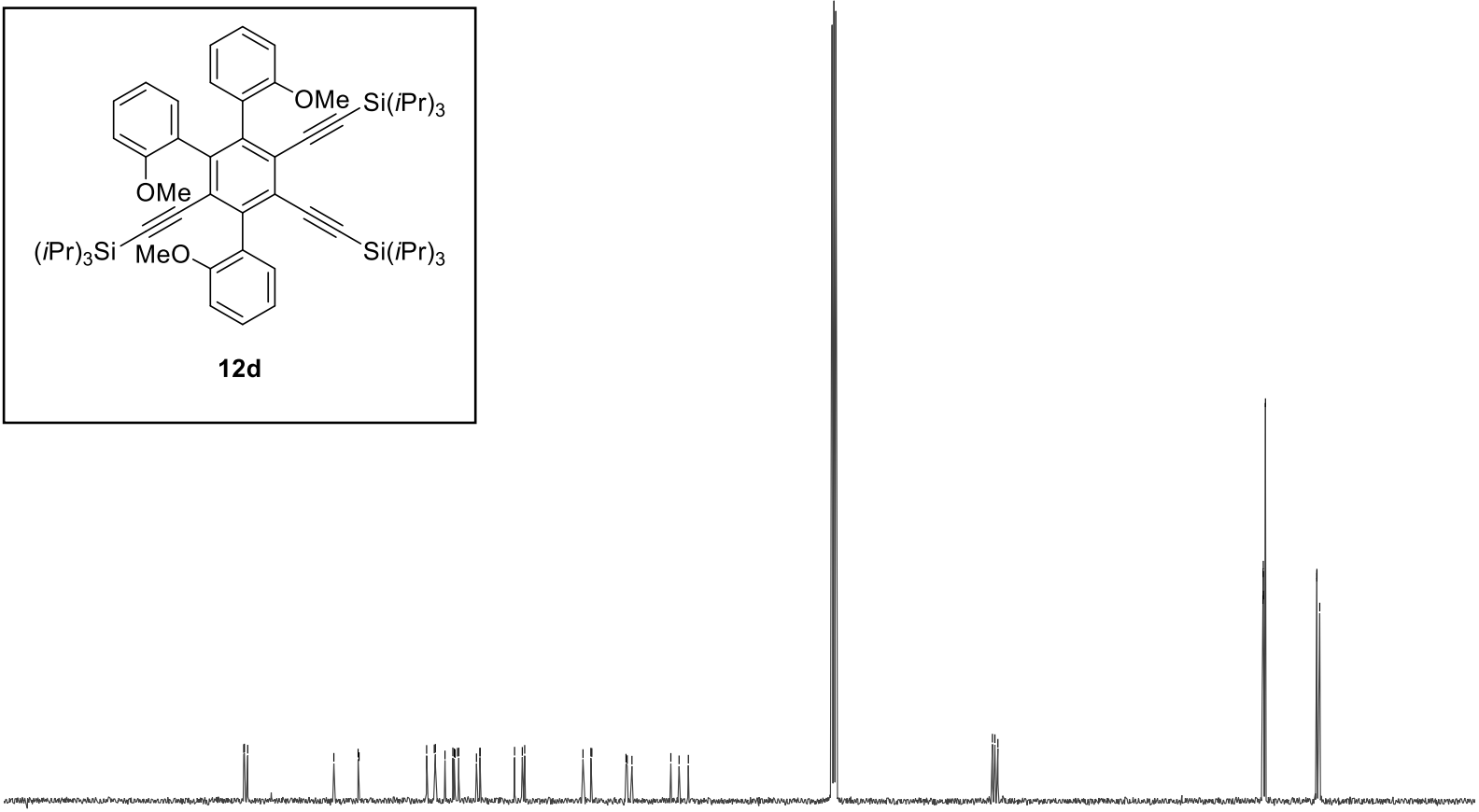

$\begin{array}{llll}130 & 120 & 110 & 100\end{array}$

f1 $\stackrel{90}{(\mathrm{ppm})}$
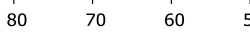

40

30 


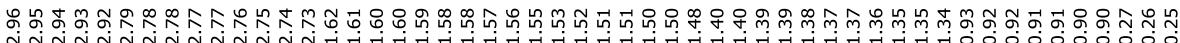

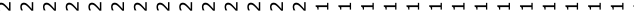
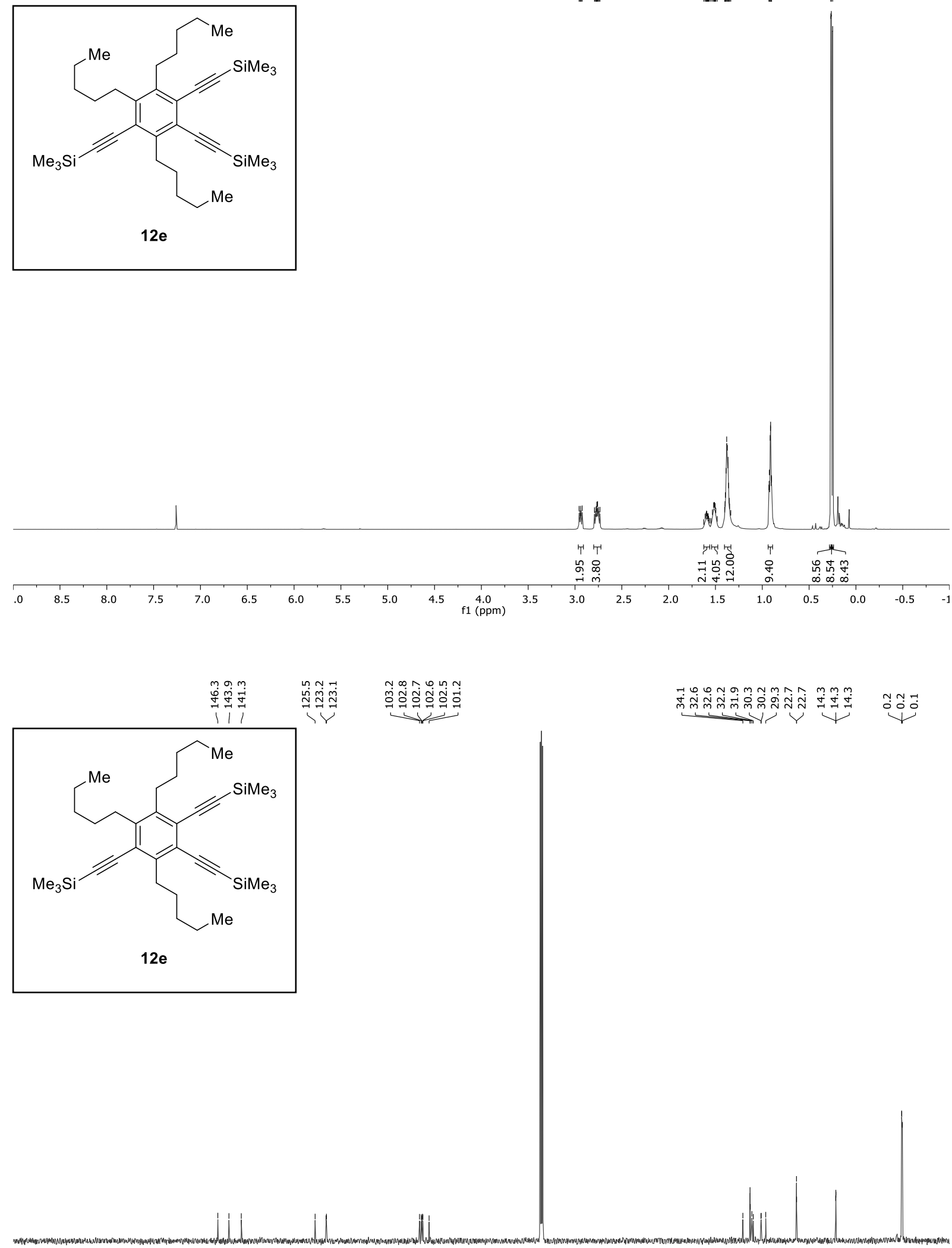

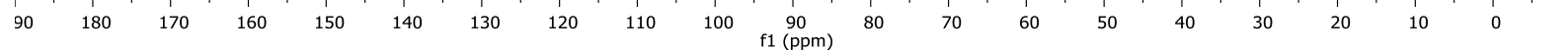




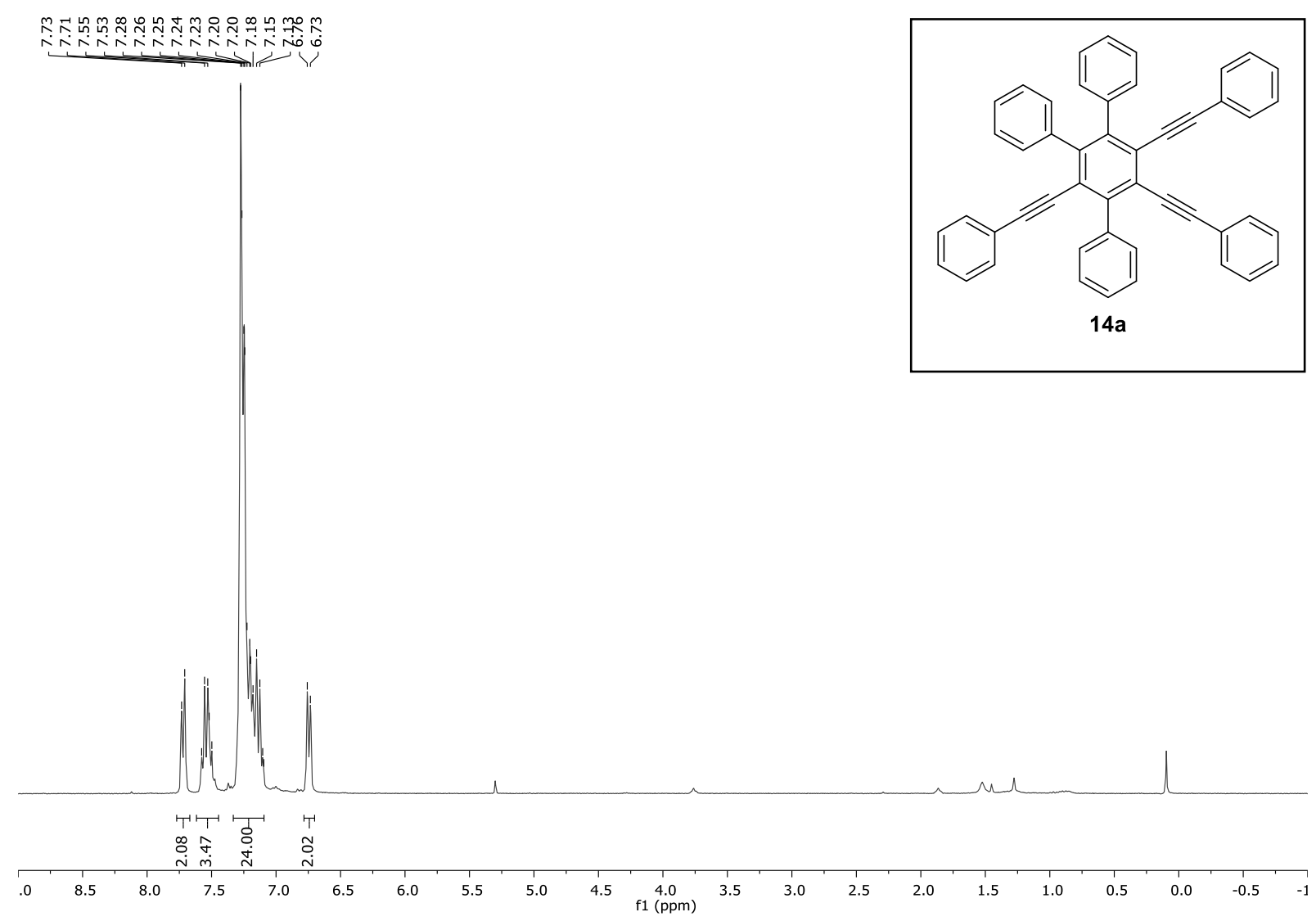

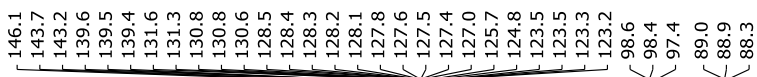
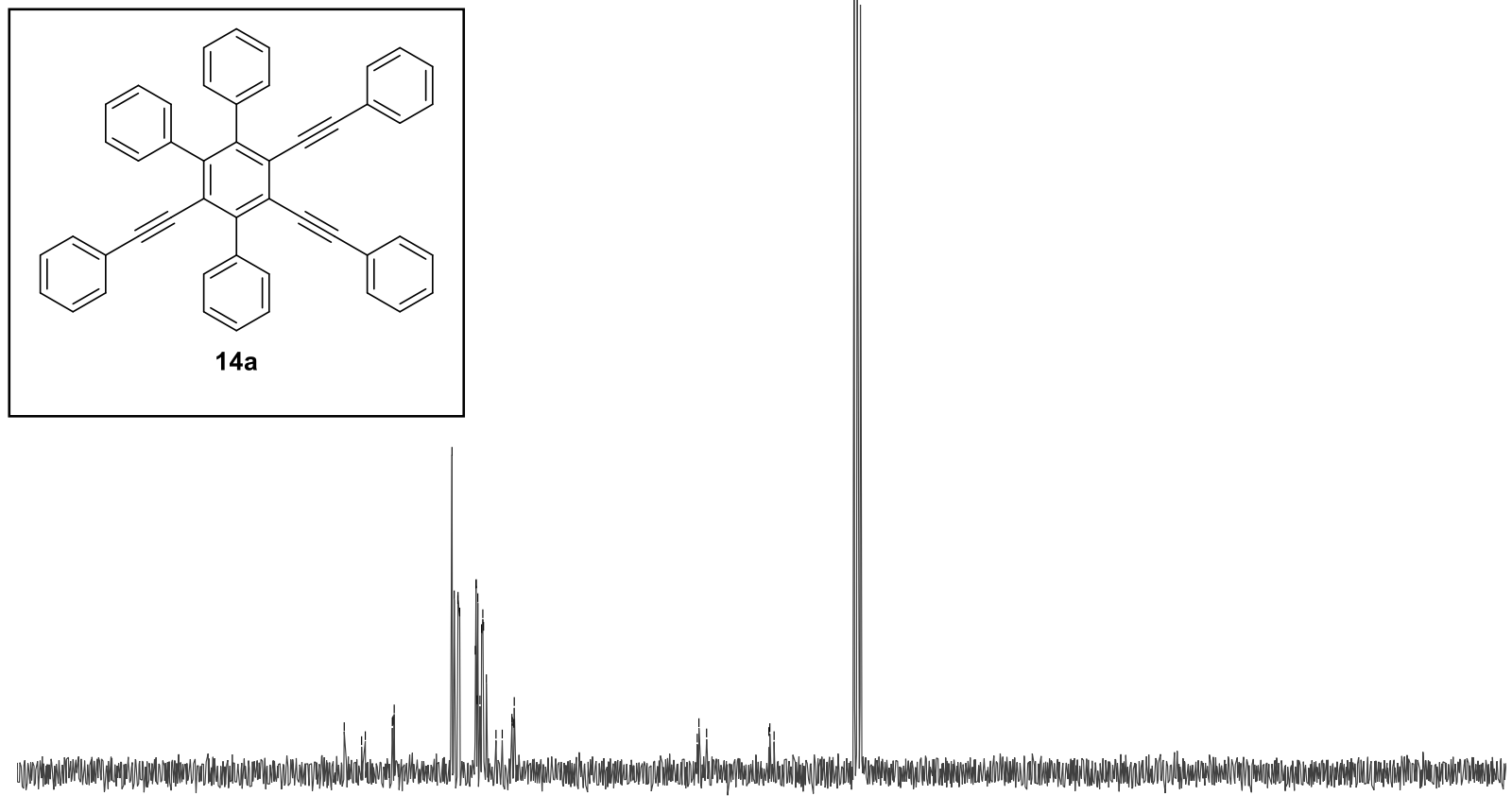

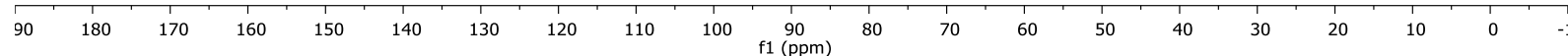




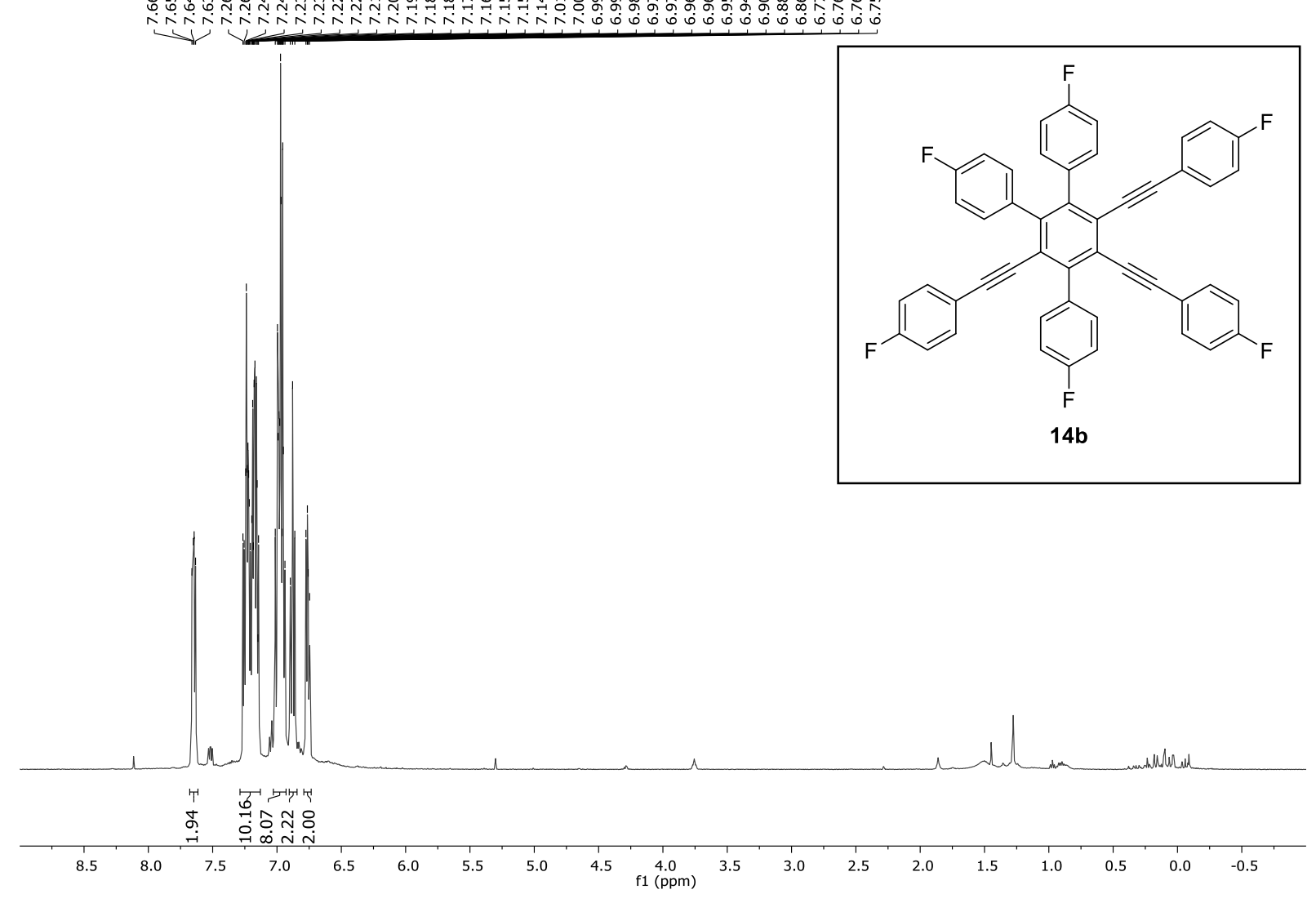

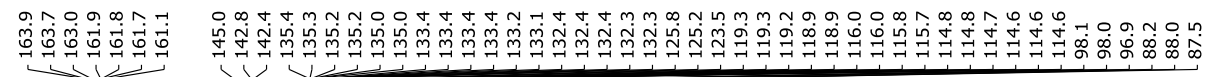
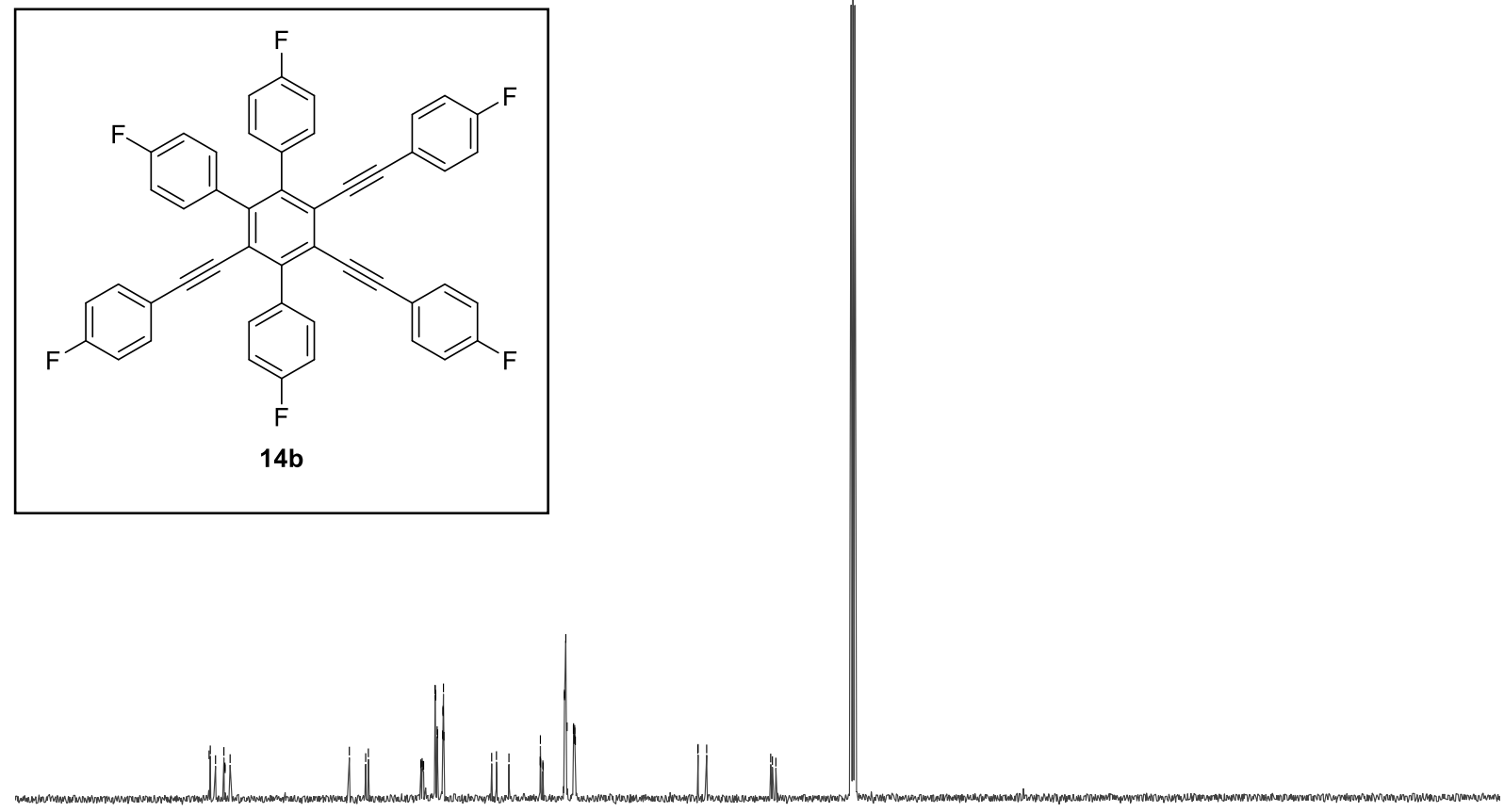

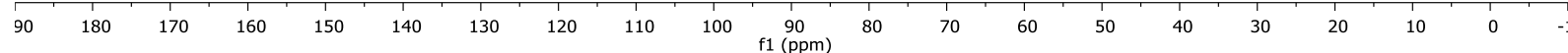




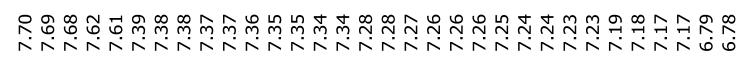

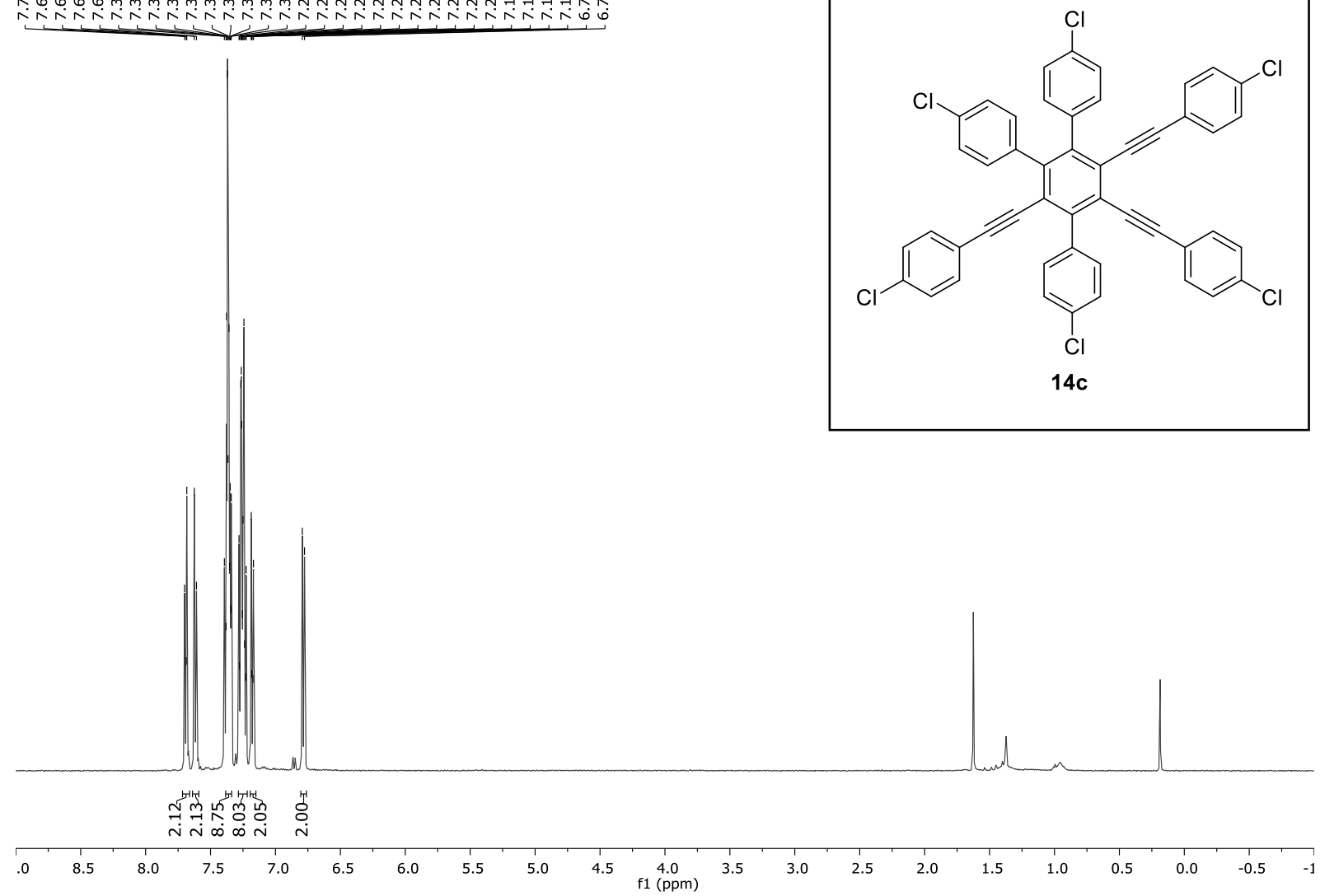

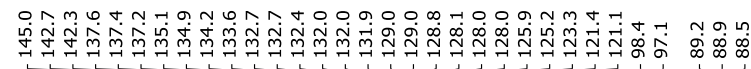

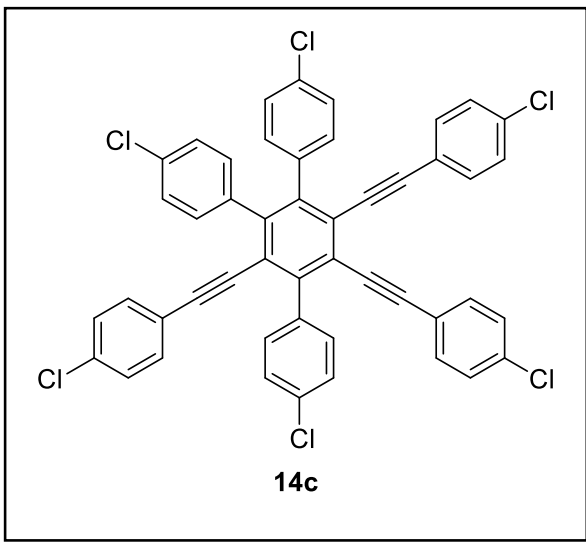

90

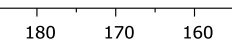

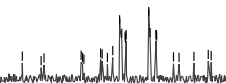

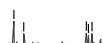



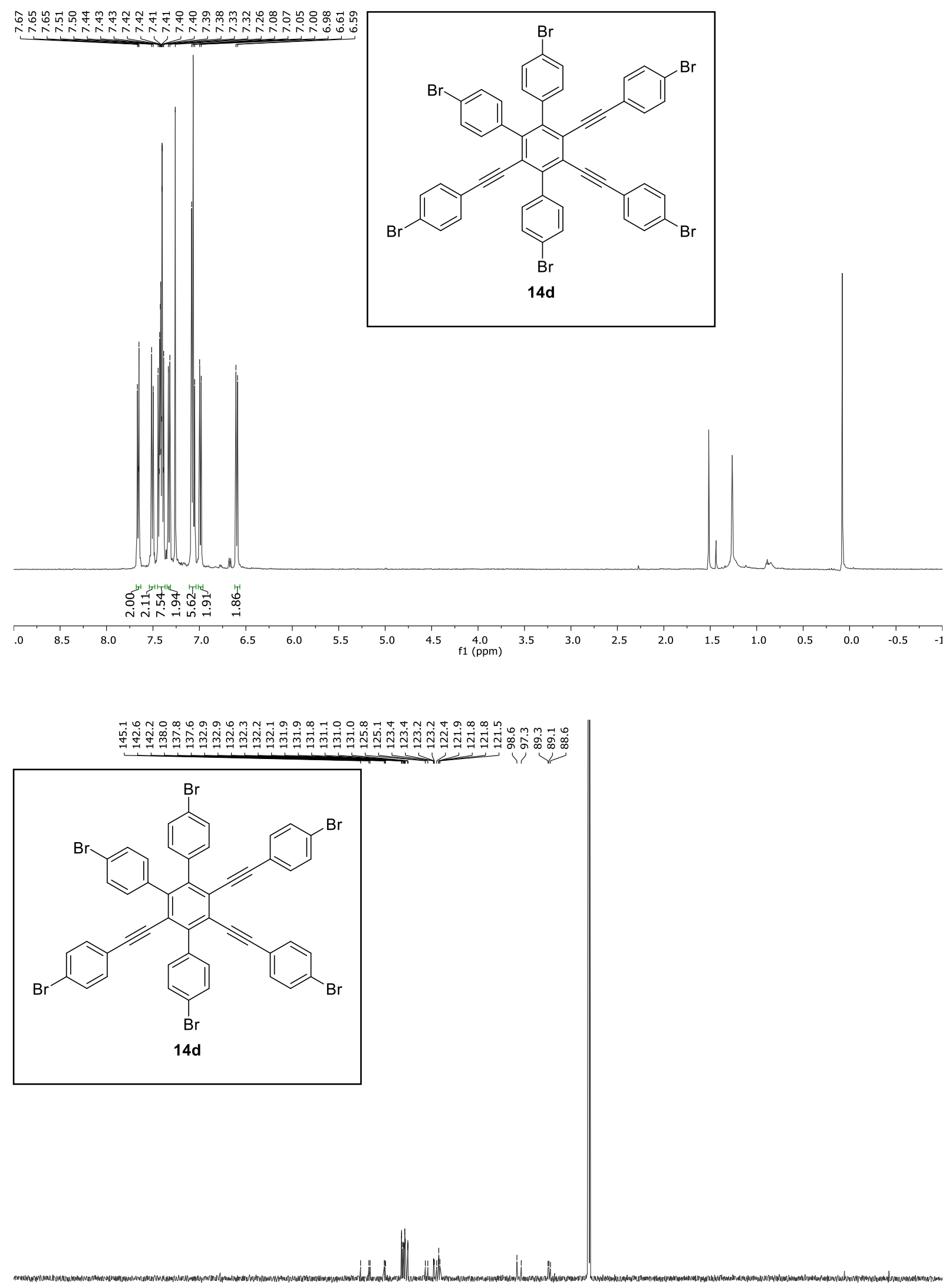

$\begin{array}{lllllllllllllllllllllllllllllllll}240 & 230 & 220 & 210 & 200 & 190 & 180 & 170 & 160 & 150 & 140 & 130 & 120 & 110 & 100 & 90 & 80 & 70 & 60 & 50 & 40 & 30 & 20 & 10 & 0 & -10 & -20\end{array}$ 


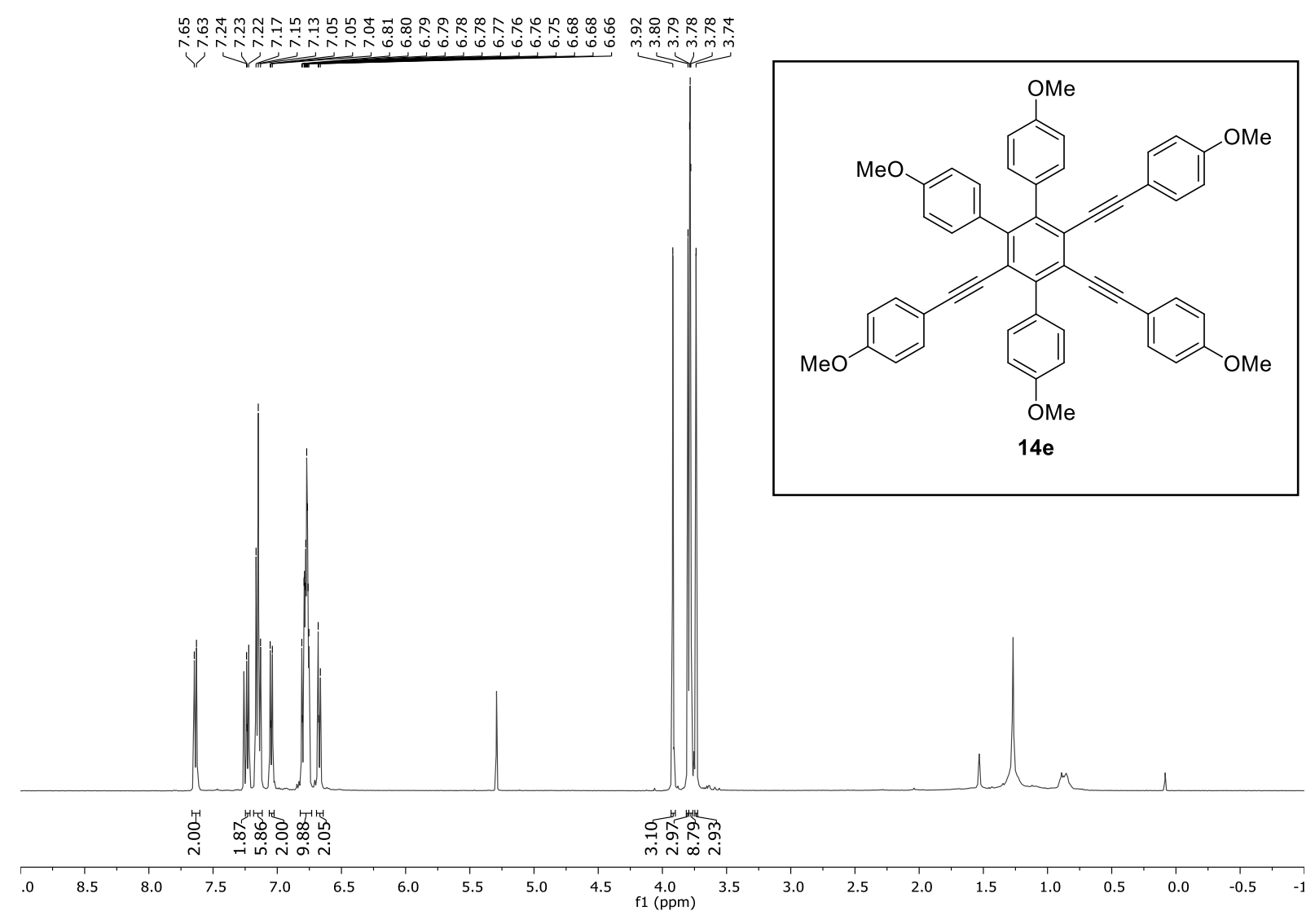

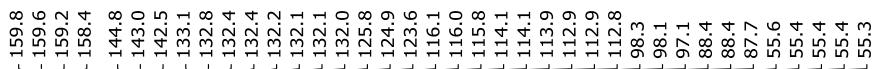

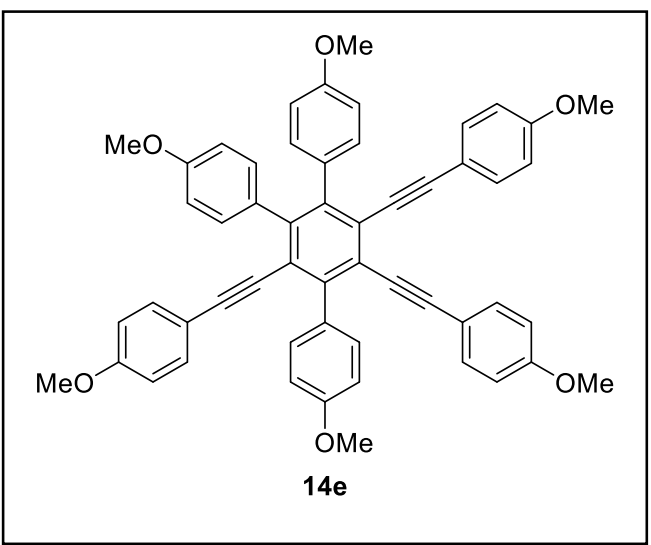

$\begin{array}{lllllllllllllllllllllllllllllllllll}240 & 230 & 220 & 210 & 200 & 190 & 180 & 170 & 160 & 150 & 140 & 130 & 120 & 110 & 100 & 90 & 80 & 70 & 60 & 50 & 40 & 30 & 20 & 10 & 0 & -10 & -20\end{array}$ 


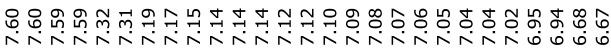

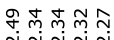
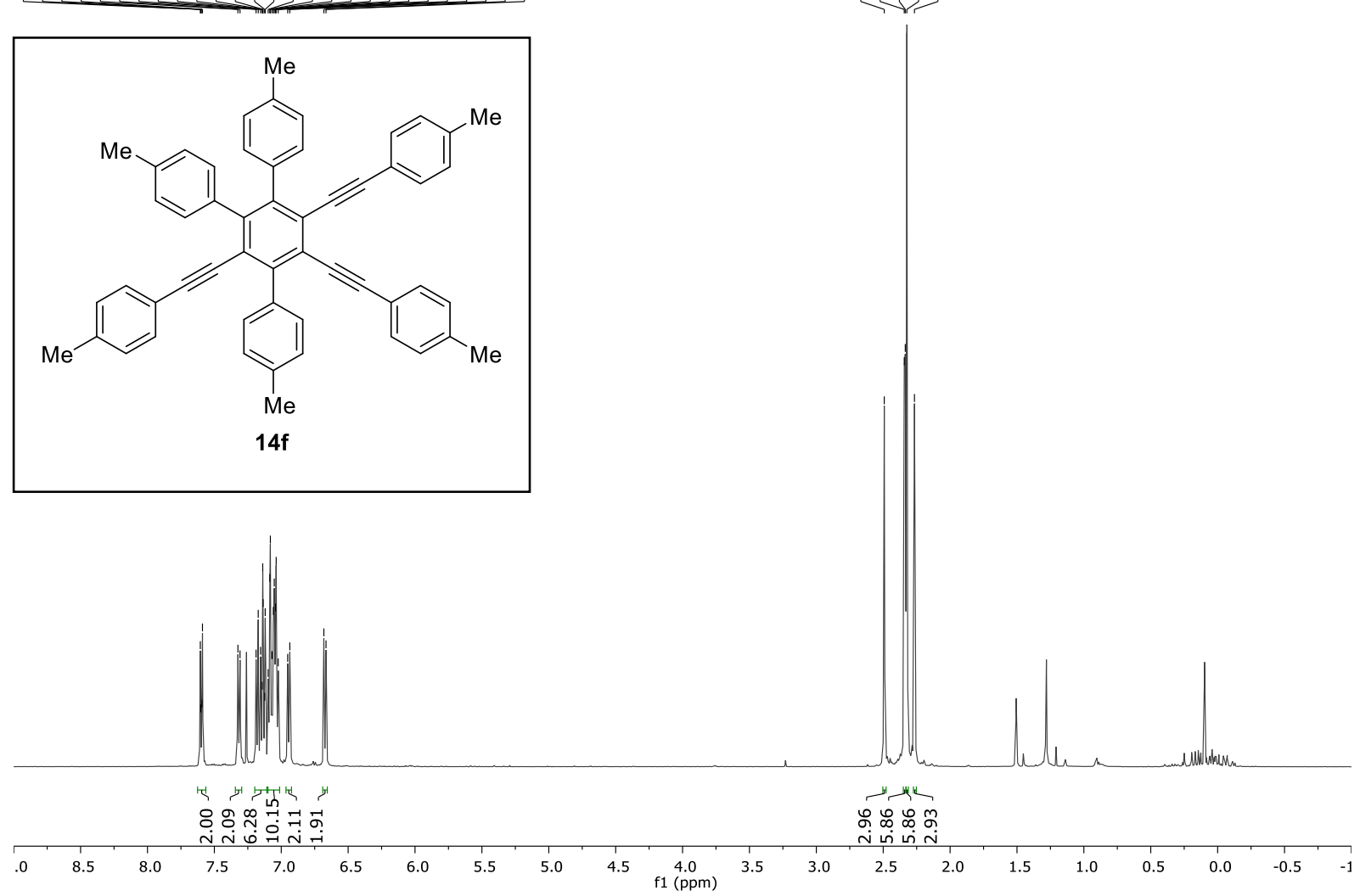

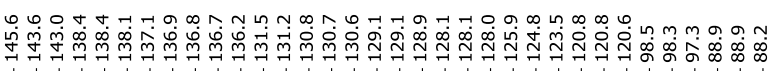

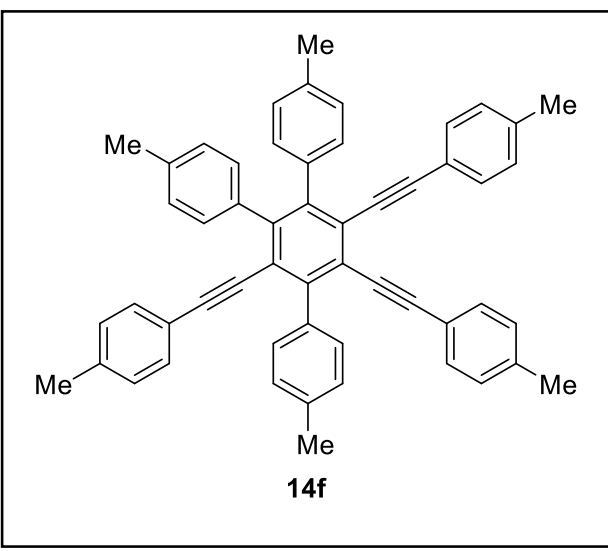

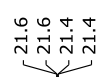

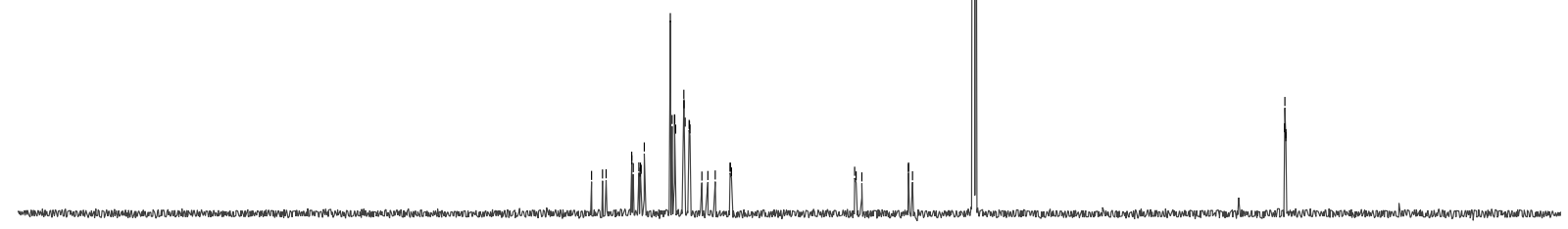

$\begin{array}{lllllllllllllllllllllllllllll}240 & 230 & 220 & 210 & 200 & 190 & 180 & 170 & 160 & 150 & 140 & 130 & 120 & 110 & 100 & 90 & 80 & 70 & 60 & 50 & 40 & 30 & 20 & 10 & 0 & -10 & -20\end{array}$ 


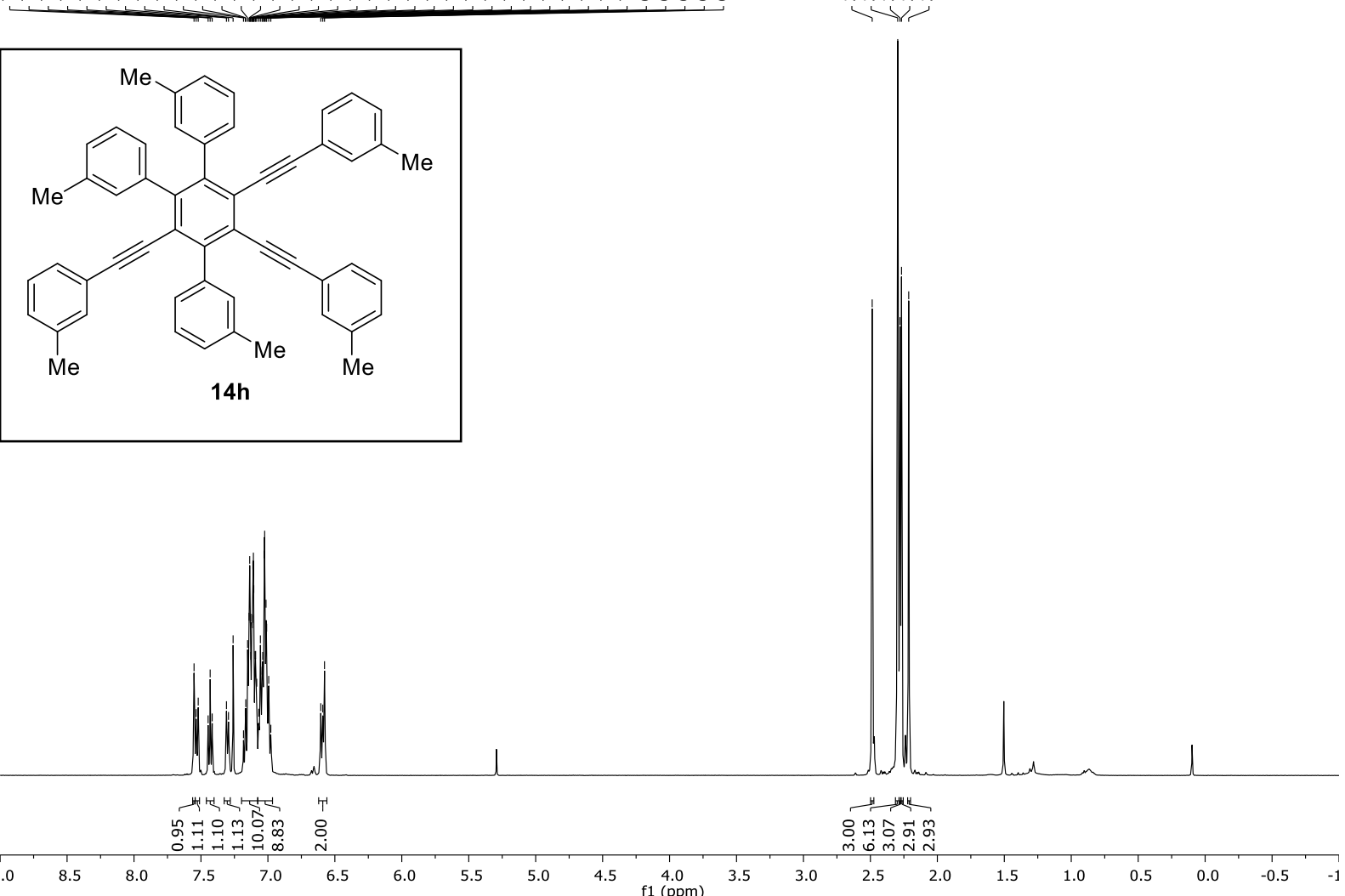

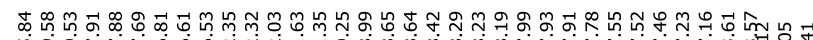

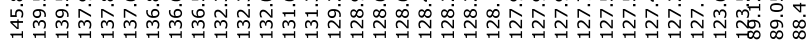

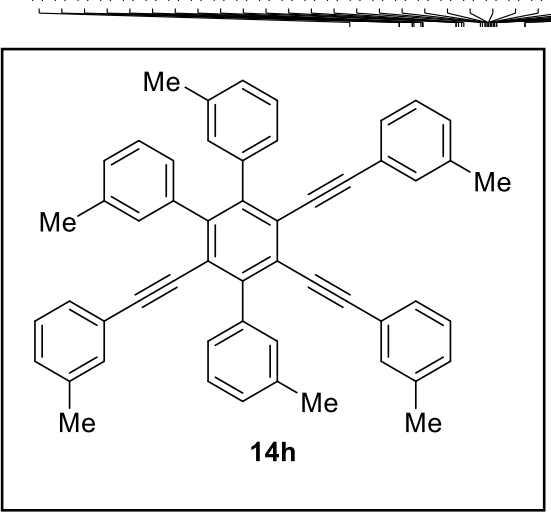

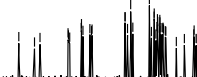

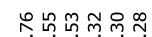

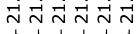

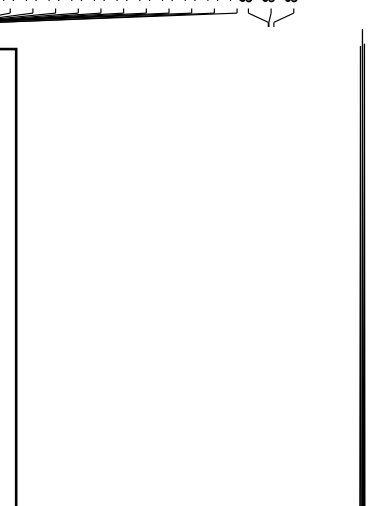

$\begin{array}{llllllllllllllllllllll}90 & 180 & 170 & 160 & 150 & 140 & 130 & 120 & 110 & 100 & \underset{\mathrm{f} 1(\mathrm{ppm})}{90} & 80 & 70 & 60 & 50 & 40 & 30 & 20 & 10 & 0 & -\end{array}$ 

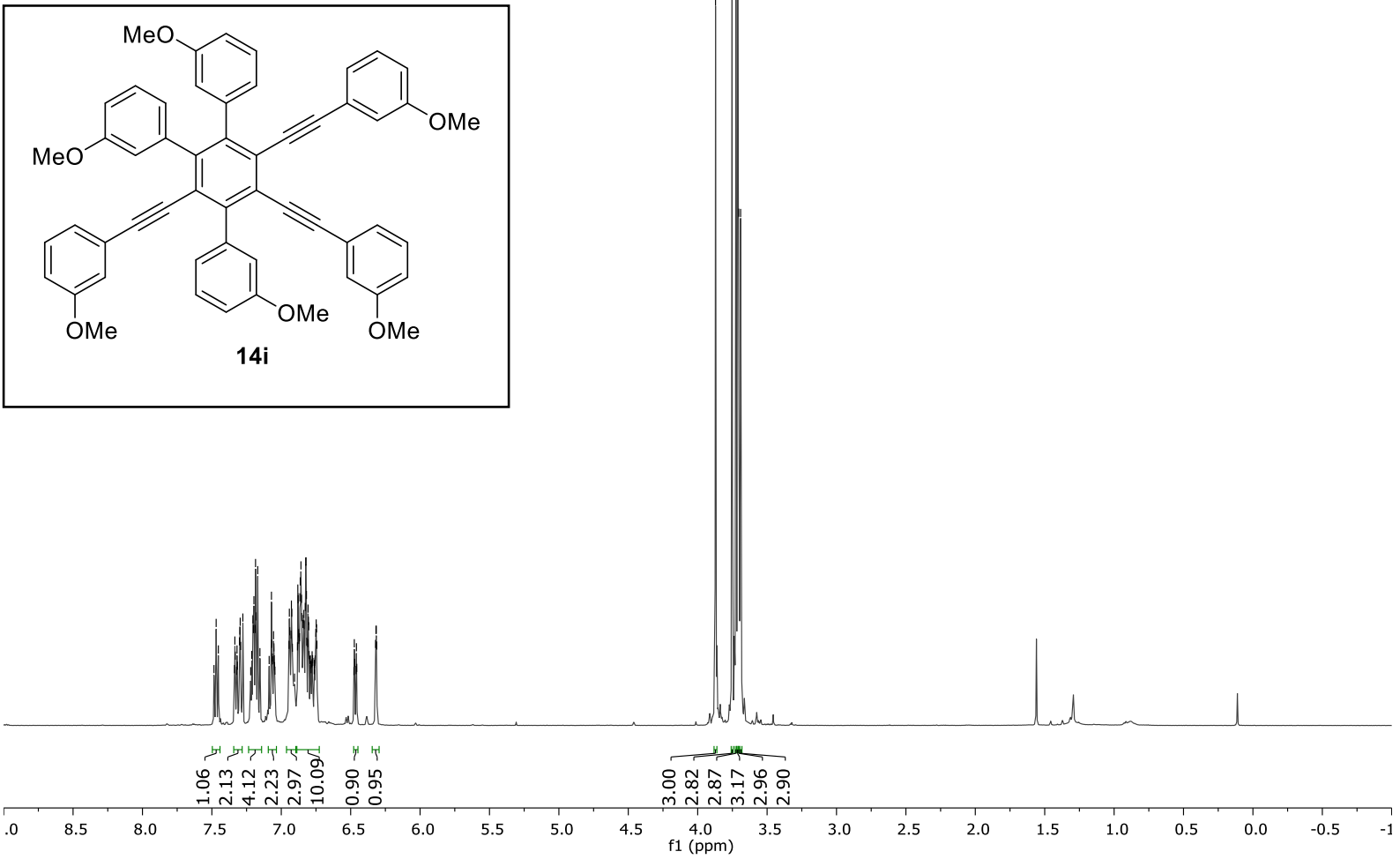

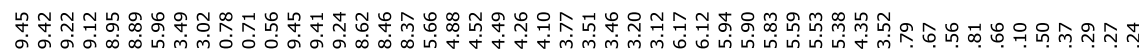

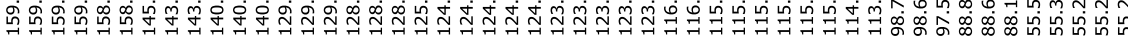

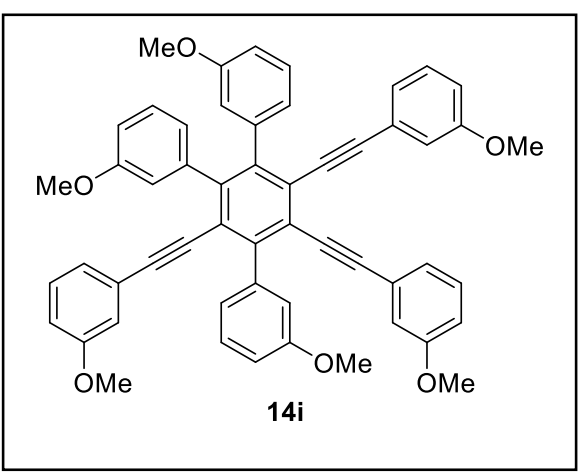

90

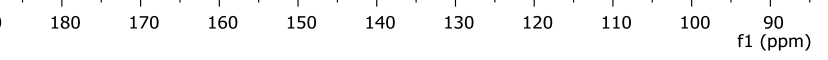
80 50 $40 \quad 30$ $20 \quad 10 \quad 0 \quad-$ 


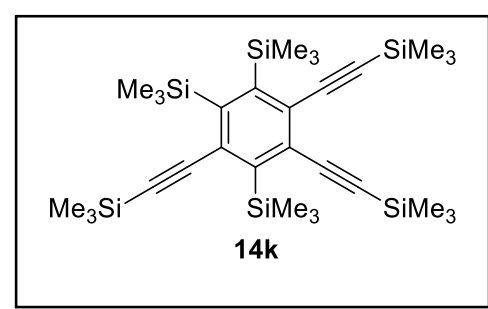

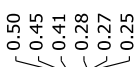

|

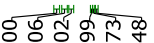

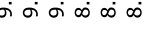

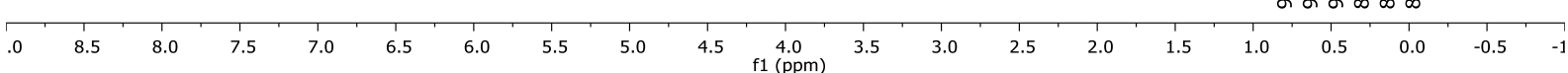

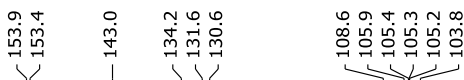
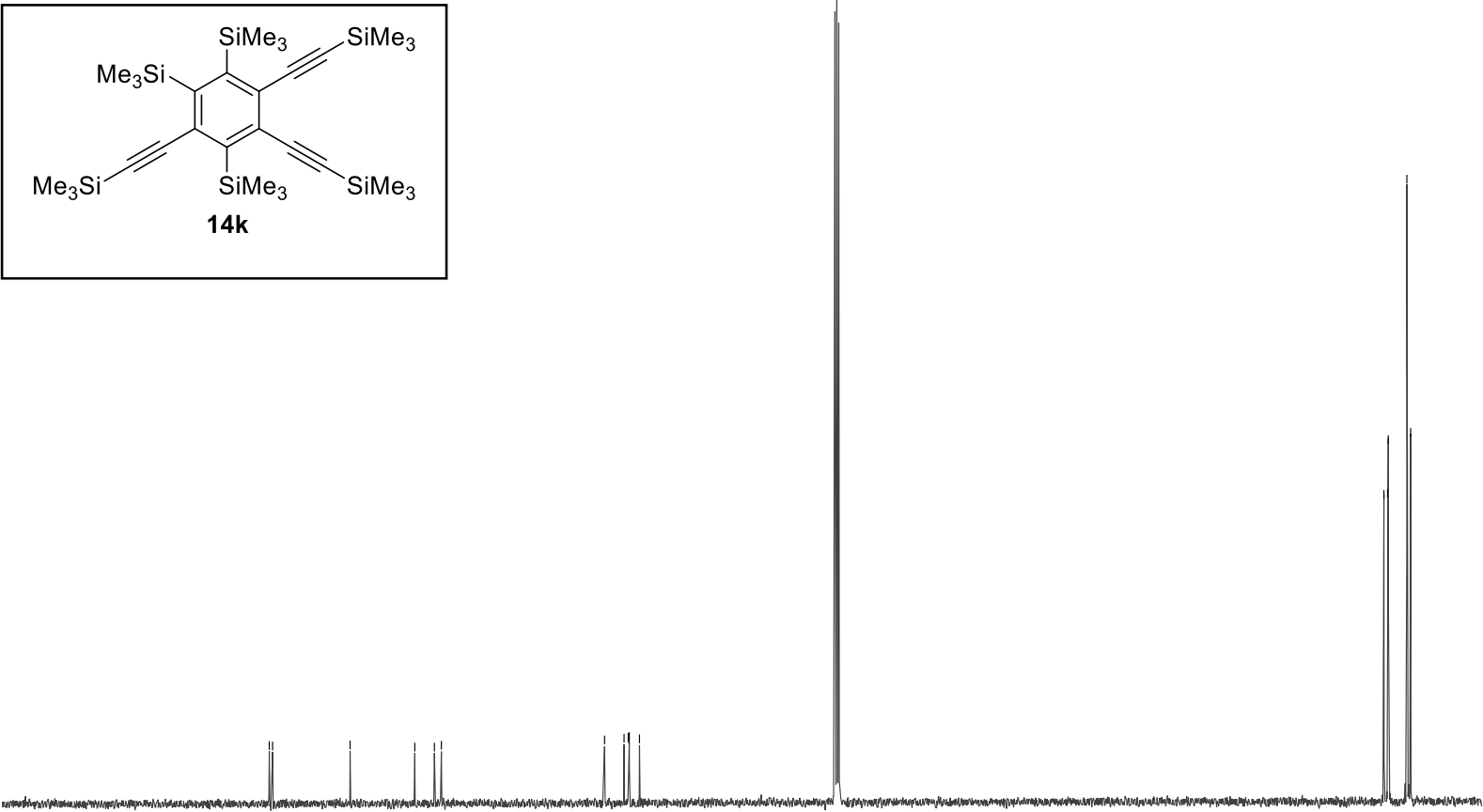

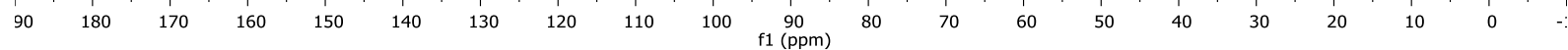




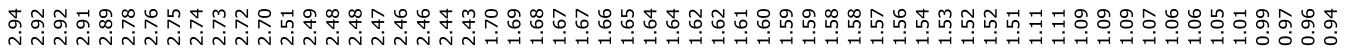

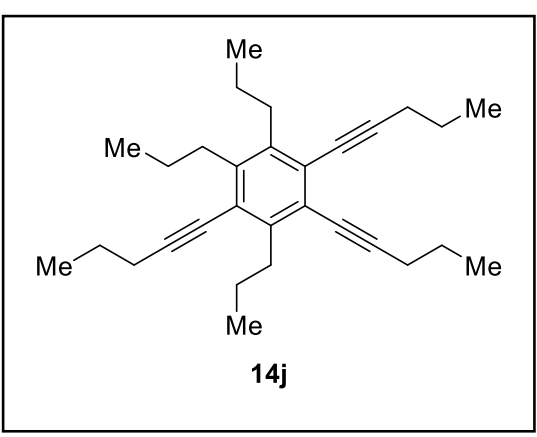

$14 \mathrm{j}$
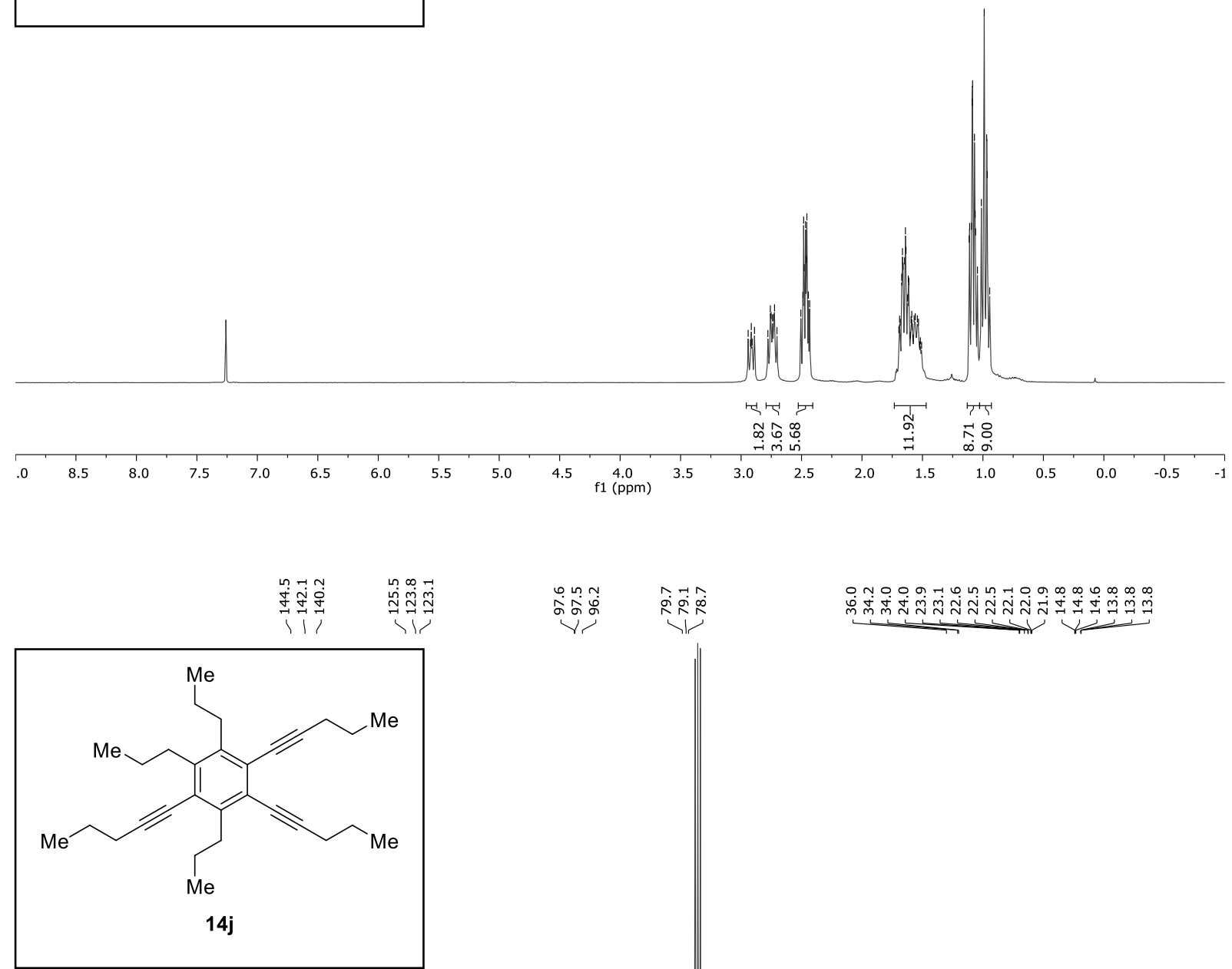

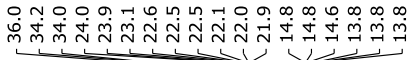

\|\|$_{\|} \quad \| \mid$

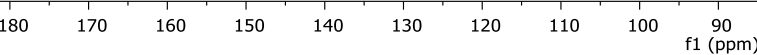

$80 \quad 70$

60

50

$40 \quad 30$

$20 \quad 10 \quad 0 \quad-:$ 


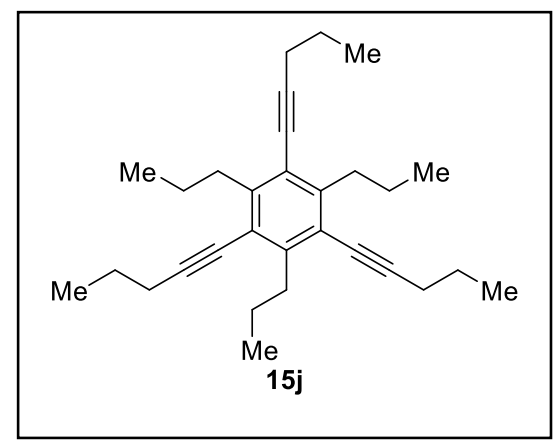

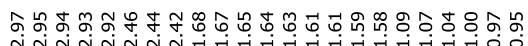

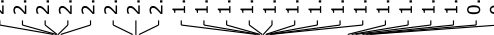
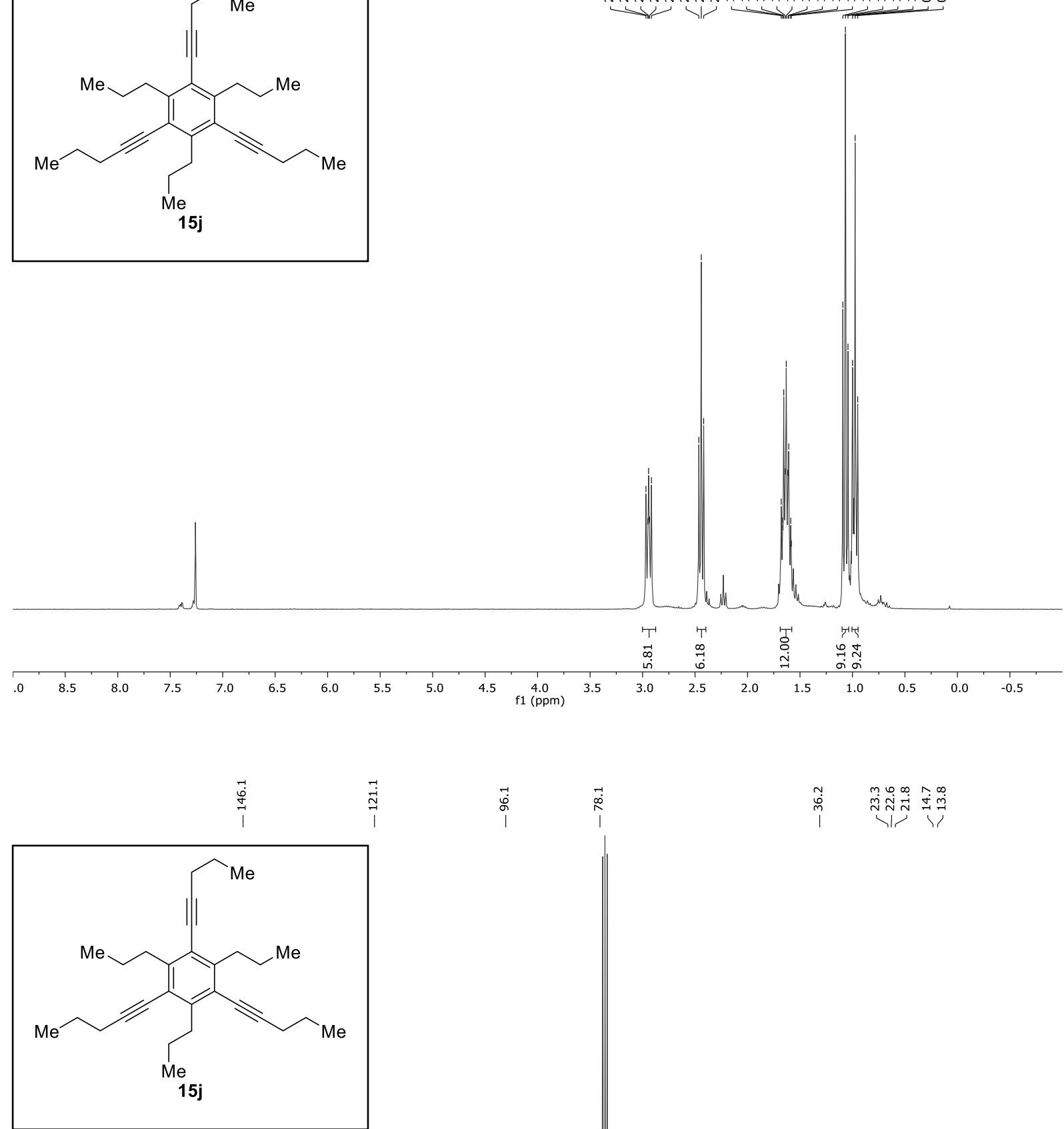

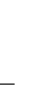




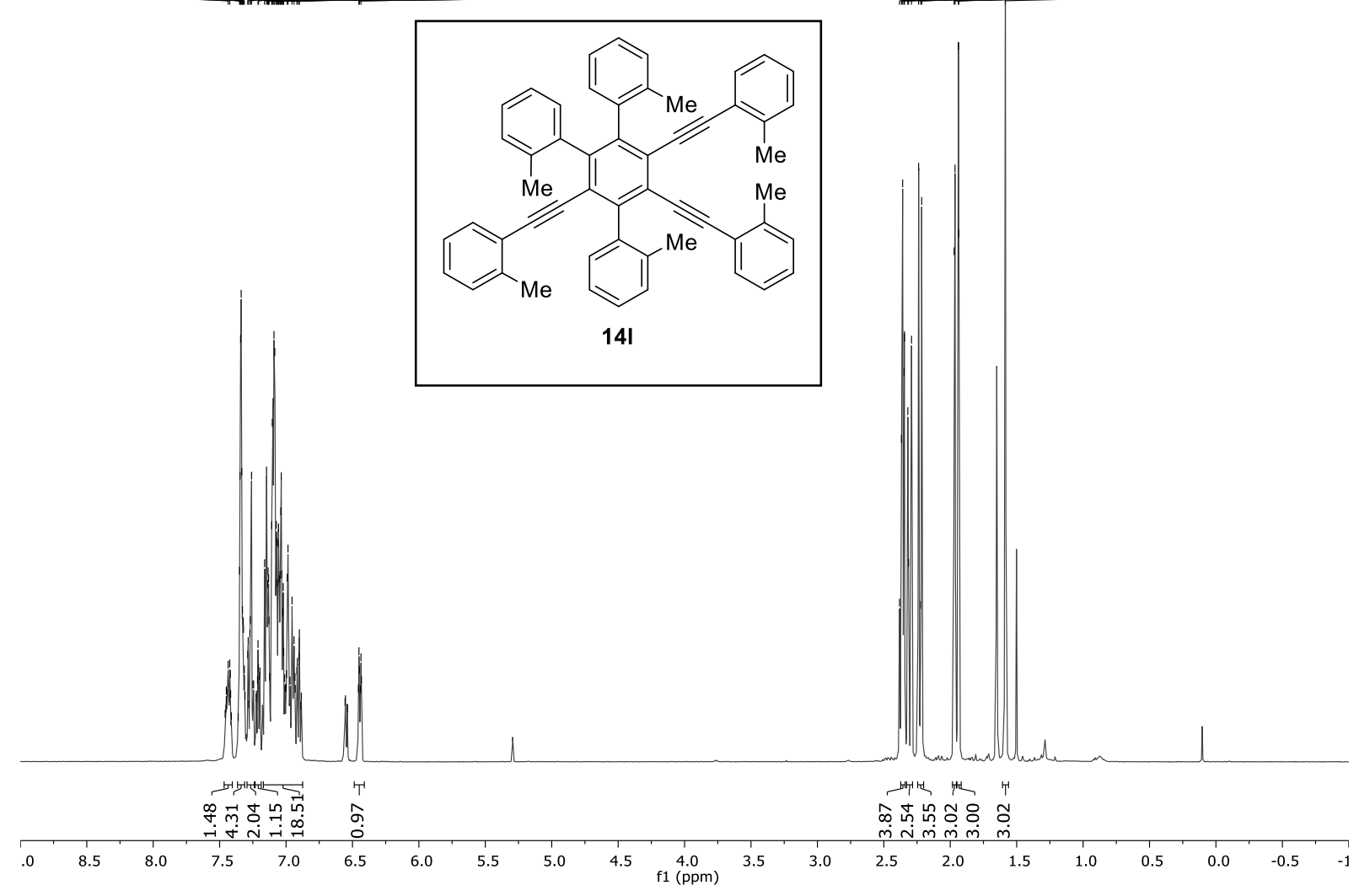

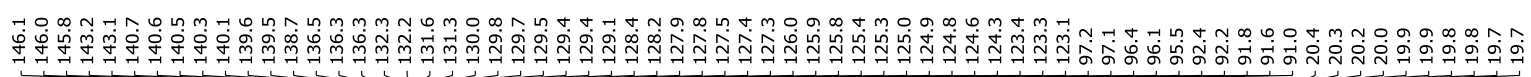

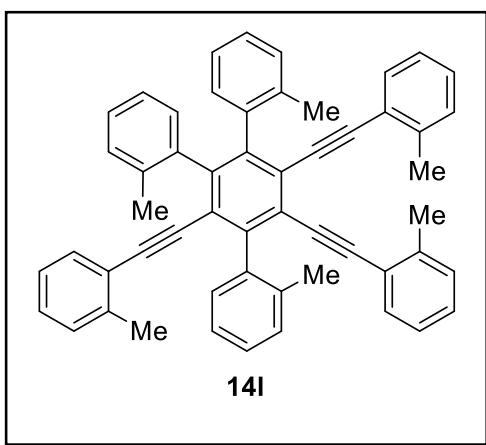

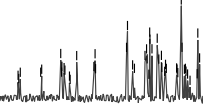
in

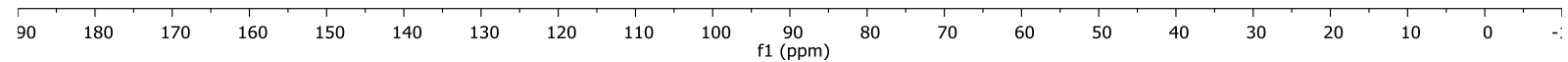




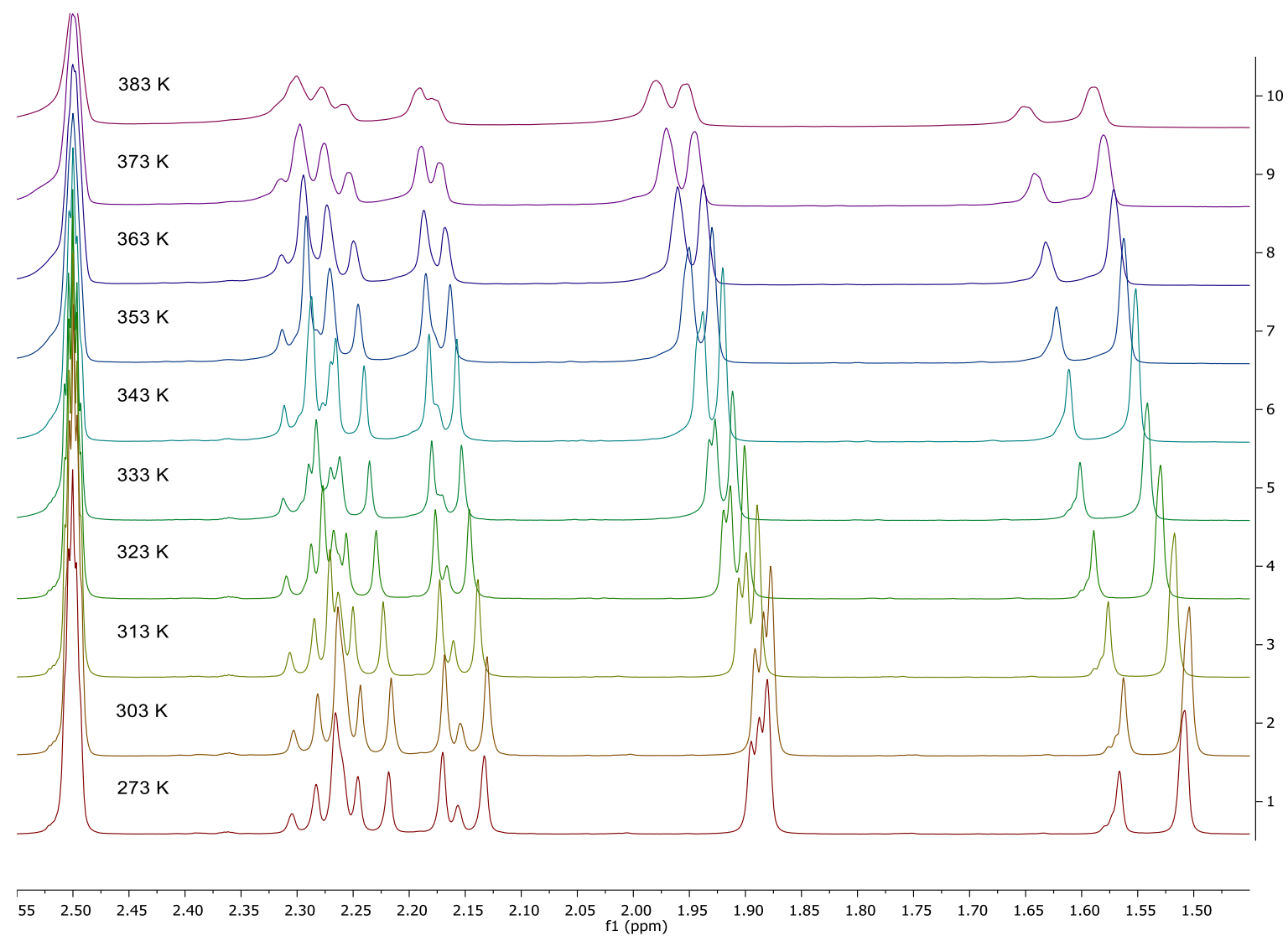

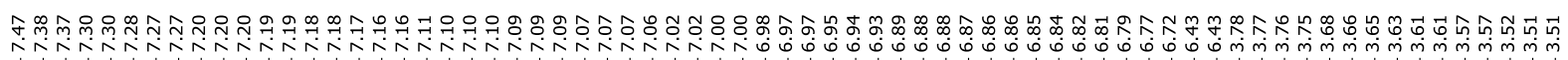
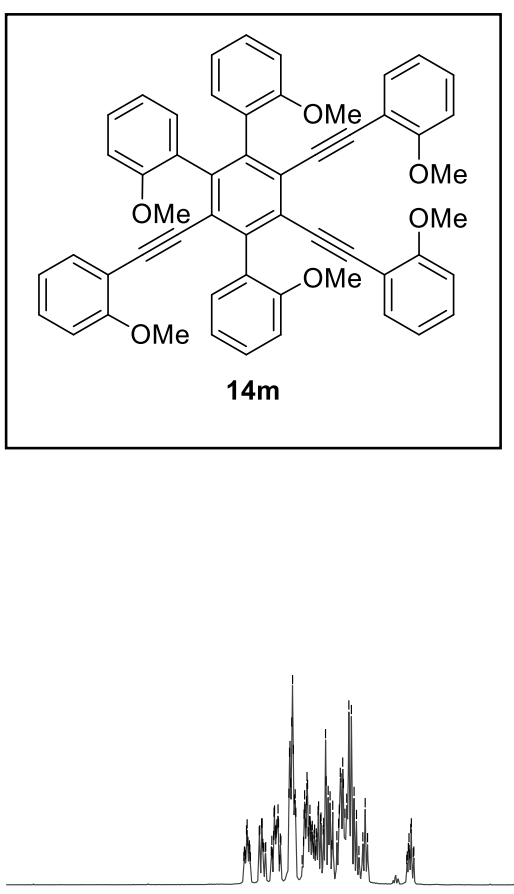

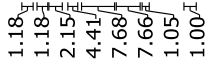

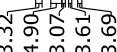

\begin{tabular}{lllllllllllllllllllll}
\hline .0 & 8.5 & 8.0 & 7.5 & 7.0 & 6.5 & 6.0 & 5.5 & 5.0 & 4.5 & $\begin{array}{c}1 \\
\mathrm{f} 1\end{array}(\mathrm{ppm})$ & 3.5 & 3.0 & 2.5 & 2.0 & 1.5 & 1.0 & 0.5 & 0.0 & -0.5 & -1
\end{tabular}




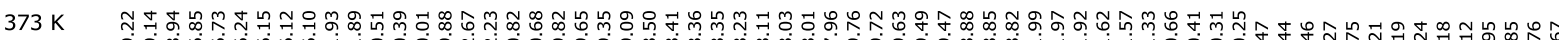

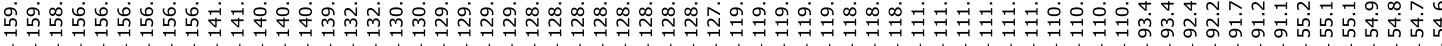

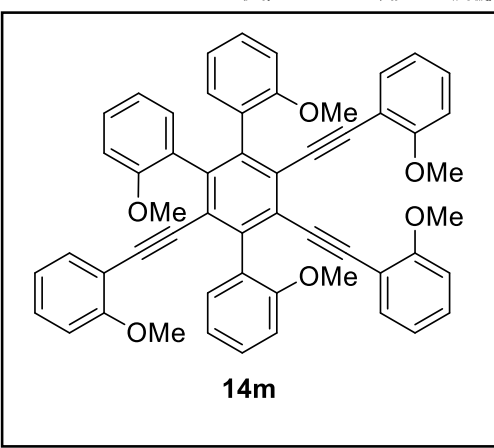

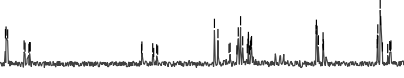
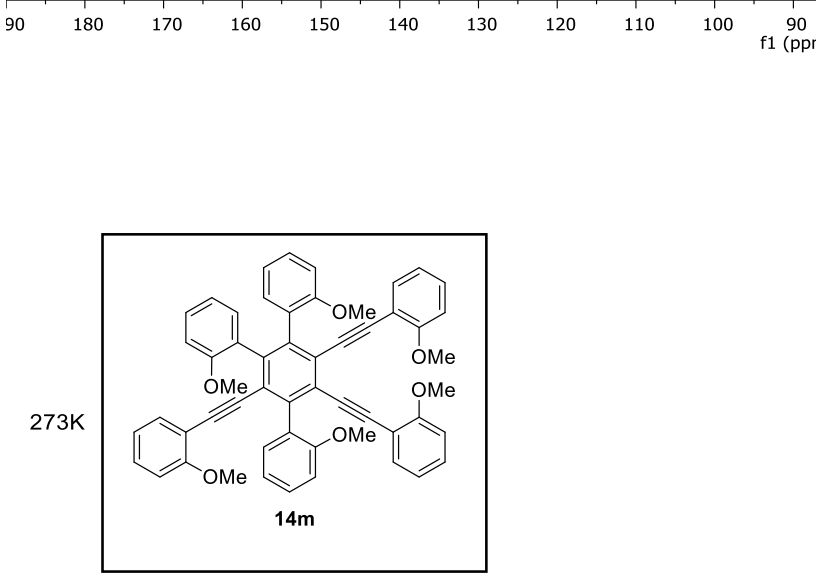

$M$

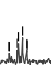

$\mathrm{f} 1(\mathrm{ppm})$

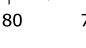

$373 \mathrm{~K}$

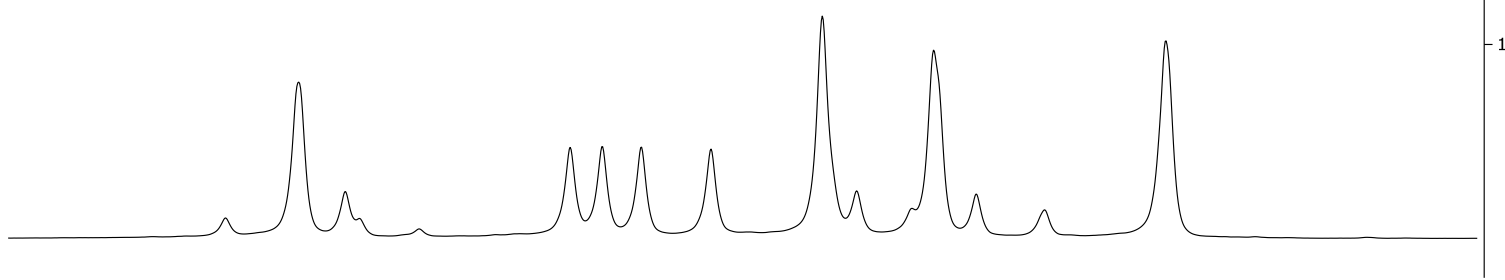

3.85

3.80

3.75

3.70

3.65
$f 1(\mathrm{ppm})$

3.55

$3.50 \quad 3.45$ 


\section{References}

[1] Hilt, G.; Lüers, S. Synthesis 2002, 609-618.

[2] Weber, S. M.; Hilt, G. Org. Lett. 2017, 19, 564-567.

[3] Röse, P.; Garcia, C. C. M.; Pünner, F.; Harms, K.; Hilt, G. J. Org. Chem. 2005, 80, 7311-7316.

[4] Schmidt, R.; Thorwirth, R.; Szuppa, T.; Stolle, A.; Ondruschka, B.; Hopf, H. Chem. Eur. J. 2011, 17, 81298138.

[5] Merkul, E.; Urselmann, D.; Müller, T. J. J. Eur. J. Org. Chem. 2011, 2, 238-242.

[6] Hoheisel, T. N.; Frauenrath, H. Org. Lett. 2008, 16, 4467-4470.

[7] Fairlamb, I. J. S.; Bäuerlein, P. S.; Marrison, L. R.; Dickinson, J. M. Chem. Commun. 2003, 632-633.

[8] Adimurthy, S.; Malakar, C. C.; Beifuss, U. J. Org. Chem. 2009, 74, 5648 -5651.

[9] Kamata, K.; Yamaguchi, S.; Kotani, M.; Yamaguchi, K.; Mizuno, N. Angew. Chem. Int. Ed. 2008, 47, 24072410.

[10] Lei, A.; Yin, W.; Chen, M.; He, C.; Zhang, H. Org. Lett. 2008, 11, 709-712.

[11] Ravera, M.; D'Amato, R.; Guerri, A. J. Organomet. Chem. 2005, 2376-2380.

[12] Shukla, R.; Lindeman, S. V.; Rathore, R. Org. Lett. 2007, 9, 1291-1294. 